\title{
CANONICAL MEASURES AND KÄHLER-RICCI FLOW
}

\author{
JIAN SONG AND GANG TIAN
}

\section{Contents}

1. Introduction

2. Preliminaries

2.1. Kodaira dimension and semi-ample fibrations 308

\begin{tabular}{ll|}
2.2. & Iitaka fibrations \\
\hline
\end{tabular}

2.3. Analytic Zariski decomposition $\quad 310$

2.4. Complex Monge-Ampère equations $\quad 310$

3. Canonical metrics for semi-ample canonical bundle 311

3.1. Canonical metrics on canonical models 311

3.2. Existence and uniqueness 317

4. Canonical measures on projective manifolds of nonnegative Kodaira dimension

4.1. Canonical measures on surfaces of nonnegative Kodaira dimension

4.2. Ricci-flat metrics on Kähler manifolds of zero Kodaira dimension

4.3. Kähler-Einstein metrics on projective manifolds of general type

4.4. Projective manifolds of positive Kodaira dimension

4.5. Uniqueness assuming finite generation of the canonical ring

5. The Kähler-Ricci flow

5.1. Reduction of the normalized Kähler-Ricci flow

5.2. Kähler-Ricci flow on projective manifolds with semi-positive canonical line bundle

5.3. Kähler-Ricci flow and minimal model program

6. Adjunction formulas for energy functionals

6.1. Generalized constant scalar curvature Kähler metrics

6.2. Asymptotics of the Mabuchi energy by the large Kähler structure limits

Acknowledgments

References

Received by the editors November 25, 2008 and, in revised form, August 7, 2010.

2010 Mathematics Subject Classification. Primary 53-XX; Secondary 14-XX.

Key words and phrases. Ricci flow, Kähler-Einstein metrics, complex Monge-Ampère equations.

This research is supported in part by National Science Foundation grants DMS-0604805 and DMS-0804095. 


\section{INTRODUCTION}

It has been the subject of intensive study over the last few decades to study the existence of Kähler-Einstein metrics on a compact Kähler manifold, following Yau's solution to the Calabi conjecture (cf. [Ya2, [Au, Ti2, Ti3]). The Ricci flow (cf. [Ha, Ch] ) provides a canonical deformation of Kähler metrics in Kähler geometry. Cao $\mathrm{Ca}$ gave an alternative proof of the existence of Kähler-Einstein metrics on a compact Kähler manifold with trivial or negative first Chern class by the Kähler-Ricci flow. However, most projective manifolds do not have a definite or trivial first Chern class. It is a natural question to ask if there exist any well-defined canonical metrics on these manifolds or on varieties canonically associated to them. Tsuji [Ts1] applied the Kähler-Ricci flow and proved the existence of a canonical singular Kähler-Einstein metric on a minimal projective manifold of general type. It was the first attempt to relate the Kähler-Ricci flow and canonical metrics to the minimal model program. Since then, many interesting results have been achieved in this direction. The long time existence of the Kähler-Ricci flow on a minimal projective manifold with any initial Kähler metric is established in TiZha. The regularity problem of the canonical singular Kähler-Einstein metrics on minimal projective manifolds of general type is intensively studied in [Zh, EyGuZe1.

In this paper, we propose a program of finding canonical measures on projective varieties of positive Kodaira dimension. Such a canonical measure can be considered as a birational invariant and it induces a canonical singular metric on the canonical model, generalizing the notion of Kähler-Einstein metrics.

Let $X$ be an $n$-dimensional compact Kähler manifold. A Kähler metric can be given by its Kähler form $\omega$ on $X$. In local coordinates $z_{1}, \ldots, z_{n}$, we can write $\omega$ as

$$
\omega=\sqrt{-1} \sum_{i, j=1}^{n} g_{i \bar{j}} d z_{i} \wedge d z_{\bar{j}},
$$

where $\left\{g_{i \bar{j}}\right\}$ is a positive definite Hermitian matrix function. Consider the normalized Kähler-Ricci flow

$$
\left\{\begin{array}{l}
\frac{\partial \omega(t, \cdot)}{\partial t}=-\operatorname{Ric}(\omega(t, \cdot))-\omega(t, \cdot), \\
\omega(0, \cdot)=\omega_{0},
\end{array}\right.
$$

where $\omega(t, \cdot)$ is a family of Kähler metrics on $X, \operatorname{Ric}(\omega(t, \cdot))$ denotes the Ricci curvature of $\omega(t, \cdot)$ and $\omega_{0}$ is a given Kähler metric.

Let $X$ be a minimal projective manifold. If the canonical line bundle $K_{X}$ of $X$ is ample and $\omega_{0}$ represents $\left[K_{X}\right]$, it is proved in [Ca that (1.1) has a global solution $\omega(t, \cdot)$ for all $t \geq 0$ and $\omega(t, \cdot)$ converges to a unique Kähler-Einstein metric on $X$. Tsuji showed in [Ts1] that (1.1) has a global solution $\omega(t, \cdot)$ under the assumption that $K_{X}$ is nef and the initial Kähler class $\left[\omega_{0}\right]>\left[K_{X}\right]$. This additional assumption was removed in [TiZha; moreover, if $K_{X}$ is nef and big, $\omega(t, \cdot)$ converges to a singular Kähler-Einstein metric with locally bounded Kähler potential as $t$ tends to $\infty$ (see Ts1, TiZha $)$.

If $K_{X}$ is not big, the Kodaira dimension of $X$ is smaller than its complex dimension. In particular, when $X$ is a minimal Kähler surface of Kodaira dimension 1, it must be a minimal elliptic surface and does not admit any Kähler-Einstein current in $-2 \pi c_{1}(X)$, with bounded local potential smooth outside a subvariety. Hence, one 
does not expect that $\omega(t, \cdot)$ converges to a smooth Kähler-Einstein metric outside a subvariety of $X$ in general.

Let $f: X \rightarrow X_{\text {can }}$ be a minimal elliptic surface of $\operatorname{kod}(X)=1$. Suppose all the singular fibres are given by $X_{s_{1}}=f^{-1}\left(s_{1}\right), \ldots, X_{s_{k}}=f^{-1}\left(s_{k}\right)$ of multiplicity $m_{i} \in \mathbf{N}, i=1, \ldots, k$. In SoTi], the authors proved that the Kähler-Ricci flow on $X$ converges for any initial Kähler metric to a positive current $\omega_{\text {can }}$ on the canonical model $X_{c a n}$ satisfying

$$
\operatorname{Ric}\left(\omega_{c a n}\right)=-\omega_{c a n}+\omega_{W P}+2 \pi \sum_{i=1}^{k} \frac{m_{i}-1}{m_{i}}\left[s_{i}\right],
$$

where $\omega_{W P}$ is the induced Weil-Petersson metric and $\left[s_{i}\right]$ is the current of integration associated to the divisor $s_{i}$ on $X_{c a n}$. $\omega_{c a n}$ is called a generalized KählerEinstein metric on $X_{\text {can }}$. Moreover, the Kähler-Ricci flow is collapsing onto $X_{\text {can }}$ exponentially fast with uniformly bounded scalar curvature away from the singular fibres.

The first result of this paper is to generalize the above convergence result on the Kähler-Ricci flow to projective manifolds of positive Kodaira dimension and semi-ample canonical bundle.

Let $X$ be an $n$-dimensional projective manifold of Kodaira dimension $0<\kappa<n$. We assume that the canonical line bundle $K_{X}$ is semi-ample. Then the canonical ring $R\left(X, K_{X}\right)$ is finitely generated and the pluricanonical system induces an algebraic fibre space $f: X \rightarrow X_{\text {can }}$. We denote by $X_{\text {can }}^{\circ}$ the set of all nonsingular points $s \in X_{\text {can }}$ such that $f$ is nondegenerate at each point in $f^{-1}(s)$. Each nonsingular fibre of $f$ is a nonsingular Calabi-Yau manifold of dimension $n-\kappa$. We further let $X^{\circ}=f^{-1}\left(X_{c a n}^{\circ}\right)$. The $L^{2}$-metric on the moduli space of nonsingular Calabi-Yau manifolds induces a semi-positive $(1,1)$-form $\omega_{W P}$ of Weil-Petersson type on $X_{c a n}^{\circ}$. We will study the Kähler-Ricci flow starting from any Kähler metric and describe its limiting behavior as time goes to infinity.

Theorem A. Let $X$ be a nonsingular projective variety with semi-ample canonical line bundle $K_{X}$ and so $X$ admits an algebraic fibration $f: X \rightarrow X_{\text {can }}$ over its canonical model $X_{\text {can }}$. Suppose $0<\operatorname{dim} X_{\text {can }}=\kappa<\operatorname{dim} X=n$. Then for any initial Kähler metric, the Kähler-Ricci flow (1.1) has a global solution $\omega(t, \cdot)$ for all time $t \in[0, \infty)$ satisfying:

(1) $\omega(t, \cdot)$ converges to $f^{*} \omega_{\text {can }} \in-2 \pi c_{1}(X)$ as currents for a positive closed $(1,1)$-current $\omega_{\text {can }}$ on $X_{\text {can }}$ with continuous local potential.

(2) $\omega_{\text {can }}$ is smooth on $X_{\text {can }}^{\circ}$ and satisfies the generalized Kähler-Einstein equation on $X_{c a n}^{\circ}$,

(3) For any compact subset $K \in X_{\text {can }}^{\circ}$, there is a constant $C_{K}$ such that

$$
\|R(t, \cdot)\|_{L^{\infty}\left(f^{-1}(K)\right)}+\left.e^{(n-\kappa) t} \sup _{s \in K}|| \omega^{n-\kappa}(t, \cdot)\right|_{X_{s}} \|_{L^{\infty}\left(X_{s}\right)} \leq C_{K},
$$

where $X_{s}=f^{-1}(s)$.

Therefore, the Kähler-Ricci flow collapses onto the canonical model with bounded scalar curvature away from the singular fibres and the volume of each nonsingular fibre tends to 0 exponentially fast. In fact, the local potential of $\omega(t, \cdot)$ converges on $X^{\circ}$ locally in the $C^{0}$-topology (cf. Proposition [5.4). It should also converge 
locally in the $C^{1,1}$-topology on $X^{\circ}$ as in the surface case (cf. SoTi]) and this will be studied in detail in a forthcoming paper.

Similar phenomena also appear in the real setting as a special type-III Ricci flow solution without the presence of singular fibres. It is discovered and intensively studied in [Lo].

The abundance conjecture in algebraic geometry predicts that the canonical line bundle is semi-ample if it is nef. If the abundance conjecture is true, then Theorem A immediately implies that on all nonsingular minimal models of positive Kodaira dimension the Kähler-Ricci flow converges to a unique canonical metric on their canonical model.

In general, the canonical line bundle of a projective manifold of positive Kodaira dimension is not necessarily semi-ample or even nef. The minimal model program in birational geometry deals with the classification of projective varieties and aims to choose a minimal model in each birational equivalence class. Tsuji claimed in [Ts3] that there exists a singular Kähler-Einstein metric of analytic Zariski decomposition on projective manifolds of general type without assuming the finite generation of the canonical ring. Such a metric is constructed through a family of Kähler-Einstein metrics as the limits of a parabolic Monge-Ampère equation of Dirichlet type. The approach is interesting but rather complicated. The recent exciting development in the study of degenerate complex Monge-Ampère equations (cf. Kol1, Zh, EyGuZe1) enables the authors to give an independent and correct proof.

Theorem B1. Let $X$ be a projective manifold of general type. Then there exists a measure $\Omega_{K E}$ on $X$ such that

(1) $\left(K_{X}, \Omega_{K E}^{-1}\right)$ is an analytic Zariski decomposition.

(2) Let $\omega_{K E}=\sqrt{-1} \partial \bar{\partial} \log \Omega_{K E}$ be the closed positive $(1,1)$ current on $X$. Then there exists a nonempty Zariski open subset $U$ of $X$ such that $\operatorname{Ric}\left(\omega_{K E}\right)=$ $-\sqrt{-1} \partial \bar{\partial} \log \left(\omega_{K E}\right)^{n}$ is well defined on $U$ and

$$
\operatorname{Ric}\left(\omega_{K E}\right)=-\omega_{K E} .
$$

We remark that $\Omega_{K E} \leq C \Omega$ for a fixed smooth volume form on $X$ and a constant $C>0$. The proof of Theorem B.1 is given in Section 4.3. The existence of such a canonical Kähler-Einstein metric is also considered by Siu in Si2 as an alternative approach to attack the problem of the finite generation of canonical rings. A degenerate Monge-Ampère equation of Dirichlet type is considered, and the solution is expected to be unique. Indeed, if the canonical rings are finitely generated, such a solution coincides with the Kähler-Einstein metrics constructed in Theorem B.1. We hope that Theorem B.1 might help us to gain more understanding of the finite generation of canonical rings from an analytic point of view. Theorem B.1 can be generalized to projective manifolds of positive Kodaira dimension.

Theorem B2. Let $X$ be an n-dimensional projective manifold of Kodaira dimension $0<\kappa<n$. There exists a measure $\Omega_{\text {can }}$ on $X$ bounded above such that $\left(K_{X}, \Omega_{c a n}^{-1}\right)$ is an analytic Zariski decomposition. Let $\Phi^{\dagger}: X^{\dagger} \rightarrow Y^{\dagger}$ be any Iitaka fibration of $X$ with $\pi^{\dagger}: X^{\dagger} \rightarrow X$ and $\Omega^{\dagger}=\left(\pi^{\dagger}\right)^{*} \Omega_{\text {can }}$. Then:

(1) $\left(K_{X^{\dagger}},\left(\Omega^{\dagger}\right)^{-1}\right)$ is an analytic Zariski decomposition. 
(2) There exists a closed positive $(1,1)$-current $\omega^{\dagger}$ on $Y^{\dagger}$ such that $\left(\Phi^{\dagger}\right)^{*} \omega^{\dagger}=$ $\sqrt{-1} \partial \bar{\partial} \log \Omega^{\dagger}$ on a Zariski open set of $X^{\dagger}$. Furthermore, we have

$$
\begin{array}{r}
\left(\omega^{\dagger}\right)^{\kappa}=\left(\Phi^{\dagger}\right)_{*} \Omega^{\dagger} \\
\text { on } Y^{\dagger} \text { and so on a Zariski open set of } Y^{\dagger}, \\
\operatorname{Ric}\left(\omega^{\dagger}\right)=-\omega^{\dagger}+\omega_{W P} .
\end{array}
$$

The definition of $\omega_{W P}$ in Theorem B.2 is given in Section 4.4 and it is a generalization of the Weil-Petersson form induced from an algebraic deformation space of projective manifolds of Kodaira dimension 0 .

In fact, the Hermitian metric $\Omega_{c a n}^{-1}$ (also $\Omega_{K E}^{-1}$ ) on $K_{X}$ constructed in the proof of Theorem B.2 (also Theorem B.1) has stronger properties than the analytic Zariski decomposition. Let

$$
\Psi_{X, \epsilon}=\sum_{m=1}^{\infty} \sum_{j=0}^{d_{m}} \epsilon_{m, j}\left|\sigma_{m, j}\right|^{\frac{2}{m}},
$$

where $\left\{\sigma_{m, j}\right\}_{j=0}^{d_{m}}$ spans $H^{0}\left(X, m K_{X}\right)$ and $\left\{\epsilon_{m, j}>0\right\}$ is a sequence such that $\Psi_{X, \epsilon}$ is convergent. $\Psi_{X, \epsilon}$ is a measure or a semi-positive $(n, n)$-current on $X$, as defined in [Si1. Then from the construction of $\Omega_{c a n}$ in the proof of Theorem B.2 (also Theorem B.1)

$$
\frac{\Psi_{X, \epsilon}}{\Omega_{\text {can }}}<\infty .
$$

If the canonical ring $R\left(X, K_{X}\right)$ is finitely generated, one can replace $\Psi_{X, \epsilon}$ by

$$
\Psi_{X}=\sum_{m=0}^{M} \sum_{j=0}^{d_{m}}\left|\sigma_{m, j}\right|^{\frac{2}{m}}
$$

for some $M$ sufficiently large.

Recently, the finite generation of canonical rings on projective varieties of general type was proved independently by $\mathrm{BiCaHaMc}$ and $[\mathrm{Si3}]$. By assuming the finite generation of canonical rings, Theorems B.1 and B.2 can be strengthened and the proof can be very much simplified. It turns out that the canonical measure in Theorems B.1 and B.2 is unique and invariant under birational transformations.

Theorem C1. Let $X$ be a projective manifold of general type. If the canonical ring $R\left(X, K_{X}\right)$ is finitely generated, the Kähler-Einstein measure constructed in Theorem $B .1$ is continuous on $X$ and smooth on a Zariski open set of $X$. Furthermore, it is the pullback of the unique canonical Kähler-Einstein measure $\Omega_{K E}$ from $X_{\text {can }}$ satisfying

$$
\left(\sqrt{-1} \partial \bar{\partial} \log \Omega_{K E}\right)^{n}=\Omega_{K E} .
$$

The unique Kähler-Einstein metric with bounded local potential and the associated Kähler-Einstein measure on the canonical model $X_{\text {can }}$ are constructed in EyGuZe1. The Kähler-Einstein measure in Theorem C.1 is invariant under birational transformations and so it can be considered as a birational invariant. Theorem C.1 can also be generalized to all projective manifolds of positive Kodaira dimension. 
Theorem C2. Let $X$ be an $n$-dimensional projective manifold of Kodaira dimension $0<\kappa<n$. If the canonical ring $R\left(X, K_{X}\right)$ is finitely generated, then there exists a unique canonical measure $\Omega_{\text {can }}$ on $X$ satisfying:

(1) $0<\frac{\Psi_{X}}{\Omega_{c a n}}<\infty$.

(2) $\Omega_{\text {can }}$ is continuous on $X$ and smooth on a Zariski open set of $X$.

(3) Let $\Phi: X \rightarrow X_{\text {can }}$ be the pluricanonical map. Then there exists a unique closed positive $(1,1)$-current $\omega_{\text {can }}$ with bounded local potential on $X_{\text {can }}$ such that $\Phi^{*} \omega_{\text {can }}=\sqrt{-1} \partial \bar{\partial} \log \Omega_{\text {can }}$ outside the base locus of the pluricanonical system. Furthermore, on $X_{\text {can }}$,

$$
\left(\omega_{c a n}\right)^{\kappa}=\Phi_{*} \Omega_{c a n}
$$

and

$$
\operatorname{Ric}\left(\omega_{c a n}\right)=-\omega_{c a n}+\bar{\omega}_{W P} .
$$

In particular, $\Omega_{\text {can }}$ is invariant under birational transformations.

$\bar{\omega}_{W P}$ is defined in Section 4.5 (cf. Definition 4.2) and it coincides with $\omega_{W P}$ in Theorem B.2 on a Zariski open set of $X_{\text {can }}$ by choosing $Y^{\dagger}$ to be $X_{c a n}$. Theorem C.1 and Theorem 3.2 are proved in Section 4.5.

We now give a brief outline of the current paper. In Section 2, we present some well-known results in algebraic geometry and complex Monge-Ampère equations. In Section 3, we prove the existence and uniqueness for canonical metrics of Kähler-Einstein type on projective manifolds with semi-ample canonical bundle. In Section 4, we prove the existence of the canonical measures for projective manifolds of positive Kodaira dimension. In Section 5, we prove the convergence of the normalized Kähler-Ricci flow on projective manifolds with semi-ample canonical bundle. In Section 6 , we propose a generalized constant scalar curvature equation and also give an adjunction formula for the Mabuchi energy, which could be helpful for understanding the collapsing behavior of the normalized Kähler-Ricci flow.

\section{Preliminaries}

2.1. Kodaira dimension and semi-ample fibrations. Let $X$ be an $n$-dimensional compact complex projective manifold and $L \rightarrow X$ a holomorphic line bundle over $X$. Let $N(L)$ be the semi-group defined by

$$
N(L)=\left\{m \in \mathbf{N} \mid H^{0}\left(X, L^{m}\right) \neq 0\right\} .
$$

Given any $m \in N(L)$, the linear system $\left|L^{m}\right|=\mathbf{P} H^{0}\left(X, L^{m}\right)$ induces a rational $\operatorname{map} \Phi_{m}$

$$
\Phi_{m}: X \rightarrow \mathbf{C P}^{d_{m}}
$$

by any basis $\left\{\sigma_{m, 0}, \sigma_{m, 1}, \ldots, \sigma_{m, d_{m}}\right\}$ of $H^{0}\left(X, L^{m}\right)$,

$$
\Phi_{m}(z)=\left[\sigma_{m, 0}, \sigma_{m, 1}, \ldots, \sigma_{m, d_{m}}(z)\right],
$$

where $d_{m}+1=\operatorname{dim} H^{0}\left(X, L^{m}\right)$. Let $Y_{m}=\Phi_{m}(X) \subset \mathbf{C P}^{d_{m}}$ be the image of the closure of the graph of $\Phi_{m}$.

Definition 2.1. The Iitaka dimension of $L$ is defined to be

$$
\kappa(X, L)=\max _{m \in N(L)}\left\{\operatorname{dim} Y_{m}\right\}
$$

if $N(L) \neq \phi$, and $\kappa(X, L)=-\infty$ if $N(L)=\phi$. 
Definition 2.2. Let $X$ be a projective manifold and $K_{X}$ the canonical line bundle over $X$. Then the Kodaira dimension $\operatorname{kod}(X)$ of $X$ is defined to be

$$
\operatorname{kod}(X)=\kappa\left(X, K_{X}\right) .
$$

The Kodaira dimension is a birational invariant of a projective variety and the Kodaira dimension of a singular variety is equal to that of its smooth model.

Definition 2.3. Let $L \rightarrow X$ be a holomorphic line bundle over a compact projective manifold $X . L$ is called semi-ample if $L^{m}$ is globally generated for some $m>0$.

For any $m \in \mathbf{N}$ such that $L^{m}$ is globally generated, the linear system $\left|L^{m}\right|$ induces a holomorphic map $\Phi_{m}$,

$$
\Phi_{m}: X \rightarrow \mathbf{C P}^{d_{m}}
$$

by any basis of $H^{0}\left(X, L^{m}\right)$. Let $Y_{m}=\Phi_{m}(X)$, and so $\Phi_{m}$ can be considered as

$$
\Phi_{m}: X \rightarrow Y_{m} \text {. }
$$

The following theorem is well known (cf. [La, $\mathrm{Ue}]$ ).

Theorem 2.1. Let $L \rightarrow X$ be a semi-ample line bundle over a projective manifold $X$. Then there is an algebraic fibre space

$$
\Phi_{\infty}: X \rightarrow Y
$$

such that for any sufficiently large integer $m$ with $L^{m}$ being globally generated,

$$
Y_{m}=Y \text { and } \Phi_{m}=\Phi_{\infty},
$$

where $Y$ is a normal projective variety. Furthermore, there exists an ample line bundle $A$ on $Y$ such that $L^{m}=\left(\Phi_{\infty}\right)^{*} A$.

If $L$ is semi-ample, the graded ring $R(X, L)=\bigoplus_{m \geq 0} H^{0}\left(X, L^{m}\right)$ is finitely generated and so $R(X, L)$ is the coordinate ring of $Y$.

Definition 2.4. Let $L \rightarrow X$ be a semi-ample line bundle over a projective manifold $X$. Then the algebraic fibre space $\Phi_{\infty}: X \rightarrow Y$ as in Theorem 2.1 is called the Iitaka fibration associated to $L$ and it is completely determined by the linear system $\left|L^{m}\right|$ for sufficiently large $m$.

In particular, if the canonical bundle $K_{X}$ is semi-ample, the algebraic fibre space associated to $K_{X}$,

$$
f: X \rightarrow X_{\text {can }}
$$

is called the Iitaka fibration of $X$, where $f=\Phi_{\infty}$ and $X_{\text {can }}$ is called the canonical model of $X$.

2.2. Iitaka fibrations. In general, the canonical line bundle is not necessarily semi-ample, and the asymptotic behavior of the pluricanonical maps is characterized by the following fundamental theorem on Kodaira dimensions due to Iitaka (cf. [U] $)$.

Theorem 2.2. Let $X$ be an n-dimensional projective manifold of positive Kodaira dimension. Then for all sufficiently large $m \in N\left(K_{X}\right)$, the pluricanonical maps $\Phi_{m}: X \rightarrow Y_{m}$ are birationally equivalent to an algebraic fibre space

$$
\Phi^{\dagger}: X^{\dagger} \rightarrow Y^{\dagger}
$$


unique up to birational equivalence satisfying:

(1) There exists a commutative diagram for sufficiently large $m \in N\left(K_{X}\right)$

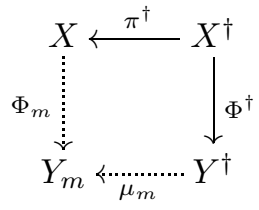

of rational maps with $\pi^{\dagger}$ and $\mu_{m}$ being birational.

(2) $\operatorname{dim} Y^{\dagger}=\operatorname{kod}(X)$.

(3) A very general fibre of $\Phi^{\dagger}$ has Kodaira dimension 0.

2.3. Analytic Zariski decomposition. Let $X$ be a compact complex manifold and $L$ be a holomorphic line bundle on $X$ equipped with a smooth Hermitian metric $h_{0}$.

A singular Hermitian metric $h$ on $L$ is given by

$$
h=h_{0} e^{-\varphi}
$$

for some $\varphi \in L^{1}(M)$.

Let $\Theta_{h_{0}}$ be the curvature of $h_{0}$ defined by

$$
\Theta_{h_{0}}=-\sqrt{-1} \partial \bar{\partial} \log h_{0} .
$$

Then the curvature $\Theta_{h}$ of $h$ as a current is defined by

$$
\Theta_{h}=\Theta_{h_{0}}+\sqrt{-1} \partial \bar{\partial} \varphi
$$

Definition 2.5. $L$ is called pseudoeffective if there exists a singular Hermitian metric $h$ on $L$ such that the curvature $\Theta_{h}$ is a closed positive current. Let

$$
\mathcal{P}_{h_{0}}(X)=\left\{\varphi \in L^{1}(X) \mid \Theta_{h_{0}}+\sqrt{-1} \partial \bar{\partial} \varphi \geq 0 \text { as current }\right\} .
$$

Definition 2.6. Let $\varphi \in \mathcal{P}_{h_{0}}(X)$ and $h=h_{0} e^{-\varphi}$. The multiplier ideal sheaf $\mathcal{I}(h) \subset \mathcal{O}_{X}(L)$ or $\mathcal{I}(\varphi)$ is defined by

$$
\Gamma(U, \mathcal{I}(h))=\left\{\left.f \in \Gamma\left(U, \mathcal{O}_{X}(L)\right)|| f\right|_{h_{0}} ^{2} e^{-\varphi} \in L_{l o c}^{1}(U)\right\} .
$$

The notion of analytic Zariski decomposition is an analytic analog of Zariski decomposition and it is introduced in Ts1] to study a pseudoeffective line bundle.

Definition 2.7. A singular Hermitian metric $h$ on $L$ is an analytic Zariski decomposition if

(1) $\Theta_{h}$ is a closed semi-positive current,

(2) for every $m \geq 0$, the natural inclusion

$$
H^{0}\left(X, \mathcal{O}_{X}(m L) \otimes \mathcal{I}\left(h^{m}\right)\right) \rightarrow H^{0}\left(X, \mathcal{O}_{X}(m L)\right)
$$

is an isomorphism.

2.4. Complex Monge-Ampère equations. Let $X$ be an $n$-dimensional Kähler manifold and let $\omega$ be a smooth closed semi-positive $(1,1)$-form. $\omega$ is Kähler if it is positive and $\omega$ is called big if $[\omega]^{n}=\int_{X} \omega^{n}>0$.

Definition 2.8. A quasi-plurisubharmonic function associated to $\omega$ is a function $\varphi: X \rightarrow[-\infty, \infty)$ such that for any smooth local potential $\psi$ of $\omega, \psi+\varphi$ is plurisubharmonic. We denote by $\operatorname{PSH}(X, \omega)$ the set of all quasi-plurisubharmonic functions associated to $\omega$ on $X$. 
The following comparison principle for quasi-plurisubhharmonic functions on compact Kähler manifolds is well known.

Theorem 2.3. Let $X$ be an n-dimensional Kähler manifold. Suppose $\varphi, \psi \in$ $P S H(X, \omega) \cap L^{\infty}(X)$ for a big smooth closed semi-positive $(1,1)$-form $\omega$. Then

$$
\int_{\varphi<\psi}(\omega+\sqrt{-1} \partial \bar{\partial} \psi)^{n} \leq \int_{\varphi<\psi}(\omega+\sqrt{-1} \partial \bar{\partial} \phi)^{n} .
$$

In Kol1, Kolodziej proved the fundamental theorem on the existence of continuous solutions to the Monge-Ampère equation $(\omega+\sqrt{-1} \partial \bar{\partial} \varphi)^{n}=F \omega^{n}$, where $\omega$ is a Kähler form and $F \in L^{p}\left(X, \omega^{n}\right)$ for some $p>1$. Its generalization was independently carried out in $[\mathrm{Zh}]$ and EyGuZe1]. They proved that there is a bounded solution when $\omega$ is semi-positive and big. These generalizations are summarized in the following.

Theorem 2.4. Let $X$ be an n-dimensional Kähler manifold and let $\omega$ be a big smooth closed semi-positive (1,1)-form. Then there exists a unique solution $\varphi \in$ $P S H(X, \omega) \cap L^{\infty}(X)$ to the following Monge-Ampère equation:

$$
(\omega+\sqrt{-1} \partial \bar{\partial} \varphi)^{n}=F \Omega,
$$

where $\Omega>0$ is a smooth volume form on $X, F \in L^{p}(X, \Omega)$ for some $p>1$ and $\int_{X} F \Omega=\int_{X} \omega^{n}$.

The continuity of the solution is given in [DiZh (also see [Zh for an earlier and sketched proof) if $X$ is projective and $\omega$ lies in the class of a semi-ample line bundle. Recently, Demailly and Pali proved the following uniform estimate and we refer the readers to the general statement in $\mathrm{DePa}$. Such an $L^{\infty}$-estimate is also independently obtained in EyGuZe2.

Theorem 2.5. Let $X$ be an $n$-dimensional Kähler manifold. Let $\Omega>0$ be a smooth volume form and $\omega$ a Kähler form on $X$. Suppose $\pi: X \rightarrow Y$ is a holomorphic map from $X$ to a Kähler manifold $Y$ and $\chi$ is the pullback of a Kähler form on $Y$. Let $\omega_{t}=\chi+t \omega$ for $t \in(0,1)$. Let $\varphi_{t} \in P S H\left(X, \omega_{t}\right) \cap L^{\infty}(X)$ be a solution of the degenerate complex Monge-Ampère equation $\left(\omega_{t}+\sqrt{-1} \partial \bar{\partial} \varphi_{t}\right)^{n}=F_{t} \Omega$ with $F_{t} \in L^{p}(X)$ for some $p>1$. Suppose

$$
\int_{X}\left(\frac{F_{t}}{\left[\omega_{t}\right]^{n}}\right)^{p} \Omega \leq A .
$$

Then for $t \in(0,1]$,

$$
\sup _{X} \varphi_{t}-\inf _{X} \varphi_{t} \leq C\left(\Omega, \omega_{0}, \epsilon, p, A, B\right) .
$$

The estimate in Theorem 2.5 assumes very weak dependence on the reference form $\omega$ and it is essential in deriving the $C^{0}$-estimate for the Kähler-Ricci flow on projective manifolds with positive Kodaira dimension and semi-ample canonical line bundle (cf. Section 5.2).

\section{CAnonical metrics for Semi-Ample CANonical Bundle}

3.1. Canonical metrics on canonical models. Let $X$ be an $n$-dimensional complex projective manifold with semi-ample canonical line bundle $K_{X}$. Fix $m \in$ 
$N\left(K_{X}\right)$ sufficiently large and let $f=\Phi_{m}$. The Iitaka fibration of $X$ is then given by the following holomorphic map:

$$
f: X \rightarrow X_{\text {can }} \subset \mathbf{C P}^{d_{m}} .
$$

We assume that $0<\kappa=\operatorname{kod}(X)<n$, and so $X$ is an algebraic fibre space over $X_{\text {can }}$. Let

$X_{c a n}^{\circ}=\left\{y \in X_{c a n} \mid y\right.$ is a nonsingular fibre and $X_{y}=f^{-1}(s)$ is a nonsingular fibre $\}$ and $X^{\circ}=f^{-1}\left(X_{c a n}^{\circ}\right)$. The following proposition is well known.

Proposition 3.1. We have

$$
K_{X}=\frac{1}{m} f^{*} \mathcal{O}(1)
$$

For all $y \in X_{\text {can }}^{\circ}, K_{X_{y}}$ is numerically trivial and so $c_{1}\left(X_{y}\right)=0$.

Thus $X$ can be considered as a holomorphic fibration of polarized Calabi-Yau manifolds over its canonical model $X_{c a n}$. Since $f: X \rightarrow X_{c a n} \in \mathbf{C P}^{d_{m}}$ and $-c_{1}(X)=\frac{1}{m}\left[f^{*} \mathcal{O}(1)\right]$, we can define

$$
\chi=\frac{1}{m} \sqrt{-1} \partial \bar{\partial} \log \sum_{j=0}^{d_{m}}\left|\sigma_{m, j}\right|^{2} \in-2 \pi c_{1}(X)
$$

as a multiple of the pulled back Fubini-Study metric on $\mathbf{C P}^{d_{m}}$ by a basis $\left\{\sigma_{m, j}\right\}_{j=0}^{d_{m}}$ $\subset H^{0}\left(X, K_{X}^{m}\right)$.

We can also consider $\chi$ as the restriction of the multiple of the Fubini-Study metric on the normal variety $X_{c a n}$ and we identify $\chi$ and $f^{*} \chi$ for convenience.

Let $\Omega=\sum_{j=0}^{d_{m}}\left|\sigma_{m, j}\right|^{2}$. Since $K_{X}^{m}$ is base point free, $\Omega$ is a smooth nondegenerate volume form on $X$ such that

$$
\sqrt{-1} \partial \bar{\partial} \log \Omega=\chi
$$

Definition 3.1. The pushforward $f_{*} \Omega$ with respect to the holomorphic map $f$ : $X \rightarrow X_{c a n}$ is defined as currents as the following. For any continuous function $\psi$ on $X_{c a n}$, we then immediately have the following lemma:

$$
\int_{X_{c a n}} \psi f_{*} \Omega=\int_{X}\left(f^{*} \psi\right) \Omega
$$

For any continuous function $\psi$ on $X_{c a n}$,

$$
\int_{X_{c a n}} \psi f_{*} \Omega=\int_{X}\left(f^{*} \psi\right) \Omega=\int_{y \in X_{c a n}} \int_{X_{y}}\left(f^{*} \psi\right) \Omega .
$$

Then we immediately have the following lemma.

Lemma 3.1. On $X_{\text {can }}^{\circ}$,

$$
f_{*} \Omega=\int_{X_{y}} \Omega
$$

Definition 3.2. We define a function $F$ on $X_{c a n}$ by

$$
F=\frac{f_{*} \Omega}{\chi^{\kappa}} .
$$


Lemma 3.2. Given any Kähler class $[\omega]$ on $X$, there is a smooth function $\psi$ on $X^{\circ}$ such that $\omega_{S F}:=\omega+\sqrt{-1} \partial \bar{\partial} \psi$ is a closed semi-flat (1,1)-form in the following sense: the restriction of $\omega_{S F}$ to each smooth $X_{y} \subset X^{\circ}$ is a Ricci flat Kähler metric.

Proof. For each $y \in X_{c a n}^{\circ}$, let $\omega_{y}$ be the restriction of $\omega$ to $X_{y}$ and $\partial_{V}$ and $\bar{\partial}_{V}$ be the restriction of $\partial$ and $\bar{\partial}$ to $X_{y}$. Then by the Hodge theory, there is a unique function $h_{y}$ on $X_{y}$ defined by

$$
\left\{\begin{array}{l}
\partial_{V} \bar{\partial}_{V} h_{y}=-\partial_{V} \bar{\partial}_{V} \log \omega_{y}^{n-\kappa}, \\
\int_{X_{y}} e^{h_{y}} \omega_{y}^{n-\kappa}=\int_{X_{y}} \omega_{y}^{n-\kappa} .
\end{array}\right.
$$

By Yau's solution to the Calabi conjecture, there is a unique $\psi_{y}$ solving the following Monge-Ampère equation:

$$
\left\{\begin{array}{l}
\frac{\left(\omega_{y}+\sqrt{-1} \partial_{V} \bar{\partial}_{V} \psi_{y}\right)^{n-\kappa}}{\omega_{y}^{n-\kappa}}=e^{h_{y}}, \\
\int_{X_{y}} \psi_{y} \omega_{y}^{n-\kappa}=0 .
\end{array}\right.
$$

Since $f$ is holomorphic, $\psi(z, y)=\psi_{y}(z)$ is well defined as a smooth function on $X^{\circ}$.

For each $y \in X_{c a n}^{\circ}$, there exists a holomorphic $(n-\kappa, 0)$-form $\eta$ on $X_{y}$ such that $\eta \wedge \bar{\eta}$ is a Calabi-Yau volume form and $\int_{X_{y}} \eta \wedge \bar{\eta}=\int_{X_{y}}\left(\left.\omega\right|_{X_{y}}\right)^{n-\kappa}$.

Definition 3.3. The closed $(n-\kappa, n-\kappa)$-current $\Theta$ on $X^{\circ}$ is defined to be

$$
\Theta=\left(\omega_{S F}\right)^{n-\kappa} \text {. }
$$

Let $\Theta_{y}=\left.\Theta\right|_{X_{y}}$ for $y \in X_{c a n}^{\circ}$ be the restriction of $\Theta$ on a nonsingular fibre $X_{y}$. Then $\Theta_{y}$ is a smooth Calabi-Yau volume form with

$$
\int_{X_{y}} \Theta_{y}=[\omega]^{n-\kappa} \cdot X_{y}=\text { constant. }
$$

We can always scale $[\omega]$ such that $[\omega]^{n-\kappa} \cdot X_{y}=1$ for $y \in X_{c a n}^{\circ}$.

Lemma 3.3. On $X^{\circ}$, we have

$$
f^{*} F=\left(\frac{\Omega}{\Theta \wedge \chi^{\kappa}}\right) .
$$

Furthermore, $\Theta$ can be extended to $X$ as a current such that $f^{*} F=\left(\frac{\Omega}{\Theta \wedge \chi^{\kappa}}\right)$ on $X$.

Proof. Let $\mathcal{F}=\left(\frac{\Omega}{\Theta \wedge \chi^{\kappa}}\right)$ be defined on $X^{\circ}$. We first show that $\mathcal{F}$ is constant along each fibre $X_{y}$ for $y \in X_{c a n}^{\circ}$.

Since $\chi$ is the pullback from $X_{\text {can }}$, we have

$$
\sqrt{-1} \partial_{V} \bar{\partial}_{V} \log \Omega=\sqrt{-1} \partial_{V} \bar{\partial}_{V} \log \Theta \wedge \chi^{\kappa}=0
$$

on each nonsingular fibre $X_{y}$. On the other hand, $\mathcal{F}$ is smooth on each $X_{y}$ for $y \in X_{\text {can }}^{\circ}$; therefore $\mathcal{F}$ is constant along $X_{y}$ and $\mathcal{F}$ can be considered as the pullback of a function from $X_{c a n}^{\circ}$. 
Now we can show (3.15). Let $\psi$ be any smooth test function on $X_{\text {can }}^{\circ}$. Let $y_{0}$ be a fixed point in $X_{c a n}^{\circ}$. Then

$$
\int_{X_{c a n}^{\circ}} \psi F \chi^{\kappa}=\int_{X_{c a n}^{\circ}} \psi f_{*} \Omega=\int_{y \in X_{c a n}^{\circ}} \psi\left(\int_{X_{y}} \Omega\right)=\int_{X^{\circ}} \psi \Omega .
$$

On the other hand,

$$
\begin{aligned}
\int_{X_{c a n}^{\circ}} \psi \mathcal{F} \chi^{\kappa} & =\int_{y \in X_{c a n}^{\circ}} \psi\left(\frac{\Omega}{\Theta \wedge \chi^{\kappa}}\right)\left(\int_{X_{y}} \Theta_{y}\right) \chi^{\kappa} \\
& =\int_{y \in X_{c a n}^{\circ}} \int_{X_{y}} \psi\left(\frac{\Omega}{\Theta \wedge \chi^{\kappa}}\right) \Theta_{y} \wedge \chi^{\kappa} \\
& =\int_{X^{\circ}} \psi \Omega .
\end{aligned}
$$

Therefore $f^{*} F=\mathcal{F}$.

Let $\psi^{\prime}$ be any smooth test function on $X$. $\Theta$ can be extended as a current to $X$ such that

$$
\int_{X} \psi^{\prime} f^{*} F \Theta \wedge \chi^{\kappa}=\int_{X} \psi^{\prime} \Omega
$$

Proposition 3.2. $F$ is smooth on $X_{\text {can }}^{\circ}$ and there exists $\epsilon>0$ such that

$$
F \in L^{1+\epsilon}\left(X_{c a n}\right) \text {. }
$$

Proof. Calculate

$$
\int_{X_{c a n}} F^{1+\epsilon} \chi^{\kappa}=\int_{X}\left(f^{*} F\right)^{1+\epsilon} \chi^{\kappa} \wedge \Theta=\int_{X}\left(f^{*} F\right)^{\epsilon} \Omega .
$$

Also for any $y \in X_{c a n}^{\circ}$, we can choose $z_{0} \in X_{y}$ such that on $X_{y}, \omega^{n-\kappa}\left(z_{0}\right)=$ $\omega_{S F}^{n-\kappa}\left(z_{0}\right)$ since $X_{y}$ is smooth and $\int_{X_{y}} \omega^{n-\kappa}=\int_{X_{y}} \omega_{S F}^{n-\kappa}$. Then

$$
\begin{aligned}
|F(y)| & =\frac{\Omega}{\chi^{\kappa} \wedge \omega_{S F}^{n-\kappa}} \\
& =\frac{\Omega}{\chi^{\kappa} \wedge \omega^{n-\kappa}} \frac{\omega^{n-\kappa}}{\omega_{S F}^{n-\kappa}}\left(z_{0}\right) \\
& =\frac{\Omega}{\chi^{\kappa} \wedge \omega^{n-\kappa}}\left(z_{0}\right) \\
& \leq \sup _{X_{y}} \frac{\Omega}{\chi^{\kappa} \wedge \omega^{n-\kappa}} .
\end{aligned}
$$

Therefore $F$ is bounded by poles and $\left(f^{*} F\right)^{\epsilon}$ is integrable for sufficiently small $\epsilon>0$.

Proposition 3.3. Let $\pi: Y \rightarrow X_{\text {can }}$ be a smooth model of $X_{\text {can }}$ by resolution of singularities of $X_{\text {can }} . \pi^{*} F$ has at worst pole singularities on $Y$.

Proof. Let $D$ be a divisor on $X_{\text {can }}$ such that $X_{\text {can }} \backslash X_{\text {can }}^{\circ} \subset S_{D}$. Let $S_{D}$ be the defining section of $D$ and $h_{D}$ be the Hermitian metric on the line bundle associated 
to $[D]$ such that $\pi^{*}\left(\left|S_{D}\right|_{h_{D}}^{2}\right)$ is a smooth function. For any continuous volume form $\Omega^{\prime}$ on $X$,

$$
\begin{aligned}
f_{*}\left(\left|S_{D}\right|_{h_{D}}^{2 N} \Omega^{\prime}\right) & \\
& =\int_{X_{s}}\left|S_{D}\right|_{h_{D}}^{2 N} \Omega^{\prime} \\
& =\left(\int_{X_{s}}\left|S_{D}\right|_{h_{D}}^{2 N}\left(\frac{\Omega^{\prime}}{\chi^{\kappa} \wedge \omega^{n-\kappa}}\right)\left(\left.\omega\right|_{X_{s}}\right)^{n-\kappa}\right) \chi^{\kappa}
\end{aligned}
$$

for $s \in X_{c a n}^{\circ}$. Since $\left|S_{D}\right|_{h_{D}}^{2 N} \frac{\Omega^{\prime}}{\chi^{\kappa} \wedge \omega^{n-\kappa}}<\infty$ for sufficiently large $N$, there exists a constant $C$ such that

$$
0 \leq f_{*}\left(\left|S_{D}\right|_{h_{D}}^{2 N} \Omega^{\prime}\right)<\chi^{\kappa} .
$$

Let $\mathcal{F}_{N}=\left|S_{D}\right|_{h_{D}}^{2 N} F$. Then on $X_{c a n}^{\circ}$,

$$
\begin{aligned}
\sqrt{-1} & \partial \bar{\partial} \mathcal{F}_{N} \\
& =\sqrt{-1} \partial \bar{\partial}\left(\int_{X_{s}}\left|S_{D}\right|_{h_{D}}^{2 N} \frac{\Omega}{\chi^{\kappa} \wedge \omega^{n-\kappa}} \omega^{n-\kappa}\right) \\
& =\int_{X_{s}} \sqrt{-1} \partial \bar{\partial}\left(\left|S_{D}\right|_{h_{D}}^{2 N} \frac{\Omega}{\chi^{\kappa} \wedge \omega^{n-\kappa}}\right) \wedge \omega^{n-\kappa} \\
& \leq C\left|S_{D}\right|_{h_{D}}^{2 M} \int_{X_{s}} \omega^{n-\kappa+1}
\end{aligned}
$$

for some sufficiently large $M$ by choosing $N$ sufficiently large.

Let $\eta$ be any semi-positive smooth $(\kappa-1, \kappa-1)$-form supported on $X_{c a n}^{\circ}$. Then

$$
\begin{aligned}
& \int_{X_{c a n}} \eta \wedge \sqrt{-1} \partial \bar{\partial} \mathcal{F}_{N} \\
& \leq C\left|S_{D}\right|_{h_{D}}^{2 M} \int_{X} f^{*} \eta \wedge \omega^{n-\kappa+1} \\
&= C\left|S_{D}\right|_{h_{D}}^{2 M} \int_{X_{c a n}}\left(\frac{\int_{X_{s}} \omega^{n-\kappa+1} \wedge \chi^{\kappa-1}}{\chi^{\kappa}}\right) f^{*} \eta \wedge \chi \\
& \leq C^{\prime}\left|S_{D}\right|_{h_{D}}^{2 L} \int_{X_{c a n}} \eta \wedge \chi
\end{aligned}
$$

if we choose $N$ sufficiently large.

A similar lower bound of $\sqrt{-1} \partial \bar{\partial} \mathcal{F}_{N}$ can be achieved and so for sufficiently large $N$, on $X_{\text {can }}^{\circ}$,

$$
-C \chi \leq \sqrt{-1} \partial \bar{\partial} \mathcal{F}_{N} \leq C \chi
$$

Let $\omega_{Y}$ be a Kähler metric on $Y$ and $\Delta_{Y}$ be the Laplace operator associated to $\omega_{Y}$. Then for sufficiently large $N$,

$$
\left|\Delta_{Y} \pi^{*} \mathcal{F}_{N}\right| \leq C
$$

Also we can assume that $\pi^{*}\left(\left|S_{D}\right|_{h_{D}}^{2 N}\right) \omega_{Y} \leq f^{*} \chi$ for sufficiently large $N$. After repeating the above estimates, we have for any $k \geq 0$, that there exists sufficiently large $N$ and $C_{k, N}$ such that

$$
\left|\left(\Delta_{Y}\right)^{k}\left(\pi^{*} \mathcal{F}_{N}\right)\right| \leq C_{k, N}
$$


By standard elliptic estimates, $\pi^{*} \mathcal{F}_{N}$ is uniformly bounded in $C^{k}$ if we choose $N$ sufficiently large; therefore $\pi^{*} F$ can have at worst pole singularities.

Now let us recall some facts on the Weil-Petersson metric on the moduli space $\mathcal{M}$ of polarized Calabi-Yau manifolds of dimension $n-\kappa$. Let $\mathcal{X} \rightarrow \mathcal{M}$ be a universal family of Calabi-Yau manifolds. Let $\left(U ; t_{1}, \ldots, t_{\kappa}\right)$ be a local holomorphic coordinate chart of $\mathcal{M}$, where $\kappa=\operatorname{dim} \mathcal{M}$. Then each $\frac{\partial}{\partial t_{i}}$ corresponds to an element $\iota\left(\frac{\partial}{\partial t_{i}}\right) \in H^{1}\left(\mathcal{X}_{t}, T_{\mathcal{X}_{t}}\right)$ through the Kodaira-Spencer map $\iota$. The Weil-Petersson metric is defined by the $L^{2}$-inner product of harmonic forms representing classes in $H^{1}\left(\mathcal{X}_{t}, T_{\mathcal{X}_{t}}\right)$. In the case of Calabi-Yau manifolds, as first shown in [Ti4], it can be expressed as follows. Let $\Psi$ be a nonzero holomorphic $(n-\kappa, 0)$-form on the fibre $\mathcal{X}_{t}$ and $\left.\Psi\right\lrcorner \iota\left(\frac{\partial}{\partial t_{i}}\right)$ be the contraction of $\Psi$ and $\frac{\partial}{\partial t_{i}}$. Then the Weil-Petersson metric is given by

$$
\left(\frac{\partial}{\partial t_{i}}, \frac{\partial}{\partial \bar{t}_{j}}\right)_{\omega_{W P}}=\frac{\left.\int_{\mathcal{X}_{t}} \Psi\right\lrcorner \iota\left(\frac{\partial}{\partial t_{i}}\right) \wedge \overline{\Psi\lrcorner \iota\left(\frac{\partial}{\partial t_{i}}\right)}}{\int_{\mathcal{X}_{t}} \Psi \wedge \bar{\Psi}} .
$$

One can also represent $\omega_{W P}$ as the curvature form of the first Hodge bundle $f_{*} \Omega_{\mathcal{X} / \mathcal{M}}^{n-\kappa}$ (cf. [Ti4]). Letting $\Psi$ be a nonzero local holomorphic section of $f_{*} \Omega_{\mathcal{X} / \mathcal{M}}^{n-\kappa}$, one can define the Hermitian metric $h_{W P}$ on $f_{*} \Omega_{\mathcal{X} / \mathcal{M}}^{n-\kappa}$ by

$$
\left|\Psi_{t}\right|_{h_{W P}}^{2}=\int_{\mathcal{X}_{t}} \Psi_{t} \wedge \overline{\Psi_{t}}
$$

Then the Weil-Petersson metric is given by

$$
\omega_{W P}=\operatorname{Ric}\left(h_{W P}\right) .
$$

The Weil-Petersson metric can also be considered as a canonical Hermitian metric on the dualizing sheaf $f_{*}\left(\Omega_{X / X_{\text {can }}}^{n-\kappa}\right)=\left(f_{* 1} \mathcal{O}_{X}\right)^{\vee}$ over $X_{\text {can }}^{\circ}$.

Let $X$ be an $n$-dimensional projective manifold. Suppose its canonical line bundle $K_{X}$ is semi-positive and $0<\kappa=\operatorname{kod}(X)<n$. Let $X_{\text {can }}$ be the canonical model of $X$. We define a canonical Hermitian metric $h_{\text {can }}$ on $f_{*}\left(\Omega_{X / X_{c a n}}^{n-\kappa}\right)$ in the way that for any smooth $(n-\kappa, 0)$-form $\eta$ on a nonsingular fibre $X_{y}$,

$$
|\eta|_{h_{W P}}^{2}=\frac{\eta \wedge \bar{\eta} \wedge \chi^{\kappa}}{\Theta \wedge \chi^{\kappa}}=\frac{\int_{X_{y}} \eta \wedge \bar{\eta}}{\int_{X_{y}} \Theta} .
$$

Definition 3.4. Let $X$ be an $n$-dimensional projective manifold with semi-ample canonical line bundle $K_{X}$. Suppose $0<\operatorname{kod}(X)<n$ and so $f: X \rightarrow X_{\text {can }}$ is a holomorphic fibration of Calabi-Yau manifolds. A closed positive $(1,1)$-current $\omega$ on $X_{\text {can }}$ is called a canonical metric if it satisfies the following:

(1) $f^{*} \omega \in-2 \pi c_{1}(X)$.

(2) $\omega$ is smooth outside a subvariety of $X_{c a n}$ and $\left(f^{*} \omega\right)^{\kappa} \wedge \Theta$ is continuous on $X$.

(3) $\operatorname{Ric}(\omega)=-\sqrt{-1} \partial \bar{\partial} \log \omega^{\kappa}$ is well defined on $X$ as a current and on $X_{\text {can }}^{\circ}$,

$$
\operatorname{Ric}(\omega)=-\omega+\omega_{W P} .
$$

Definition 3.5. Suppose $\omega_{c a n}$ is a canonical metric on $X_{c a n}$. We define the canonical volume form $\Omega_{\text {can }}$ on $X$ to be

$$
\Omega_{c a n}=\left(f^{*} \omega_{c a n}\right)^{\kappa} \wedge \Theta .
$$


3.2. Existence and uniqueness. The main goal of this section is to prove the existence and uniqueness for canonical metrics on the canonical models.

Theorem 3.1. Let $X$ be an $n$-dimensional projective manifold with semi-ample canonical line bundle $K_{X}$. Suppose $0<\kappa=\operatorname{kod}(X)<n$. Then there exists a unique canonical metric on $X_{\text {can }}$.

We will need the following theorem of solving the singular Monge-Ampère equation to prove Theorem 3.1 .

Theorem 3.2. There exists a unique solution $\varphi \in P S H(\chi) \cap C^{0}\left(X_{\text {can }}\right) \cap C^{\infty}\left(X_{\text {can }}^{\circ}\right)$ to the following Monge-Ampère equation on $X_{\text {can }}$ :

$$
(\chi+\sqrt{-1} \partial \bar{\partial} \varphi)^{\kappa}=F e^{\varphi} \chi^{\kappa} .
$$

Proof of Theorem 3.1. We will prove Theorem 3.1 by assuming Theorem 3.2 .

Let $\varphi$ be the solution in Theorem 3.1 and $\omega=\chi+\sqrt{-1} \partial \bar{\partial} \varphi$. Then

(1)

$$
f^{*} \omega=f^{*} \chi+\sqrt{-1} \partial \bar{\partial} f^{*} \varphi \in-2 \pi c_{1}(X),
$$

and it proves (1) in Definition 3.4

(2) By Theorem 3.2, $\omega$ is smooth on $X_{\text {can }}^{\circ}$ and

$$
\left(f^{*} \omega\right)^{\kappa} \wedge \Theta=\Omega e^{f^{*} \varphi}
$$

is continuous since $f^{*} \varphi$ is continuous on $X$ and $\Omega$ is a smooth volume form.

This proves (2) in Definition 3.4

(3) Then

$$
\operatorname{Ric}(\omega)=-\sqrt{-1} \partial \bar{\partial} \log \omega^{\kappa}=-\sqrt{-1} \partial \bar{\partial} \log \chi^{\kappa}-\sqrt{-1} \partial \bar{\partial} \log F-\sqrt{-1} \partial \bar{\partial} \varphi
$$

is well defined as a current on $X_{c a n}$.

Calculate on $X_{c a n}^{\circ}$ :

$$
\begin{aligned}
\sqrt{-1} \partial \bar{\partial} \log \chi^{\kappa}+\sqrt{-1} \partial \bar{\partial} \log F+\sqrt{-1} \partial \bar{\partial} \varphi \\
\quad=\sqrt{-1} \partial \bar{\partial} \log \chi^{\kappa}+\sqrt{-1} \partial \bar{\partial} \log \left(\frac{\Omega}{\Theta \wedge \chi^{\kappa}}\right)+\omega-\chi \\
\quad=\omega+\left(-\sqrt{-1} \partial \bar{\partial} \log \left(\Theta \wedge \chi^{\kappa}\right)+\sqrt{-1} \partial \bar{\partial} \log \chi^{\kappa}\right) \\
\quad=\omega-\omega_{W P} .
\end{aligned}
$$

Therefore

$$
\operatorname{Ric}(\omega)=-\omega+\omega_{W P} .
$$

So we have proved (3) in Definition 3.4.

Now we will prove the uniqueness of the canonical metric.

Let $\omega=\chi+\sqrt{-1} \partial \bar{\partial} \varphi$ be a canonical metric on $X_{\text {can }}$. Then by the equation for the canonical metric, we have on $X_{c a n}^{\circ}$,

$$
\sqrt{-1} \partial \bar{\partial} \log \left(\frac{\omega^{\kappa}}{\chi^{\kappa}}\right)=\sqrt{-1} \partial \bar{\partial} \log \left(\frac{\Omega}{\Theta \wedge \chi^{\kappa}} e^{\varphi}\right) .
$$

Let $\xi=\left(\frac{\omega^{\kappa}}{\chi^{\kappa}}\right)\left(\frac{\Omega}{\Theta \wedge \chi^{\kappa}} e^{\varphi}\right)^{-1}$. Then on $X_{\text {can }}^{\circ}$ we have

$$
\sqrt{-1} \partial \bar{\partial} \log \xi=0
$$


On the other hand,

$$
f^{*} \xi=\frac{\omega^{\kappa} \wedge \Theta}{\Omega} e^{-\varphi}
$$

extends to a strictly positive continuous function on $X$. Since $f$ is a holomorphic map, $\xi$ extends to a continuous function on $X_{\text {can }}$. Let $\pi: X_{c a n}^{\prime} \rightarrow X_{\text {can }}$ be a resolution of $X_{\text {can }}$. Then $\pi^{*} \xi$ is continuous on $X_{c a n}^{\prime}$ and $\sqrt{-1} \partial \bar{\partial} \log \pi^{*} \xi=0$ so that $\pi^{*} \xi=$ constant on $X_{c a n}^{\prime}$. Therefore $\xi=$ constant $>0$ on $X_{\text {can }}$ and $\varphi^{\prime}=\varphi+\log \xi \in P S H(\chi) \cap C^{0}\left(X_{c a n}\right)$ solves the Monge-Ampère equation (3.22). The uniqueness of the solution for (3.22) implies the uniqueness of the canonical metric.

Corollary 3.1. Let $\omega_{\text {can }}$ be a canonical metric on $X_{\text {can }}$ and $\Omega_{\text {can }}$ the canonical volume form on $X$. Then

$$
f^{*} \omega_{c a n}=\sqrt{-1} \partial \bar{\partial} \log \Omega_{c a n} .
$$

Proof. Let $\omega_{\text {can }}=\chi+\sqrt{-1} \partial \bar{\partial} \varphi$, where $\varphi$ is the solution of the Monge-Ampère equation (3.22) in Theorem 3.2, Then

$$
\Omega_{c a n}=\left(f^{*} \omega_{c a n}\right)^{\kappa} \wedge \Theta=\Omega e^{f^{*} \varphi}
$$

and so

$$
\sqrt{-1} \partial \bar{\partial} \log \Omega_{c a n}=\sqrt{-1} \partial \bar{\partial} \log \Omega+\sqrt{-1} \partial \bar{\partial} f^{*} \varphi=f^{*} \omega_{c a n}
$$

Proof of Theorem 3.2 .

\section{Step 1. Approximation.}

Let $\pi: Y \rightarrow X_{\text {can }}$ be a resolution of singularities such that $E=\pi^{*}\left(X_{\text {can }} \backslash X_{\text {can }}^{\circ}\right)$ is a divisor with simple normal crossings. Let $\hat{\chi}=\pi^{*} \chi$ and $\hat{F}=\pi^{*} F$. Then $\hat{\chi}$ is a closed semi-positive $(1,1)$-form on $Y$ and $\int_{Y} \hat{\chi}^{\kappa}=\int_{X_{c a n}} \chi^{\kappa}>0$, i.e., $\hat{\chi}$ is big. We will consider the following Monge-Ampère equation on $Y$ :

$$
\frac{(\hat{\chi}+\sqrt{-1} \partial \bar{\partial} \hat{\varphi})^{\kappa}}{\hat{\chi}^{\kappa}}=\hat{F} e^{\hat{\varphi}} .
$$

Since $\hat{\chi}$ is a big semi-positive closed $(1,1)$-form, so $\hat{L}=\frac{1}{m} \pi^{*} \mathcal{O}(1)$ is a semipositive big line bundle on $Y$. By Kodaira's lemma, there exists a divisor $D$ such that for any $\epsilon>0,[L]-\epsilon[D]$ is an ample divisor on $Y$. Let $S_{D}$ be the defining section of $D$ and choose a smooth Hermitian metric $h_{D}$ on the line bundle associated to $[D]$ such that

$$
\hat{\chi}+\epsilon \sqrt{-1} \partial \bar{\partial} \log h_{D}>0 .
$$

Fix $\epsilon_{0}>0$ and define a Kähler form $\omega_{0}=\hat{\chi}+\epsilon_{0} \sqrt{-1} \partial \bar{\partial} \log h_{D}>0 . \hat{F} \in$ $L^{1+\epsilon}\left(Y, \hat{\chi}^{\kappa}\right)$ for some $\epsilon>0$ since $F \in L^{1+\epsilon}\left(X_{c a n}, \chi^{\kappa}\right)$ for some $\epsilon>0$. Then for each $k>0$ there exists a family of functions $\left\{F_{j}\right\}_{j=1}^{\infty}$ on $Y$ satisfying the following.

(1) $F_{j} \in C^{4}(Y)$ for all $j$ and $F_{j} \rightarrow \hat{F}$ in $L^{1+\epsilon}\left(Y, \hat{\chi}^{\kappa}\right)$ as $j \rightarrow \infty$.

(2) There exists $C>0$ such that $\log F_{j} \geq-C$ for all $j$.

(3) There exist $\lambda, C>0$ such that for all $j$,

$$
\left\|\left|S_{E}\right|_{h_{E}}^{2 \lambda} F_{j}\right\|_{C^{2}(Y)} \leq C,
$$

where $S_{E}$ is a defining section of $E$ and $h_{E}$ is a fixed smooth Hermitian metric on the line bundle associated to $[E]$. 
For example, we can choose $\hat{F}_{j}$ to be defined by

$$
F_{j}=\exp \left(\left(\frac{\left|S_{E}\right|_{h_{E}}^{2 \alpha}}{j^{-1}+\left|S_{E}\right|_{h_{E}}^{2 \alpha}}\right) \log \hat{F}\right)
$$

for sufficiently large $\alpha>0$.

We let $\chi_{j}=\hat{\chi}+\frac{1}{j} \omega_{0}$. We consider the following Monge-Ampère equation:

$$
\frac{\left(\chi_{j}+\sqrt{-1} \partial \bar{\partial} \varphi_{j}\right)^{\kappa}}{\left(\chi_{j}\right)^{\kappa}}=F_{j} e^{\varphi_{j}}
$$

for sufficiently large $\alpha$.

By Yau's solution to the Calabi conjecture, for each $j$, there exists a unique solution $\varphi_{j} \in C^{3}(Y) \cap C^{\infty}(Y \backslash E)$ solving (3.25). We will derive uniform estimates for $\varphi_{j}$.

\section{Step 2. Zeroth-order estimates.}

Proposition 3.4. There exists $C>0$ such that for all $j$,

$$
\left\|\varphi_{j}\right\|_{L^{\infty}(Y)} \leq C \text {. }
$$

Furthermore, there exists a unique solution $\varphi_{\infty} \in P S H(Y, \hat{\chi}) \cap C^{0}(Y)$ solving the Monge-Ampère equation (3.24) such that

$$
\varphi_{j} \rightarrow \varphi_{\infty}
$$

in $L^{1}\left(Y, \omega_{0}^{\kappa}\right)$ as $j \rightarrow \infty$.

Proof. By Yau's theorem, for each $j$, there exists a smooth solution $\varphi_{j}$ solving the Monge-Ampère equation (3.24). We first derive a uniform upper bound for $\varphi_{j}$. Suppose that $\varphi_{j}$ achieves its maximum at $y_{0}$ on $Y$. Then applying the maximum principle, we have

$$
\varphi_{j} \leq \varphi_{j}\left(y_{0}\right) \leq \frac{1}{F_{j}\left(y_{0}\right)} \leq \sup _{Y}\left(\frac{1}{F_{j}}\right) \leq C .
$$

Let $\Omega_{0}$ be a smooth nowhere-vanishing volume form on $Y$. We have to verify that $F_{j}\left(\frac{\hat{\chi}_{j}^{\kappa}}{\Omega_{0}}\right)$ is uniformly bounded in $L^{1+\epsilon}\left(Y, \Omega_{0}\right)$ for some $\epsilon>0$ for all $j$.

$$
\begin{aligned}
\left\|e^{\varphi_{j}} F_{j}\left(\frac{\chi_{j}^{\kappa}}{\Omega_{0}}\right)\right\|_{L^{1+\epsilon}\left(Y, \Omega_{0}\right)}^{1+\epsilon} & =\int_{Y} e^{(1+\epsilon) \varphi_{j}} F_{j}^{1+\epsilon}\left(\frac{\chi_{j}^{\kappa}}{\Omega_{0}}\right)^{\epsilon} \chi_{j}^{\kappa} \\
& \geq C \int_{Y} F_{j}^{1+\epsilon} \chi_{j}^{\kappa}=C\left\|F_{j}\right\|_{L^{1+\epsilon}\left(Y, \Omega_{0}\right)}^{1+\epsilon}
\end{aligned}
$$

for some $C>0$. Therefore $\left\|\varphi_{j}\right\|_{L^{\infty}(Y)}$ is uniformly bounded by Theorem 2.4, since $\left\|F_{j}\right\|_{L^{1+\epsilon}\left(Y, \Omega_{0}\right)}^{1+\epsilon}$ is uniformly bounded.

Also Theorem 2.4 gives a unique solution $\varphi_{\infty} \in P S H(Y, \hat{\chi}) \cap C^{0}(Y)$ solving the Monge-Ampère equation (3.24). By the uniqueness of such a $\varphi_{\infty}$, we have $\varphi_{j} \rightarrow \varphi_{\infty}$ in $L^{1}$ by standard potential theory.

\section{Step 3. Second-order estimates.}

We now apply the maximum principle and prove a second-order estimate for $\varphi_{j}$ by using a modified argument in YYa2. Note that Tsuji used a similar trick in Ts1] for the second-order estimate. 
Let $\omega_{j}=\chi_{j}+\sqrt{-1} \partial \bar{\partial} \varphi_{j}, \Delta_{0}$ and $\Delta_{j}$ be the Laplace operators associated to $\omega_{0}$ and $\omega_{j}$. The following lemma is proved by a standard calculation.

Lemma 3.4. There exists a uniform constant $C>0$ only depending on $\omega_{0}$ such that on $Y \backslash(E \cup D)$,

$$
\Delta_{j} \log \operatorname{tr}_{\omega_{0}}\left(\omega_{j}\right) \geq-C\left(1+\operatorname{tr}_{\omega_{j}}\left(\omega_{0}\right)+\frac{1+\left|\Delta_{0} \log F_{j}\right|}{\operatorname{tr}_{\omega_{0}}\left(\omega_{j}\right)}\right) .
$$

Theorem 3.3. There exist $\alpha, \beta, C>0$ such that for all $j$ and $z \in Y \backslash(E \cup D)$,

$$
\operatorname{tr}_{\omega_{0}}\left(\omega_{j}\right)(z) \leq \frac{C}{\left|S_{E}\right|_{h_{E}}^{2 \alpha}\left|S_{D}\right|_{h_{D}}^{2 \beta}} .
$$

Proof. Define

and

$$
\Phi_{j}=\varphi_{j}-\epsilon_{0} \log \left|S_{D}\right|_{h_{D}}^{2}
$$

$$
H_{j}=\log \left(\left|S_{E}\right|_{h_{E}}^{2 \alpha} \operatorname{tr}_{\omega_{0}}\left(\omega_{j}\right)\right)-A \Phi_{j}
$$

for some constants $\alpha, A>0$ to be determined later.

First calculate on $Y \backslash(E \cup D)$ :

$$
\begin{aligned}
\Delta_{j} & H_{j} \\
& =\Delta_{j} \log \operatorname{tr}_{\omega_{0}}\left(\omega_{j}\right)-A \Delta_{j} \Phi_{j}+\alpha \Delta_{j} \log \left|S_{E}\right|_{h_{E}}^{2} \\
& =\Delta_{j} \log \operatorname{tr}_{\omega_{0}}\left(\omega_{j}\right)-A \operatorname{tr}_{\omega_{j}}\left(\omega_{j}-\omega_{0}\right)-\alpha \operatorname{tr}_{\omega_{j}}\left(\operatorname{Ric}\left(h_{E}\right)\right) \\
& \geq\left(A-C_{1}\right) \operatorname{tr}_{\omega_{j}}\left(\omega_{0}\right)-\alpha \operatorname{tr}_{\omega_{j}}\left(\operatorname{Ric}\left(h_{E}\right)\right)-\frac{C_{1}\left(1+\left|\Delta_{0} \log F_{j}\right|\right)}{\operatorname{tr}_{\omega_{0}}\left(\omega_{j}\right)}-\kappa A-C_{1} .
\end{aligned}
$$

Choose a sufficiently large $\alpha>0$, such that there exists a constant $C_{2}>0$ with the following:

$$
\frac{\left|S_{E}\right|_{h_{E}}^{2 \alpha}\left(1+\left|\Delta_{0} \log F_{j}\right|\right)}{\operatorname{tr}_{\omega_{0}}\left(\omega_{j}\right)} \leq \frac{C_{2}}{\operatorname{tr}_{\omega_{0}}\left(\omega_{j}\right)}
$$

and

$$
\left|S_{E}\right|_{h_{E}}^{2 \alpha} F_{j} \leq C_{2} .
$$

Apply the elementary inequality

$$
\begin{aligned}
\operatorname{tr}_{\omega_{j}}\left(\omega_{0}\right) & \geq C_{3}\left(\operatorname{tr}_{\omega_{0}}\left(\omega_{j}\right)\right)^{\frac{1}{\kappa-1}}\left(\frac{\omega_{0}^{\kappa}}{\omega_{j}^{\kappa}}\right)^{\frac{1}{\kappa-1}} \\
& =C_{3}\left(\frac{e^{-\varphi_{j}}}{F_{j}} \operatorname{tr}_{\omega_{0}}\left(\omega_{j}\right)\left(\frac{\omega_{0}^{\kappa}}{\chi_{j}^{\kappa}}\right)\right)^{\frac{1}{\kappa-1}} \\
& \geq C_{4}\left(\left|S_{E}\right|_{h_{E}}^{2 \alpha} \operatorname{tr}_{\omega_{0}}\left(\omega_{j}\right)\right)^{\frac{1}{\kappa-1}} .
\end{aligned}
$$

We can always choose $A$ sufficiently large such that

$$
\Delta_{j} H_{j} \geq C_{5} A\left(\left|S_{E}\right|_{h_{E}}^{2 \alpha} \operatorname{tr}_{\omega_{0}}\left(\omega_{j}\right)\right)^{\frac{1}{\kappa-1}}-\frac{C_{4}\left|S_{E}\right|_{h_{E}}^{4 \alpha}}{\left|S_{E}\right|_{h_{E}}^{2 \alpha} \operatorname{tr}_{\omega_{0}}\left(\omega_{j}\right)}-C_{6} A .
$$

For any $j$, suppose

$$
\sup _{z \in Y} H_{j}(z)=H_{j}\left(z_{0}\right)
$$


for some $z_{0} \in Y \backslash(E \cup D)$ since $H_{j}=-\infty$ along $E \cup D$. By the maximum principle, $\Delta_{j} H_{j}\left(z_{0}\right) \leq 0$. By a straightforward calculation, there exists $C_{7}>0$ such that

$$
\left.\left|S_{E}\right|_{h_{E}}^{2 \alpha} \operatorname{tr}_{\omega_{0}}\left(\omega_{j}\right)\right|_{z=z_{0}} \leq C_{7}
$$

Hence there exists a uniform constant $C_{8}>0$ such that

$$
H_{j} \leq H_{j}\left(z_{0}\right)=\left.\log \left(\left|S_{E}\right|^{2 \alpha} \operatorname{tr}_{\omega_{0}}\left(\omega_{j}\right)\right)\right|_{z=z_{0}}-\varphi_{j}\left(z_{0}\right)+\epsilon_{0} \log \left|S_{D}\right|_{h_{D}}^{2}\left(z=z_{0}\right) \leq C_{8} .
$$

The theorem is proved since

$$
\operatorname{tr}_{\omega_{0}}\left(\omega_{j}\right) \leq\left|S_{E}\right|_{h_{E}}^{-2 \alpha} \exp \left(H_{j}+A \Phi_{j}\right) \leq \frac{C_{9}}{\left|S_{E}\right|_{h_{E}}^{2 \alpha}\left|S_{D}\right|_{h_{D}}^{2 \beta}} .
$$

\section{Step 4. $C^{k}$ estimates.}

From the second-order estimates, the Monge-Ampère equation (3.25) is uniformly elliptic on any compact set of $Y \backslash(E \cup D)$. The $C^{k}$-estimates are local estimates and can be derived by standard Schauder estimates and elliptic estimates. Therefore for any compact subset $K$ of $Y \backslash(E \cup D)$, there exist constants $C_{K, R}$ such that

$$
\left\|\varphi_{j}\right\|_{C^{R}(K)} \leq C_{R, K}
$$

\section{Step 5.}

Proof of Theorem 3.2. By taking a sequence, we can assume $\varphi_{j} \rightarrow \varphi_{\infty} \in L^{1}\left(Y, \omega_{0}^{\kappa}\right)$. Therefore

$$
\varphi_{\infty} \in C^{0}(Y) \cap C^{\infty}(Y \backslash(E \cup D)) .
$$

On the other hand, one can choose different divisors $D$ such that $[\hat{\chi}]-\epsilon[D]>0$ for all sufficiently small $\epsilon>0$ and the intersection of such divisors is contained in $E$. Therefore $\varphi_{\infty} \in C^{\infty}(Y \backslash E)$. Each fibre of the resolution $\pi$ is connected and so $\varphi_{\infty}$ is constant along the fibre since $\varphi_{\infty} \in P S H(Y, \hat{\chi}) \cap C^{0}(Y)$ and $\hat{\chi} \geq 0$. Therefore $\varphi_{\infty}$ descends to a solution $\varphi \in P S H\left(X_{\text {can }}, \chi\right) \cap C^{0}\left(X_{c a n}\right)$ solving equation (3.22) as in Theorem 3.2, Furthermore, $\varphi$ is smooth on $X_{c a n}^{\circ}$. This completes the proof of Theorem 3.2

\section{Canonical measures on projective manifolds of nonnegative KODAIRA DIMENSION}

\subsection{Canonical measures on surfaces of nonnegative Kodaira dimension.}

Let $X$ be a Kähler surface of positive Kodaira dimension and $X_{\min }$ be its minimal model derived by $\pi: X \rightarrow X_{\min }$ contracting all the (-1)-curves $E=\bigcup_{i} E_{i}$.

If $\operatorname{kod}(X)=2, X$ is a surface of general type. Let $\Phi: X_{\min } \rightarrow X_{\text {can }}$ be a holomorphic canonical map from $X_{\min }$ to its canonical model $X_{c a n}$ with possible orbifold singularities. There exists a unique smooth orbifold Kähler-Einstein metric $\omega^{\dagger}$ on $X_{\text {can }}$. We define

$$
\omega_{K E}=(\pi \circ \Phi)^{*} \omega^{\dagger}+\sqrt{-1} \partial \bar{\partial} \log |E|^{2} .
$$

Choose a smooth positive $(1,1)$-form $\omega \in-c_{1}\left(X_{\min }\right)$ and a smooth volume form $\Omega$ with $\sqrt{-1} \partial \bar{\partial} \log \Omega=\omega$. Then $\Phi^{*} \omega^{\dagger}=\omega+\sqrt{-1} \partial \bar{\partial} \varphi$ for some $\varphi \in C^{0}\left(X_{\text {min }}\right)$ satisfying the following Monge-Ampère equation:

$$
\frac{(\omega+\sqrt{-1} \partial \bar{\partial} \varphi)^{2}}{\Omega}=e^{\varphi} .
$$


Furthermore, $\varphi$ is smooth outside the exceptional locus of the pluricanonical system. Let $\Omega_{K E}=\pi^{*}\left(e^{\varphi} \Omega\right)$ be the pullback of the unique holomorphic Kähler-Einstein volume from its canonical model. It is a continuous measure on $X$ vanishing exactly on $E$ of order one. Then $\omega_{K E}=-\operatorname{Ric}\left(\Omega_{K E}\right)$ on $X$ and $\omega_{K E}^{2}=\Omega_{K E}$ on $X \backslash E$.

Proposition 4.1. Let $X$ be a Kähler surface of general type. Then

(1) $\omega_{K E} \in-2 \pi c_{1}(X)$,

(2) $\operatorname{Ric}\left(\omega_{K E}\right)=-\omega_{K E}$ on $X \backslash E$,

(3) $h_{K E}=\Omega_{K E}^{-1}$ is an analytic Zariski decomposition for $K_{X}$.

Let $\pi: X \rightarrow X_{c a n}$ be the morphism from the general type surface $X$ to its canonical model. $\Omega_{K E}$ in the above proposition can be derived by pulling back the Kähler-Einstein volume form on $X_{c a n}$ and $\omega_{K E}=\sqrt{-1} \partial \bar{\partial} \log \Omega_{K E}$. It is easy to check that $\Omega_{K E}$ and $\omega_{K E}$ satisfy the statements in Proposition 4.1 .

If $\operatorname{kod}(X)=1, X$ is an elliptic surface. Let $\Phi: X_{\text {min }} \rightarrow X_{\text {can }}$ be the holomorphic pluricanonical fibration from $X_{m i n}$ to its canonical model $X_{c a n}$. By Theorem 3.1 in Section 3.2, there exists a canonical metric $\omega^{\dagger}$ on $X_{\text {can }}$ and a canonical measure $\Omega^{\dagger}$ on $X_{\text {min }}$ such that $\Phi^{*}\left(\omega^{\dagger}\right) \in-c_{1}\left(X_{\text {min }}\right)$ and $\operatorname{Ric}\left(\Omega^{\dagger}\right)=-\Phi^{*}\left(\omega^{\dagger}\right)$. Let $\Omega_{c a n}=$ $\pi^{*}\left(\Omega^{\dagger}\right)$ and $\omega_{\text {can }}=-\operatorname{Ric}\left(\Omega_{c a n}\right)$. It is straightforward to check that $\Omega_{c a n}$ and $\omega_{c a n}$ satisfy the following proposition.

Proposition 4.2. Let $X$ be a Kähler surface of Kodaira dimension 1. Then:

(1) $\omega_{\text {can }} \in-2 \pi c_{1}(X)$.

(2) $\omega_{\text {can }}=(\Phi \circ \pi)^{*}\left(\omega^{\dagger}\right)$ on $X \backslash E$.

(3) $h_{\text {can }}=\Omega_{\text {can }}^{-1}$ is an analytic Zariski decomposition for $K_{X}$.

4.2. Ricci-flat metrics on Kähler manifolds of zero Kodaira dimension. There have been many interesting results on singular Ricci-flat metrics. In the article EyGuZe1, singular Ricci-flat metrics are studied on normal Kähler spaces. In [To, singular Ricci-flat metrics are derived as the limit of smooth Ricci-flat Kähler metrics along certain degenerations of Kähler classes. In this section, we construct singular Ricci-flat metrics on projective manifolds of Kodaira dimension 0 as an immediate application of Theorem 2.4.

Let $X$ be an $n$-dimensional projective manifold of Kodaira dimension 0. Suppose $L \rightarrow X$ is a holomorphic line bundle such that $L$ is big and semi-ample. There exists a big smooth semi-positive $(1,1)$-form $\omega \in c_{1}(L)$. Let $\eta \in H^{0}\left(X, K_{X}^{m}\right)$ be the holomorphic $m$-tuple $n$-form for some $m \in N\left(K_{X}\right)$. Let

$$
\Omega=\frac{[\omega]^{n}}{\int_{X}(\eta \otimes \bar{\eta})^{\frac{1}{m}}}(\eta \otimes \bar{\eta})^{\frac{1}{m}}
$$

be a smooth $(n, n)$-form on $X$ and so $\int_{X} \Omega=[\omega]^{n} . \Omega$ is independent of the choice of $m \in N\left(K_{X}\right)$ and $\eta$ because the Kodaira dimension of $X$ is $0 . \Omega$ is unique up to a scalar multiplication and it can be degenerate because $K_{X}$ is not necessarily nef.

Consider the following degenerate Monge-Ampère equation:

$$
(\omega+\sqrt{-1} \partial \bar{\partial} \varphi)^{n}=\Omega .
$$

By Theorem 2.4, there exists a continuous solution $\varphi$ to equation (4.29) unique up to a constant. Let $\omega_{C Y}=\omega+\sqrt{-1} \partial \bar{\partial} \varphi$.

Proposition 4.3. $\omega_{C Y}$ is the unique closed semi-positive $(1,1)$-current in $c_{1}(L)$ with continuous local potential such that $\omega_{C Y}^{n}=\Omega$ and therefore, outside the base 
locus of the pluricanonical system,

$$
\operatorname{Ric}\left(\omega_{C Y}\right)=0 \text {. }
$$

Furthermore, $\omega_{C Y}$ is smooth on a Zariski open set of $X$.

4.3. Kähler-Einstein metrics on projective manifolds of general type. In this section, we will prove Theorem B.1. Let $X$ be an $n$-dimensional nonsingular projective variety of general type.

We choose a sequence of resolution of indeterminacies of the pluricanonical systems $\Phi_{m !}$,

$$
X \stackrel{\pi_{m_{0}}}{\longleftarrow} X_{m_{0}} \stackrel{\pi_{m_{0}+1}}{\longleftarrow} X_{m_{0}+1} \stackrel{\pi_{m_{0}+2}}{\longleftarrow} \cdots \stackrel{\pi_{m}}{\longleftarrow} X_{m} \stackrel{\pi_{m+1}}{\longleftarrow} X_{m+1} \stackrel{\pi_{m+2}}{\longleftarrow} \cdots
$$

for $m_{0}$ sufficiently large, such that:

(1)

$$
\left(\bar{\pi}_{m}\right)^{*}\left(m ! K_{X}\right)=L_{m}+E_{m}
$$

where $\bar{\pi}_{m}=\pi_{m_{0}} \circ \pi_{m_{0}+1} \circ \ldots \circ \pi_{m-1} \circ \pi_{m}$.

$$
E_{m}=\sum_{j} c_{m, j} E_{m, j}
$$

is the fixed part of $\left|\bar{\pi}_{m}^{*}\left(m ! K_{X}\right)\right|$ with each $E_{m, j}$ being a divisor with simple normal crossings.

(3) $L_{m}$ is a globally generated line bundle on $X_{m}$.

Let $\left\{\sigma_{m, j}\right\}_{j=0}^{d_{m}}$ be a basis of $H^{0}\left(X, m ! K_{X}\right)$ and $\left\{\zeta_{m, j}\right\}_{j=0}^{d_{m}}$ be a basis of $H^{0}\left(X_{m}\right.$, $L_{m}$ ) such that

$$
\pi_{m}^{*} \sigma_{m, j}=\zeta_{m, j} E_{m}
$$

We can consider $\left|\sigma_{m, j}\right| \frac{2}{m !}$ as a smooth $(n, n)$-form on $X$ as $\sigma_{m, j} \in m ! K_{X}$. Let $\Omega_{m}=\left(\sum_{j=0}^{d_{m}}\left|\sigma_{m, j}\right|^{2}\right)^{\frac{1}{m !}}$ and then $\pi_{k}^{*} \Omega_{m}$ is a smooth and possibly degenerate volume form on $X_{k}$. For simplicity we also use $\Omega_{m}$ for $\left(\pi_{k}\right)^{*}\left(\Omega_{m}\right)$ for all $k \geq m_{0}$.

The following lemma is obvious by the construction of $\Omega_{m}$.

Lemma 4.1. On $X$ and so on $X_{k}$ for $k \geq m_{0}$,

$$
\frac{\Omega_{k}}{\Omega_{k+1}}<\infty
$$

Define a smooth closed semi-positive $(1,1)$-form $\omega_{m}$ on $X_{m}$ by

$$
\omega_{m}=\frac{1}{m !} \sqrt{-1} \partial \bar{\partial} \log \left(\sum_{j=0}^{d_{m}}\left|\zeta_{m, j}\right|^{2}\right) .
$$

Obviously $\sqrt{-1} \partial \bar{\partial} \log \left(\sum_{j=0}^{d_{m}}\left|\zeta_{m, j}\right|^{2}\right)$ is the pullback of the Fubini-Study metric on $\mathbf{C P}^{d_{m}}$ from the linear system $\left|L_{m}\right|$.

Theorem 4.1. Let $R_{m}$ be the exceptional locus of the linear systems associated to $L_{m}$. There exists a unique solution $\varphi_{m} \in C^{0}\left(X_{m}\right) \cap C^{\infty}\left(X_{m} \backslash\left(\bigcup_{j} E_{m, j} \cup R_{m}\right)\right)$ to the following Monge-Ampère equation:

$$
\left(\omega_{m}+\sqrt{-1} \partial \bar{\partial} \varphi_{m}\right)^{n}=e^{\varphi} \Omega_{m} .
$$


Therefore $\omega_{K E, m}=\omega_{m}+\sqrt{-1} \partial \bar{\partial} \varphi_{m}$ is a Kähler-Einstein current on $X_{m}$ satisfying

(1) $\omega_{K E, m}$ is a positive current on $X_{m}$ and strictly positive on $X_{m} \backslash\left(\bigcup_{j} E_{m, j}\right.$ $\left.\cup R_{m}\right)$,

(2) $\operatorname{Ric}\left(\omega_{K E, m}\right)=-\omega_{K E, m}$ on $X_{m} \backslash\left(\bigcup_{j} E_{m, j}\right)$.

Proof. Let $F_{m}=\frac{\Omega_{m}}{\left(\omega_{m}\right)^{n}} . F_{m}$ has at worst pole singularities on $X_{m}$ and

$$
\int_{X_{m}}\left(F_{m}\right)^{1+\epsilon}\left(\omega_{m}\right)^{n}=\int_{X_{m}}\left(F_{m}\right)^{\epsilon} \Omega_{m}<\infty .
$$

By Theorem 2.4, there exists a unique $\varphi_{m} \in P S H\left(X_{m}, \omega_{m}\right) \cap C^{0}\left(X_{m}\right)$ solving the equation (4.32).

Also $L_{m}$ is a semi-ample line bundle and furthermore it is big. Then by Kodaira's Lemma, there exists a divisor $\left[F_{m}\right]$ such that

$$
\left[L_{m}\right]-\epsilon\left[F_{m}\right]=\bar{\pi}_{m}^{*}\left[m ! K_{X}\right]-\sum_{j} c_{m, j}\left[E_{m, j}\right]-\epsilon\left[F_{m}\right]
$$

is ample for sufficiently small $\epsilon>0$. By a similar argument in the proof of Theorem 3.2, we can show that $\varphi_{m} \in C^{\infty}\left(X_{m} \backslash\left(\bigcup_{j} E_{m, j} \cup R_{m}\right)\right)$.

Corollary 4.1. $e^{\varphi_{m}} \Omega_{m}$ descends from $X_{m}$ to a continuous measure on $X$ and $h_{m}=e^{-\varphi_{m}} \Omega_{m}^{-1}$ is a singular Hermitian metric on $X$ with its curvature $\Theta_{h_{m}} \geq 0$. Furthermore, on $X \backslash B s\left(\left|m_{0} ! K_{X}\right|\right)$,

$$
\left(\sqrt{-1} \partial \bar{\partial} \log \Omega_{m}+\sqrt{-1} \partial \bar{\partial} \varphi_{m}\right)^{n}=e^{\varphi_{m}} \Omega_{m} .
$$

Proof. $e^{\varphi_{m}} \Omega_{m}$ can be pulled back as a continuous volume form on $X \backslash B s\left(m ! K_{X}\right)$. On the other hand, $\varphi_{m}$ is uniformly bounded in $L^{\infty}(X)$, also $\Omega_{m}$ is smooth on $X$ and vanishes exactly on $B s\left(\left|m ! K_{X}\right|\right)$. Therefore $e^{\varphi_{m}} \Omega_{m}$ is continuous on $X$.

$B s\left(\left|m ! K_{X}\right|\right)$ is a complete closed pluripolar set on $X$ using $H^{0}\left(X, m ! K_{X}\right)$. Since $\log \Omega_{m}+\varphi_{m}$ is uniformly bounded above and $\sqrt{-1} \partial \bar{\partial} \log \Omega_{m}+\sqrt{-1} \partial \bar{\partial} \varphi_{m}$ is a positive closed $(1,1)$ current on $X \backslash B s\left(\left|m_{0} K_{X}\right|\right), \sqrt{-1} \partial \bar{\partial} \log \Omega_{m}+\sqrt{-1} \partial \bar{\partial} \varphi_{m}$ extends to a positive closed current on $X$ using a local argument.

Equation (4.33) is then derived directly from Equation (4.32).

Although $\Theta_{h_{m}}$ is a singular Kähler-Einstein metric on $X$, but without assuming a finite generation of canonical rings, $\left(K_{X}, h_{m}\right)$ is not necessarily an analytic Zariski decomposition. One has to let $m$ tend to infinity in order for $e^{\varphi_{m}} \Omega_{m}$ to have the least vanishing order.

Let $D$ be an ample divisor on $X$ such that $K_{X}+D$ is ample. Then there exists a Hermitian metric $h_{D}$ on the line bundle induced by $[D]$ such that $\omega_{0}-$ $\sqrt{-1} \partial \bar{\partial} \log h_{D}>0$. We can also assume that $D$ contains the base locus of all $\left|m ! K_{X}\right|$ for $m \geq m_{0}$.

Lemma 4.2. Let $\Omega_{0}$ be a smooth and nowhere-vanishing volume form on $X$. Then there exists a constant $C>0$ such that for each $m \geq m_{0}$,

$$
e^{\varphi_{m}} \Omega_{m} \leq C \Omega_{0} \text {. }
$$

Proof. Let $\chi_{0}=\sqrt{-1} \partial \bar{\partial} \log \Omega_{0}$. Let $D_{m}$ be a divisor on $X$ such that on $X \backslash D_{m}, \varphi_{m}$ is smooth and $\Omega_{m}$ is strictly positive. Let $D$ be an ample divisor on $X$. Let $S_{D_{m}}$ be a defining function and $h_{D_{m}}$ be a smooth Hermitian metric on the line bundle 
associated to $\left[D_{m}\right]$. Let $S_{D}$ be a defining function and $h_{D}$ be a smooth positively curved Hermitian metric on the line bundle associated to $[D]$.

Let $\theta_{D_{m}}=-\sqrt{-1} \partial \bar{\partial} \log h_{D_{m}}$ and $\theta_{D}=-\sqrt{-1} \partial \bar{\partial} \log h_{D}>0$. We also define

$$
\psi_{m, \epsilon}=\varphi_{m}+\log \frac{\Omega_{m}}{\Omega_{0}}+\epsilon^{2} \log \left|S_{D_{m}}\right|_{h_{m}}^{2}+\epsilon \log \left|S_{D}\right|_{h_{D}}^{2} .
$$

For simplicity, we use $\chi_{0}$ and $\Omega_{0}$ for $\left(\bar{\pi}_{m}\right)^{*} \chi_{0}$ and $\left(\bar{\pi}_{m}\right)^{*} \Omega_{0}$. Notice that $\chi_{0}$ is not necessarily positive and $\Omega_{0}$ might vanish somewhere on $X_{m}$. Then outside $D_{m}$ and $D, \psi_{m, \epsilon}$ satisfies the following equation:

$$
\frac{\left(\chi_{0}+\epsilon^{2} \theta_{D_{m}}+\epsilon \theta_{D}+\sqrt{-1} \partial \bar{\partial} \log \psi_{m, \epsilon}\right)^{n}}{\Omega_{0}}=\left|S_{D_{m}}\right|_{h_{D_{m}}}^{-2 \epsilon^{2}}\left|S_{D}\right|_{h_{D}}^{-2 \epsilon} e^{\psi_{m, \epsilon}} .
$$

It is easy to see that $\psi_{m, \epsilon}$ tends to $-\infty$ near $D_{m}$ and $D$, and for $\epsilon>0$ sufficiently small, $\theta_{D}+\epsilon \theta_{D_{m}}>0$. By the maximum principle,

$$
e^{\psi_{m, \epsilon}} \leq \max _{X}\left(\left|S_{D_{m}}\right|_{h_{D_{m}}}^{2 \epsilon^{2}}\left|S_{D}\right|_{h_{D}}^{2 \epsilon} \frac{\left(\chi_{0}+\epsilon^{2} \theta_{D_{m}}+\epsilon \theta_{D}\right)^{n}}{\Omega_{0}}\right)
$$

and

$$
e^{\varphi_{m}} \frac{\Omega_{m}}{\Omega_{0}} \leq\left|S_{D_{m}}\right|_{h_{D_{m}}}^{-2 \epsilon^{2}}\left|S_{D}\right|_{h_{D}}^{-2 \epsilon} \max _{X}\left(\left|S_{D_{m}}\right|_{h_{D_{m}}}^{2 \epsilon^{2}}\left|S_{D}\right|_{h_{D}}^{2 \epsilon} \frac{\left(\chi_{0}+\epsilon^{2} \theta_{D_{m}}+\epsilon \theta_{D}\right)^{n}}{\Omega_{0}}\right) .
$$

Now we let $\epsilon$ tend to 0 , and

$$
e^{\varphi_{m}} \frac{\Omega_{m}}{\Omega_{0}} \leq \max _{X}\left(\frac{\left(\chi_{0}\right)^{n}}{\Omega_{0}}\right) .
$$

Proposition 4.4. $e^{\varphi_{m}} \Omega_{m}$ is increasing, that is, on $X$,

$$
e^{\varphi_{m}} \Omega_{m} \leq e^{\varphi_{m+1}} \Omega_{m+1} .
$$

Proof. We shall compare $e^{\varphi_{m}} \Omega_{m}$ and $e^{\varphi_{m+1}} \Omega_{m+1}$ on $X_{m+1}$. Let

$$
U_{(m+1)}=\left\{\begin{array}{l|l}
s \in X_{m+1} & \frac{\Omega_{m+1}}{\Omega_{m}}<\infty
\end{array}\right\} .
$$

$\varphi_{m}$ and $\varphi_{m+1}$ are the solutions of

$$
\left(\omega_{m}+\sqrt{-1} \partial \bar{\partial} \varphi_{m}\right)^{n}=e^{\varphi_{m}} \Omega_{m}
$$

and

$$
\left(\omega_{m+1}+\sqrt{-1} \partial \bar{\partial} \varphi_{m+1}\right)^{n}=e^{\varphi_{m+1}} \Omega_{m+1} .
$$

Define

and

$$
\psi=\varphi_{m+1}-\varphi_{m}+\log \frac{\Omega_{m+1}}{\Omega_{m}}
$$

$$
V=\left\{z \in X_{m+1} \mid \psi<0\right\}
$$

It is easy to see that

$$
V \subset U_{m+1}
$$

since both $\varphi_{m+1}$ and $\varphi_{m}$ are in $L^{\infty}\left(X_{m+1}\right)$. 
On $U_{m+1}, \log \left(\frac{\left(E_{m+1}\right)^{m !}}{\left(\left(\pi_{m+1}\right)^{*} E_{m}\right)^{(m+1) !}}\right)$ is holomorphic and so

$$
\begin{aligned}
\omega_{m+1}+ & \sqrt{-1} \partial \bar{\partial} \varphi_{m+1} \\
= & \omega_{m}+\sqrt{-1} \partial \bar{\partial} \varphi_{m}+\sqrt{-1} \partial \bar{\partial} \log \left(\varphi_{m+1}-\varphi_{m}\right) \\
& +\sqrt{-1} \partial \bar{\partial} \log \left(\frac{\left(\sum_{j=0}^{d_{m+1}}\left|\zeta_{m+1, j}\right|^{2}\right)^{\frac{1}{(m+1) !}}}{\left(\sum_{j=0}^{d_{m}}\left|\left(\pi_{m+1}\right)^{*} \zeta_{m, j}\right|^{2}\right)^{\frac{1}{m !}}}\right) \\
= & \omega_{m}+\sqrt{-1} \partial \bar{\partial} \varphi_{m}+\sqrt{-1} \partial \bar{\partial} \psi+\sqrt{-1} \partial \bar{\partial} \log \left(\frac{\left|E_{m+1}\right|^{\frac{2}{(m+1) !}}}{\left|\left(\pi_{m+1}\right)^{*} E_{m}\right|^{\frac{2}{m !}}}\right) \\
= & \omega_{m}+\sqrt{-1} \partial \bar{\partial} \varphi_{m}+\sqrt{-1} \partial \bar{\partial} \psi .
\end{aligned}
$$

Hence $\psi$ satisfies the following equation on $U_{m+1}$ :

$$
\frac{\left(\omega_{m}+\sqrt{-1} \partial \bar{\partial} \varphi_{m}+\sqrt{-1} \partial \bar{\partial} \psi\right)^{n}}{\left(\omega_{m}+\sqrt{-1} \partial \bar{\partial} \varphi_{m}\right)^{n}}=e^{\psi} .
$$

By the comparison principle,

$$
\begin{aligned}
\int_{V}\left(\omega_{m}+\sqrt{-1} \partial \bar{\partial} \varphi_{m}\right)^{n} \\
\quad \leq \int_{V}\left(\omega_{m}+\sqrt{-1} \partial \bar{\partial} \varphi_{m}+\sqrt{-1} \partial \bar{\partial} \psi\right)^{n} \\
\quad=\int_{V} e^{\psi}\left(\omega_{m}+\sqrt{-1} \partial \bar{\partial} \varphi_{m}\right)^{n} .
\end{aligned}
$$

Therefore $\Psi=0$ on $V$ due to the fact that it is continuous on $V$, and so on $X_{m+1}$,

$$
\psi=0 \text {. }
$$

This completes the proof.

Proposition 4.5. There exists a measure $\Omega_{K E}$ on $X$ such that

(1) $\Omega_{K E}=\lim _{m \rightarrow \infty} e^{\varphi_{m}} \Omega_{m}$.

(2) $\left(K_{X}, \Omega_{K E}^{-1}\right)$ is an analytic Zariski decomposition. Furthermore, on $X$,

$$
\frac{\Omega_{K E}}{\Omega_{0}}<\infty \text { and } \frac{\Psi_{X, \epsilon}}{\Omega_{K E}}<\infty,
$$

where $\Psi_{X, \epsilon}$ is defined as (1.7).

(3) $\omega_{K E}=\sqrt{-1} \partial \bar{\partial} \log \Omega_{K E} \in-2 \pi c_{1}(X)$ is a closed positive Kähler-Einstein current and on $X \backslash B s\left(X, K_{X}\right)$,

$$
\left(\omega_{K E}\right)^{n}=\Omega_{K E} \text { and so } \operatorname{Ric}\left(\omega_{K E}\right)=-\omega_{K E} .
$$

Proof. By Lemma 4.2 and Proposition 4.4, we can define $\Omega_{K E}$ as

$$
\Omega_{K E}=\lim _{m \rightarrow \infty} e^{\varphi_{m}} \Omega_{m}
$$

and $\frac{\Omega_{K E}}{\Omega_{0}}$ is uniformly bounded from above. Since $\varphi_{m}+\log \frac{\Omega_{m}}{\Omega_{0}} \in \operatorname{PSH}\left(X, \chi_{0}\right)$, $\left\{\varphi_{m}+\log \frac{\Omega_{m}}{\Omega_{0}}\right\}_{m=m_{0}}^{\infty}$ is convergent in $L^{1}(X)$. Also $P S H\left(X, \chi_{0}\right) \cap L^{1}(X)$ is closed 
in $L^{1}(X)$. Therefore

$$
\lim _{m \rightarrow \infty}\left(\varphi_{m}+\log \frac{\Omega_{m}}{\Omega_{0}}\right)=\log \frac{\Omega_{K E}}{\Omega_{0}}
$$

in $P S H\left(X, \chi_{0}\right) \cap L^{1}(X)$ and $\log \frac{\Omega_{K E}}{\Omega_{0}} \in P S H\left(X, \chi_{0}\right) \cap L^{1}(X)$.

Let $h_{K E}=\Omega_{K E}^{-1}$ be the Hermitian metric on $K_{X}$. By the construction of $\Omega_{m}$,

$$
|\sigma|_{h_{K E}^{m}}^{2}<\infty
$$

for any section $\sigma \in H^{0}\left(X, m K_{X}\right)$. Therefore $\frac{\Psi_{X, \epsilon}}{\Omega_{K E}}<\infty$ and so

$$
H^{0}\left(X, \mathcal{O}_{X}\left(m K_{X}\right) \otimes \mathcal{I}\left(h_{K E}^{m}\right)\right) \rightarrow H^{0}\left(X, \mathcal{O}_{X}\left(m K_{X}\right)\right)
$$

is an isomorphism and $\left(K_{X}, h_{K E}\right)$ is an analytic Zariski decomposition.

Since $\varphi_{m}+\log \frac{\Omega_{m}}{\Omega_{0}}$ converges uniformly on any compact set of $X \backslash B s\left(X, K_{X}\right)$ to $\log \frac{\Omega_{K E}}{\Omega_{0}}$, we have on $X \backslash B s\left(X, K_{X}\right)$,

$\left(\sqrt{-1} \partial \bar{\partial} \log \Omega_{K E}\right)^{n}=\lim _{m \rightarrow \infty}\left(\sqrt{-1} \partial \bar{\partial} \log \Omega_{m}+\sqrt{-1} \partial \bar{\partial} \varphi_{m}\right)^{n}=\lim _{m \rightarrow \infty} e^{\varphi_{m}} \Omega_{m}=\Omega_{K E}$.

This concludes the proof of the proposition as well as Theorem B.1.

Remark 4.1. The existence of such a canonical metric does not depend on the finite generation of the canonical ring of $X$. The regularity and uniqueness of such Kähler-Einstein metrics will be investigated in our future study.

4.4. Projective manifolds of positive Kodaira dimension. Let $X$ be an $n$ dimensional nonsingular projective variety of Kodaira dimension $\kappa$, where $0<$ $\kappa<n$. Let $\Phi^{\dagger}: X^{\dagger} \rightarrow Y^{\dagger}$ be the Iitaka fibration of $X$ unique up to birational equivalence. Let $\Phi_{m}$ be the pluricanonical map associated to the linear system $\left|m K_{X}\right|$. Then for $m$ sufficiently large there exists a commutative diagram

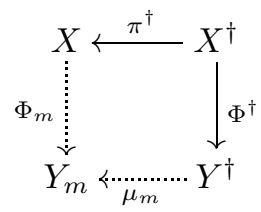

as in Section 2.2. A very general fibre of $\Phi^{\dagger}$ has Kodaira dimension zero.

We will generalize the notion of the Weil-Petersson metric on a special local deformation space of Kähler manifolds of zero Kodaira dimension.

Definition 4.1. Let $f: \mathcal{X} \rightarrow B$ be a holomorphic nonsingular fibration over a ball $B \in \mathbf{C}^{\kappa}$ such that for any $t \in B, \mathcal{X}_{t}=f^{-1}(t)$ is a nonsingular fibre of dimension $n-\kappa$. Let $t=\left(t_{1}, \ldots, t_{\kappa}\right)$ be the holomorphic coordinates of $B$, where $\kappa=\operatorname{dim} \mathcal{M}$. Then each $\frac{\partial}{\partial t_{i}}$ corresponds to an element $\iota\left(\frac{\partial}{\partial t_{i}}\right) \in H^{1}\left(\mathcal{X}_{t}, T_{\mathcal{X}_{t}}\right)$ through the KodairaSpencer map $\iota$. We assume that there exists a nontrivial holomorphic $(n-\kappa, 0)$-form $\Psi$ on $\mathcal{X}$ such that its restriction on each fibre $\mathcal{X}_{t}$ is also a nontrivial holomorphic $(n-\kappa, 0)$-form on $\mathcal{X}$. Then the Weil-Petersson metric is defined by the $L^{2}$-inner product

$$
\left(\frac{\partial}{\partial t_{i}}, \frac{\partial}{\partial \bar{t}_{j}}\right)_{\omega_{W P}}=\frac{\left.\int_{\mathcal{X}_{t}} \Psi\right\lrcorner \iota\left(\frac{\partial}{\partial t_{i}}\right) \wedge \overline{\Psi\lrcorner \iota\left(\frac{\partial}{\partial t_{i}}\right)}}{\int_{\mathcal{X}_{t}} \Psi \wedge \bar{\Psi}},
$$

where $\Psi\lrcorner \iota\left(\frac{\partial}{\partial t_{i}}\right)$ is the contraction of $\Psi$ and $\iota\left(\frac{\partial}{\partial t_{i}}\right)$. 
The metric defined above is a pseudometric and the associated closed $(1,1)$-form $\omega_{W P}$ is only semi-positive in general. In Definition 4.1, $\omega_{W P}$ depends on the choice of the holomorphic $(n-\kappa, 0)$-form $\Psi$, however, it is uniquely determined for the Iitaka fibration $\Phi^{\dagger}: X^{\dagger} \rightarrow Y^{\dagger}$ by the following lemma.

Lemma 4.3. The Weil-Petersson metric is well defined on a Zariski open set of $Y^{\dagger}$ and it is unique.

Proof. Let $B \subset Y^{\dagger}$ be a nonsingular open neighborhood such that each fibre over $B$ is nonsingular. Let $\mathcal{X}=\left(\Phi^{\dagger}\right)^{-1}(B) \subset X^{\dagger}$. Without loss of generality, we assume $H^{0}\left(X^{\dagger}, K_{X^{\dagger}}\right) \neq \phi$; otherwise we can replace $K_{X}$ by a sufficiently large power of $K_{X}$, and so $H^{0}\left(B,\left(\Phi^{\dagger}\right)_{*} \Omega_{\mathcal{X} / B}^{n-\kappa}\right) \neq \phi$. Then the assumption in Definition 4.1 can be satisfied. Let $\Psi_{1}$ and $\Psi_{2}$ be two holomorphic $(n-\kappa)$-forms over $B$ in Definition 4.1 . Since a very general fibre has Kodaira dimension zero, for a general point $t \in B$,

$$
\left.\frac{\Psi_{1}}{\Psi_{2}}\right|_{\mathcal{X}_{t}}=\text { constant }
$$

and so $\left.\frac{\Psi_{1}}{\Psi_{2}}\right|_{\mathcal{X}_{t}}$ is constant on each point $t \in B$ since $\left.\frac{\Psi_{1}}{\Psi_{2}}\right|_{\mathcal{X}_{t}}$ is smooth on $\mathcal{X}$.

One can also represent $\omega_{W P}$ as the curvature form of the first Hodge bundle $f_{*} \Omega_{\mathcal{X} / B}^{n-\kappa}$ with the same assumption as in Definition 4.1, Letting $\Psi$ be a nonzero local holomorphic section of $f_{*} \Omega_{\mathcal{X} / B}^{n-\kappa}$, one can define the Hermitian metric $h_{W P}$ on $f_{*} \Omega_{\mathcal{X} / B}^{n-\kappa}$ by

$$
\left|\Psi_{t}\right|_{h_{W P}}^{2}=\int_{\mathcal{X}_{t}} \Psi_{t} \wedge \overline{\Psi_{t}}
$$

Then the Weil-Petersson metric is given by

$$
\omega_{W P}=\operatorname{Ric}\left(h_{W P}\right) .
$$

Therefore the Weil-Petersson metric is unique on $B$.

We choose a sequence of resolution of indeterminacies of the pluricanonical systems $\Phi_{m !}=\Phi_{\left|m ! K_{X}\right|}$,

$$
X \stackrel{\pi_{m_{0}}}{\longleftarrow} X_{m_{0}} \stackrel{\pi_{m_{0}+1}^{\longleftarrow}}{\longleftarrow} X_{m_{0}+1} \stackrel{\pi_{m_{0}+2}}{\longleftarrow} \cdots \stackrel{\pi_{m}}{\longleftarrow} X_{m} \stackrel{\pi_{m+1}}{\longleftarrow} X_{m+1} \stackrel{\pi_{m+2}}{\longleftarrow} \cdots
$$

for $m_{0}$ sufficiently large, such that

(1)

$$
\left(\bar{\pi}_{m}\right)^{*}\left(m ! K_{X}\right)=L_{m}+E_{m},
$$

(2)

where $\bar{\pi}_{m}=\pi_{m} \circ \pi_{m-1} \circ \ldots \circ \pi_{m_{0}}$.

$$
E_{m}=\sum_{j} c_{m, j} E_{m, j}
$$

is the fixed part of $\left|\left(\bar{\pi}_{m}\right)^{*}\left(m ! K_{X}\right)\right|$ with each $E_{m, j}$ being a divisor with simple normal crossings.

(3) $L_{m}$ is a globally generated line bundle on $X_{m}$. 
Let $Y_{m}$ be the variety determined by the pluricanonical system $\left|m ! K_{X}\right|$ and $\Psi_{m}=\Psi_{\left|L_{m}\right|}$ be the rational map associated to the linear system $\left|L_{m}\right|$. Then we have the following diagram:

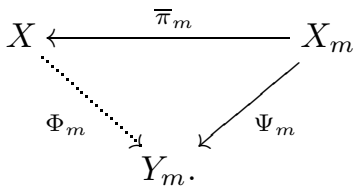

There exists a commutative diagram by choosing $m_{0}$ sufficiently large

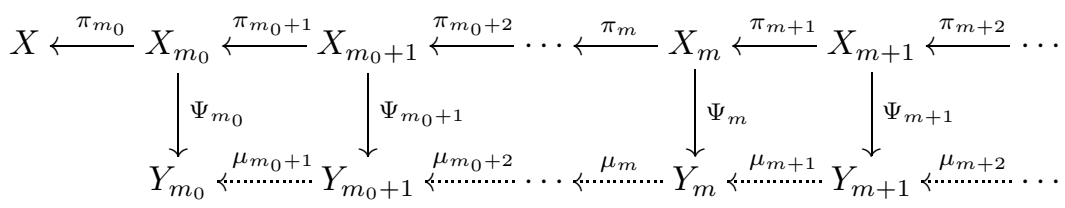

of rational maps and holomorphic maps, where the horizontal maps are birational and $\mu_{m}$ is given by the projection from $\left|m ! K_{X}\right|$ to $\left|(m-1) ! K_{X}\right|$ as a subspace of $\left|m ! K_{X}\right|$.

Let $\left\{\sigma_{m, j}\right\}_{j=0}^{d_{m}}$ be a basis of $H^{0}\left(X, m ! K_{X}\right)$ and $\left\{\zeta_{m, j}\right\}_{j=0}^{d_{m}}$ be a basis of $H^{0}\left(X, L_{m}\right)$ such that

Then

$$
\left(\bar{\pi}_{m}\right)^{*} \sigma_{m, j}=\zeta_{m, j} E_{m} \in H^{0}\left(X_{m},\left(\bar{\pi}_{m}\right)^{*}\left(m ! K_{X}\right)\right) .
$$

$$
\frac{\left(\sum_{j=0}^{d_{m}} \sigma_{m, j} \otimes \overline{\sigma_{m, j}}\right)^{\frac{1}{m !}}}{\left(\sum_{j=0}^{d_{m+1}} \sigma_{m+1, j} \otimes \overline{\sigma_{m+1, j}}\right)^{\frac{1}{(m+1) !}}}<\infty
$$

where $1+d_{m}=\operatorname{dim} H^{0}\left(X, m ! K_{X}\right)$.

Let $\Omega_{m}=\left(\sum_{j=0}^{d_{m}} \sigma_{m, j} \otimes \overline{\sigma_{m, j}}\right)^{\frac{1}{m !}}$ and $\Omega_{(m)}=\left(\Psi_{m}\right)_{*} \Omega_{m}$. For simplicity, we abuse the notation and use $\Omega_{m}$ for $\left(\bar{\pi}_{k}\right)^{*} \Omega_{m}$ for $k \geq m_{0}$ without causing confusion.

Let

$$
\omega_{m}=\frac{1}{m} \sqrt{-1} \partial \bar{\partial} \log \left(\sum_{j=0}^{d_{m}}\left|\zeta_{m, j}\right|^{2}\right)
$$

be the normalized Fubini-Study metric on $Y_{m}$. Then the same argument as in Proposition 3.2 gives the following lemma.

Lemma 4.4. There exists $p=p(m)>1$ such that

$$
F_{m}=\frac{\Omega_{(m)}}{\omega_{m}^{\kappa}} \in L^{p}\left(Y_{m}, \omega_{m}^{\kappa}\right) .
$$

The following proposition is immediate as in Section 3.2.

Proposition 4.6. There exists a unique solution $\varphi_{m} \in P S H\left(Y_{m}, \omega_{m}\right) \cap C^{0}\left(Y_{m}\right)$ to the following Monge-Ampère equation:

$$
\left(\omega_{m}+\sqrt{-1} \partial \bar{\partial} \varphi_{m}\right)^{\kappa}=e^{\varphi_{m}} \Omega_{(m)} .
$$

Furthermore, $\varphi_{m}$ is smooth on a Zariski open set of $Y_{m}$.

Lemma 4.5. There exists a Zariski open set $U$ of $Y_{m+1}$ such that $\frac{\Omega_{m}}{\Omega_{m+1}}$ is constant along each fibre of $\Psi_{m+1}$ over $U$. 
Proof. Letting $F=\frac{\Omega_{m}}{\Omega_{m+1}}$, it is easy to see that $F$ is smooth. We consider the following diagram:

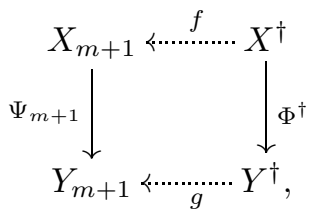

where $\Phi^{\dagger}: X^{\dagger} \rightarrow Y^{\dagger}$ is an Iitaka fibration of $X$.

A very general fiber of $\Phi^{\dagger}$ is nonsingular of Kodaira dimension 0 . Let $F_{s_{0}}=$ $\left(\Phi^{\dagger}\right)^{-1}\left(s_{0}\right)$ be a very general fibre. Consider $B=\left\{s \in Y^{\dagger}|| s-s_{0} \mid<\delta\right\}$ such that for any $s \in B, F_{s}=\left(\Phi^{\dagger}\right)^{-1}(s)$ is nonsingular. Let $\eta$ be a nowhere-vanishing holomorphic $\kappa$-form on $B$. Then

$$
\left.\frac{\left(\pi^{\dagger}\right)^{*} \sigma_{m, j}}{\eta^{m}}\right|_{s_{0}} \in H^{0}\left(F_{s_{0}}, m ! K_{F_{s_{0}}}\right)
$$

and

$$
\left.\frac{\left(\pi^{\dagger}\right)^{*} \sigma_{m+1, j}}{\eta^{m+1}}\right|_{s_{0}} \in H^{0}\left(F_{s_{0}},(m+1) ! K_{F_{s_{0}}}\right) .
$$

Since $\operatorname{dim} P H^{0}\left(F_{s_{0}}, k K_{F_{s_{0}}}\right)=0$ for any $k \geq 1$ and $\frac{\Omega_{m}}{\Omega_{m+1}}<\infty, \frac{\Omega_{m}}{\Omega_{m+1}}$ must be constant on each $F_{s_{0}}$.

Therefore $f^{*} F$ is constant on a very general fibre of $\Phi^{\dagger}$. By Hartogs' theorem, $f^{*} F$ is smooth on $X^{\dagger}$ and so $f^{*} F$ is the pullback of a function on a Zariski open set of $Y^{\dagger}$. By the commutative diagram, on a Zariski open set of $X_{m+1}, F$ is the pullback of a function on $Y_{m+1}$ and so $F$ has to be constant on a very general fibre of $\Psi_{m+1}$.

Hence $\frac{\Omega_{m}}{\Omega_{m+1}}$ descends to a function on $Y_{m+1}$. By the commutative diagram, the following lemma is immediate.

Lemma 4.6. For each $m \geq m_{0}$,

$$
\left(\Psi_{m+1}\right)_{*} \Omega_{m}=\left(\mu_{m+1}\right)^{*} \Omega_{(m)} .
$$

The following corollary is immediate by the diagram (4.42) and Lemmas 4.5 and 4.6 .

Corollary 4.2. For each $m \geq m_{0}$, on $X_{m+1}$,

$$
\frac{\Omega_{m}}{\Omega_{m+1}}=\frac{\left(\mu_{m+1}\right)^{*} \Omega_{(m)}}{\Omega_{(m+1)}} .
$$

Lemma 4.7. Let $U_{m+1}=\left\{s \in Y_{m+1} \mid \frac{\Omega_{m+1}}{\Omega_{m}}<\infty\right\}$. Then $\mu_{m+1}$ is holomorphic on $U_{m+1}$.

Proof. On $\left(\Psi_{m+1}\right)^{-1}\left(U_{m+1}\right)$,

$$
0<\frac{\left(\sum_{j}\left|\sigma_{m, j}\right|^{2}\right)^{\frac{1}{m}}}{\left(\sum_{j}\left|\sigma_{m+1, j}\right|^{2}\right)^{\frac{1}{m+1}}}<\infty .
$$


Both $L_{m+1}$ and $\left(\pi_{m}\right)^{*} L_{m}$ are globally generated. Therefore the base locus of

$$
\left\{\frac{\left(\left(\bar{\pi}_{m+1}\right)^{*} \sigma_{m, j}\right)^{m+1}}{E_{m+1}}\right\}_{j=0}^{d_{m}}
$$

is outside $U_{m+1}$ and so $\mu_{m+1}$ is well defined on $U_{m+1}$.

Proposition 4.7. For any $m \geq m_{0}$, the measure $e^{\varphi_{m}} \Omega_{(m)}$ is increasing, that is, on $Y_{m+1}$

$$
\left(\mu_{m+1}\right)^{*}\left(e^{\varphi_{m}} \Omega_{(m)}\right) \leq e^{\varphi_{m+1}} \Omega_{(m+1)} .
$$

Proof. By resolution of singularities, we can assume that $Y_{(m+1)}$ is nonsingular by replacing $Y_{m+1}$ by its nonsingular model. Let $\omega_{m}^{\prime}=\left(\mu_{m+1}\right)^{*} \omega_{m}, \varphi_{m}^{\prime}=\left(\mu_{m+1}\right)^{*} \varphi_{m}$ and $\Omega_{(m)}^{\prime}=\left(\mu_{m+1}\right)^{*} \Omega_{(m)}$. So $\varphi_{m}^{\prime}$ satisfies the following Monge-Ampère equation:

$$
\left(\omega_{m}^{\prime}+\sqrt{-1} \partial \bar{\partial} \varphi_{m}^{\prime}\right)^{\kappa}=e^{\varphi_{m}^{\prime}} \Omega_{(m)}^{\prime}
$$

on $U_{m+1}$, where the $\omega_{m}^{\prime}$ are smooth and positive, and so the Monge-Ampère mass $\left(\omega_{m}^{\prime}+\sqrt{-1} \partial \bar{\partial} \varphi_{m}^{\prime}\right)^{\kappa}$ is well defined. Also $\varphi_{m+1}$ is the solution of

$$
\left(\omega_{m+1}+\sqrt{-1} \partial \bar{\partial} \varphi_{m+1}\right)^{\kappa}=e^{\varphi_{m+1}} \Omega_{(m+1)} .
$$

Define

$$
\psi=\varphi_{m+1}-\varphi_{m}^{\prime}+\log \frac{\Omega_{(m+1)}}{\Omega_{(m)}^{\prime}}
$$

and $V=\left\{z \in Y_{m} \mid \psi \leq 0\right\}$. It is easy to see that

$$
V \subset U_{m+1}
$$

since both $\varphi_{m+1}$ and $\varphi_{m}^{\prime}$ are in $L^{\infty}\left(Y_{m+1}\right)$. In particular, $\varphi_{m}^{\prime} \in C^{0}\left(U_{m+1}\right)$.

On $U_{m+1}, \log \left(\frac{\left|E_{m+1}\right|^{2 m}}{\left|\left(\pi_{m}\right)^{*} E_{m}\right|^{2(m+1)}}\right)$ is smooth and so

$$
\begin{aligned}
\omega_{m+1}+ & \sqrt{-1} \partial \bar{\partial} \varphi_{m+1} \\
= & \omega_{m}^{\prime}+\sqrt{-1} \partial \bar{\partial} \varphi_{m}^{\prime}+\sqrt{-1} \partial \bar{\partial} \log \left(\varphi_{m+1}-\varphi_{m}^{\prime}\right) \\
& +\sqrt{-1} \partial \bar{\partial} \log \left(\frac{\left(\sum_{j}\left|\zeta_{m+1, j}\right|^{2}\right)^{\frac{1}{m+1}}}{\left(\sum_{j}\left|\left(\pi_{m}\right)^{*} \zeta_{m, j}\right|^{2}\right)^{\frac{1}{m}}}\right) \\
= & \omega_{m}^{\prime}+\sqrt{-1} \partial \bar{\partial} \varphi_{m}^{\prime}+\sqrt{-1} \partial \bar{\partial} \psi-\sqrt{-1} \partial \bar{\partial} \log \left(\frac{\left|E_{m+1}\right|^{\frac{2}{m+1}}}{\left|\left(\pi_{m}\right)^{*} E_{m}\right|^{\frac{2}{m}}}\right) \\
= & \omega_{m}^{\prime}+\sqrt{-1} \partial \bar{\partial} \varphi_{m}^{\prime}+\sqrt{-1} \partial \bar{\partial} \psi .
\end{aligned}
$$

Hence $\psi$ satisfies the following equation on $U_{m+1}$ :

$$
\frac{\left(\omega_{m}^{\prime}+\sqrt{-1} \partial \bar{\partial} \varphi_{m}^{\prime}+\sqrt{-1} \partial \bar{\partial} \psi\right)^{\kappa}}{\left(\omega_{m}^{\prime}+\sqrt{-1} \partial \bar{\partial} \varphi_{m}^{\prime}\right)^{\kappa}}=e^{\psi} .
$$


By the comparison principle, we have on $V=\left\{z \in Y_{m+1} \mid \psi(z)<0\right\} \subset U_{m+1}$,

$$
\begin{aligned}
\int_{V}\left(\omega_{m}^{\prime}+\sqrt{-1} \partial \bar{\partial} \varphi_{m}^{\prime}\right)^{\kappa} & \leq \int_{V}\left(\omega_{m}^{\prime}+\sqrt{-1} \partial \bar{\partial} \varphi_{m}^{\prime}+\sqrt{-1} \partial \bar{\partial} \psi\right)^{\kappa} \\
& =\int_{V} e^{\psi}\left(\omega_{m}^{\prime}+\sqrt{-1} \partial \bar{\partial} \varphi_{m}^{\prime}\right)^{\kappa} .
\end{aligned}
$$

Therefore $\psi=0$ on $V$ due to the fact that it is continuous on $V$, and so on $Y_{m+1}$,

$$
\psi \geq 0 \text {. }
$$

This completes the proof.

For simplicity we again use $\varphi_{m}$ for $\left(\Psi_{m}\right)^{*} \varphi_{m}$. We can consider $\varphi_{m}$ as a function on $X, X_{m}$ or $X^{\dagger}$ since they are all birationally equivalent.

Corollary 4.3. For any $m \geq m_{0}$, the measure $e^{\varphi_{m}} \Omega_{m}$ is increasing, that is,

$$
e^{\varphi_{m}} \Omega_{m} \leq e^{\varphi_{m+1}} \Omega_{m+1} .
$$

Lemma 4.8. $e^{\varphi_{m}} \Omega_{m}$ is continuous on $X$ and let $h_{m}=e^{-\varphi_{m}} \Omega_{m}^{-1}$ be the Hermitian metric on $K_{X}$. Then

$$
\Theta_{h_{m}} \geq 0
$$

Proof. Obviously, $e^{\varphi_{m}} \Omega_{m}$ is continuous on $X \backslash B s\left(\left|m ! K_{X}\right|\right)$ since the inverse of $\bar{\pi}_{m}$ is isomorphic from $X_{m} \backslash\left(\bar{\pi}_{m}\right)^{-1}\left(B s\left(\left|m ! K_{X}\right|\right)\right)$ to $X \backslash\left(B s\left(\left|m ! K_{X}\right|\right)\right.$. On the other hand, $\varphi_{m} \in L^{\infty}(X)$ and $\Omega_{m}$ vanishes exactly on $B s\left(\left|m ! K_{X}\right|\right)$. Hence $e^{\varphi_{m}} \Omega_{m}$ is continuous.

$\sqrt{-1} \partial \bar{\partial} \log \Omega_{m}+\sqrt{-1} \partial \bar{\partial} \varphi_{m} \geq 0$ on $X \backslash B s\left(\left|m ! K_{X}\right|\right)$ and $B s\left(\left|m ! K_{X}\right|\right)$ is a closed complete pluripolar set of $X$. By the fact that $e^{\varphi_{m}} \Omega_{m}$ is bounded above on $X$,

$$
\Theta_{h_{m}}=\sqrt{-1} \partial \bar{\partial} \log \Omega_{m}+\sqrt{-1} \partial \bar{\partial} \varphi_{m}
$$

extends to a closed positive current on $X$ by a local argument. This completes the proof of the lemma.

Lemma 4.9. Let $\Omega^{\dagger}$ be a smooth volume form on $X^{\dagger}$. Then there exists a divisor $D$ on $X^{\dagger}$ and a constant $C>0$ such that

$$
e^{\varphi_{m}} \Omega_{m} \leq\left|S_{D}\right|_{h_{D}}^{-2} \Omega^{\dagger},
$$

where $S_{D}$ is a defining section of $[D]$ and $h_{D}$ is a fixed smooth Hermitian metric of the line bundle associated to $[D]$.

Proof. We consider again the following commutative diagram:

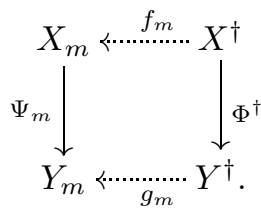

Let $\omega$ be a Kähler metric and $\Omega^{\dagger}$ be a smooth and nowhere-vanishing volume form on $X^{\dagger}$. From the commutative diagram, on a Zariski open set of $X^{\dagger}$ we have 


$$
\left(\sqrt{-1} \partial \bar{\partial} \log \Omega_{m}+\sqrt{-1} \partial \bar{\partial} \varphi_{m}\right)^{\kappa} \wedge \omega^{n-\kappa}=e^{\varphi_{m}}\left(\Phi^{\dagger}\right)^{*}\left(\Phi^{\dagger}\right)_{*} \Omega_{m} \wedge \omega^{n-\kappa} .
$$

Let $\psi_{m}=\varphi_{m}+\log \frac{\Omega_{m}}{\Omega}, \chi_{0}=\sqrt{-1} \partial \bar{\partial} \log \Omega_{0}$.

$\psi_{m}$ satisfies the following Monge-Ampère equation on $X^{\dagger} \backslash\left(\pi^{\dagger}\right)^{-1} B s\left(\left|m_{0} ! K_{X}\right|\right)$,

$$
\left(\chi_{0}+\sqrt{-1} \partial \bar{\partial} \psi_{m}\right)^{\kappa} \wedge \omega^{n-\kappa}=F e^{\psi_{m}} \Omega^{\dagger},
$$

where

$$
F=\frac{\left(\Phi^{\dagger}\right)^{*}\left(\Phi^{\dagger}\right)_{*} \Omega_{m} \wedge \omega^{n-\kappa}}{\Omega_{m}}=\frac{\left(\Phi^{\dagger}\right)^{*}\left(\Phi^{\dagger}\right)_{*} \Omega_{m_{0}} \wedge \omega^{n-\kappa}}{\Omega_{m_{0}}} .
$$

Let $D_{1}$ be a divisor of $X^{\dagger}$ containing $\left(\pi^{\dagger}\right)^{-1} B s\left(\left|m_{0} ! K_{X}\right|\right)$ such that the defining section $S_{1}$ of $\left[D_{1}\right]$ satisfies

$$
\left|S_{1}\right|_{h_{1}}^{2} \frac{\omega^{n}}{F \Omega^{\dagger}}<\infty
$$

where $h_{1}$ is a fixed smooth Hermitian metric of the line bundle associated to $\left[D_{1}\right]$. Obviously, $D_{1}$ is independent of the choice of $m$. Let $\Theta_{h_{1}}=-\sqrt{-1} \partial \bar{\partial} \log h_{1}$.

Let $D_{2}$ be an ample divisor of $X^{\dagger}$ independent on the choice of $m$ such that for a smooth Hermitian metric $h_{2}$, we have

$$
\Theta_{h_{2}}=-\sqrt{-1} \partial \bar{\partial} \log h_{2}>-\Theta_{h_{1}} .
$$

Let $S_{2}$ be the defining section of $D_{2}$.

Let $D_{3}$ be a divisor of $X^{\dagger}$ depending on the choice of $m$ such that $\psi_{m} \in$ $C^{\infty}\left(X^{\dagger} \backslash D_{3}\right)$. Let $S_{3}$ be the defining section of $D_{3}$ and $h_{3}$ be a fixed smooth Hermitian metric of the line bundle associated to $\left[D_{3}\right]$. We define $\Theta_{h_{3}}=-\sqrt{-1} \partial \bar{\partial} \log h_{3}$ and for $\epsilon>0$ sufficiently small,

$$
\Theta_{h_{2}}>-\Theta_{h_{1}}-\epsilon \Theta_{h_{3}} .
$$

Now we let

$$
\psi_{m, \epsilon}=\varphi_{m}+\log \left|S_{1}\right|_{h_{1}}^{2}+\log \left|S_{2}\right|_{h_{2}}^{2}+\epsilon \log \left|S_{3}\right|_{h_{3}}^{2},
$$

and so $\psi_{m, \epsilon}$ satisfies

$$
\left(\chi_{0}+\Theta_{h_{1}}+\Theta_{h_{2}}+\epsilon \Theta_{h_{3}}+\sqrt{-1} \partial \bar{\partial} \log \psi_{m, \epsilon}\right)^{\kappa} \wedge \omega^{n-\kappa}=\frac{F e^{\psi_{m, \epsilon}}}{\left|S_{1}\right|_{h_{2}}^{2}\left|S_{2}\right|_{h_{2}}^{2}\left(\left|S_{3}\right|_{h_{3}}^{2}\right)^{\epsilon}} \Omega^{\dagger} .
$$

The maximum of $\psi_{m, \epsilon}$ can only be achieved in $X^{\dagger} \backslash\left(D_{1} \cup D_{2} \cup D_{3}\right)$. Then by the maximum principle,

$$
\sup _{X^{\dagger}} \psi_{m, \epsilon} \leq \sup _{X^{\dagger}}\left(\left|S_{1}\right|_{h_{1}}^{2}\left|S_{2}\right|_{h_{2}}^{2}\left(\left|S_{3}\right|_{h_{3}}^{2}\right)^{\epsilon} \frac{\left(\chi_{0}+\Theta_{h_{1}}+\Theta_{h_{2}}+\epsilon \Theta_{h_{3}}\right)^{\kappa} \wedge \omega^{n-\kappa}}{F \Omega^{\dagger}}\right)=C_{m, \epsilon}
$$

where $\lim _{\epsilon \rightarrow 0} C_{m, \epsilon}=C_{m, 0}=\sup _{X^{\dagger}}\left(\left|S_{1}\right|_{h_{1}}^{2}\left|S_{2}\right|_{h_{2}}^{2} \frac{\left(\chi_{0}+\Theta_{h_{1}}+\Theta_{h_{2}}\right)^{\kappa} \wedge \omega^{n-\kappa}}{F \Omega^{\dagger}}\right)$ and $C_{m, 0}$ is independent of the choice of $m$.

Now let $\epsilon$ tend to 0 . Then there exists a constant $C>0$ independent of the choice of $m$ such that

$$
\sup _{X^{\dagger}} \psi_{m} \leq C ;
$$

that is, there exists $C^{\prime}>0$ independent of $m$ such that

$$
e^{\varphi_{m}} \Omega_{m} \leq C^{\prime}\left|S_{1}\right|_{h_{1}}^{-2}\left|S_{2}\right|_{h_{2}}^{-2} \Omega^{\dagger} .
$$


Proposition 4.8. There exists a measure $\Omega_{\text {can }}$ on $X$ such that:

(1) $\Omega_{\text {can }}=\lim _{m \rightarrow \infty} e^{\varphi_{m}} \Omega_{m}$.

(2) $\left(K_{X}, \Omega_{c a n}^{-1}\right)$ is an analytic Zariski decomposition. Furthermore,

$$
\frac{\Omega_{\text {can }}}{\Omega_{0}}<\infty \text { and } \frac{\Psi_{X, \epsilon}}{\Omega_{\text {can }}}<\infty,
$$

where $\Omega_{0}$ is a fixed smooth volume form on $X$ and $\Psi_{X, \epsilon}$ is defined as in (1.7).

Proof. Let $\chi_{0}=\operatorname{Ric}\left(\Omega_{0}\right)$. Then $\varphi_{m}+\log \frac{\Omega_{m}}{\Omega_{0}} \in \operatorname{PSH}\left(X, \chi_{0}\right)$. By Corollary 4.3. $\varphi_{m}+\log \frac{\Omega_{m}}{\Omega_{0}}$ is uniformly bounded in $L^{1}(X)$ and so $\varphi_{m}+\log \frac{\Omega_{m}}{\Omega_{0}}$ is uniformly bounded above since $\varphi_{m}+\log \frac{\Omega_{m}}{\Omega_{0}} \in P S H\left(X, \chi_{0}\right)$. By Lemma 4.9, we can define $\Omega_{\text {can }}$ by

$$
\Omega_{c a n}=\lim _{m \rightarrow \infty} e^{\varphi_{m}} \Omega_{m}
$$

and so $\frac{\Omega_{\text {can }}}{\Omega_{0}}<\infty$. Since $\varphi_{m}+\log \frac{\Omega_{m}}{\Omega_{0}} \in P S H\left(X, \chi_{0}\right),\left\{\varphi_{m}+\log \frac{\Omega_{m}}{\Omega_{0}}\right\}_{m=m_{0}}^{\infty}$ is convergent in $L^{1}(X)$. Also $P S H\left(X, \chi_{0}\right) \cap L^{1}(X)$ is closed in $L^{1}(X)$. Therefore

$$
\lim _{m \rightarrow \infty}\left(\varphi_{m}+\log \frac{\Omega_{m}}{\Omega_{0}}\right)=\log \frac{\Omega_{c a n}}{\Omega_{0}}
$$

in $P S H\left(X, \chi_{0}\right) \cap L^{1}(X)$ and $\log \frac{\Omega_{c a n}}{\Omega_{0}}<\infty$.

Let $h_{c a n}=\Omega_{c a n}^{-1}$ be the Hermitian metric on $K_{X}$. By the construction of $\Omega_{m}$,

$$
|\sigma|_{h_{c a n}^{m}}^{2}<\infty
$$

for any section $\sigma \in H^{0}\left(X, m K_{X}\right)$. Hence $\frac{\Psi_{X, \epsilon}}{\Omega_{c a n}}<\infty$ and

$$
H^{0}\left(X, \mathcal{O}_{X}\left(m K_{X}\right) \otimes \mathcal{I}\left(h_{c a n}^{m}\right)\right) \rightarrow H^{0}\left(X, \mathcal{O}_{X}\left(m K_{X}\right)\right)
$$

is an isomorphism and $\left(K_{X}, h_{c a n}\right)$ is an analytic Zariski decomposition.

Proposition 4.9. Let $\Omega^{\dagger}=\left(\pi^{\dagger}\right)^{*} \Omega_{\text {can }}$. There exists a closed positive $(1,1)$-current $\omega^{\dagger}$ on $Y^{\dagger}$ such that $\left(\Phi^{\dagger}\right)^{*} \omega^{\dagger}=\sqrt{-1} \partial \bar{\partial} \log \Omega^{\dagger}$ on a Zariski open set of $X^{\dagger}$. Furthermore, on a Zariski open set of $Y^{\dagger}$, we have

$$
\left(\omega^{\dagger}\right)^{\kappa}=\left(\Phi^{\dagger}\right)_{*} \Omega^{\dagger}
$$

and so

$$
\operatorname{Ric}\left(\omega^{\dagger}\right)=-\omega^{\dagger}+\omega_{W P} .
$$

Proof. Let $\psi_{m}=\varphi_{m}+\log \frac{\Omega_{m}}{\Omega_{m}}$. Both $\psi_{m}$ and $\varphi_{m}$ descend to $Y^{\dagger}$ and by Proposition 4.8

$$
\lim _{m \rightarrow \infty} \psi_{m}=\psi_{\infty}=\log \frac{\Omega^{\dagger}}{\Omega_{m_{0}}} .
$$

Consider

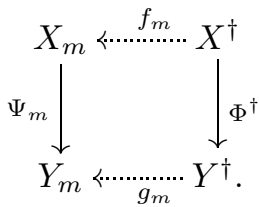


For simplicity, we use $\omega_{m}$ for $\left(g_{m}\right)^{*} \omega_{m}$. Let $D_{m_{0}}$ be a divisor of $Y^{\dagger}$ such that on $X \backslash D_{m_{0}}, \omega_{0}$ is smooth and $\log \frac{\Omega^{\dagger}}{\Omega_{m_{0}}}<\infty$. Also on $Y \backslash D_{m_{0}}$,

$$
\left(\omega_{m_{0}}+\sqrt{-1} \partial \bar{\partial} \psi_{m}\right)^{\kappa}=e^{\varphi_{m}}\left(\Phi^{\dagger}\right)_{*} \Omega_{m} .
$$

Since $\psi_{m}$ converges uniformly on any compact set of $Y^{\dagger} \backslash D_{m_{0}}$ to $\psi_{\infty}$, we have on $Y^{\dagger} \backslash D_{m_{0}}$,

$$
\begin{aligned}
\left(\omega_{m_{0}}+\sqrt{-1} \partial \bar{\partial} \psi_{\infty}\right)^{\kappa} & =\lim _{m \rightarrow \infty}\left(\omega_{m_{0}}+\sqrt{-1} \partial \bar{\partial} \log \psi_{m}\right)^{n} \\
& =\lim _{m \rightarrow \infty} e^{\varphi_{m}}\left(\Phi^{\dagger}\right)_{*} \Omega_{m}=\left(\Phi^{\dagger}\right)_{*} \Omega^{\dagger}
\end{aligned}
$$

Let $\omega^{\dagger}=\omega_{m_{0}}+\sqrt{-1} \partial \bar{\partial} \psi_{\infty}$. Since it is a closed positive current on $Y^{\dagger} \backslash D_{m_{0}}$, it can be extended to a closed positive current on $Y^{\dagger}$.

Also $\left(\Phi^{\dagger}\right)^{*} \omega_{m_{0}}=\sqrt{-1} \partial \bar{\partial} \log \Omega_{m_{0}}$ on $X^{\dagger} \backslash\left(\pi^{\dagger}\right)^{-1}\left(B s\left(\left|m_{0} ! K_{X}\right|\right)\right)$. This implies that on $X^{\dagger} \backslash\left(\pi^{\dagger}\right)^{-1}\left(B s\left(\left|m_{0} ! K_{X}\right|\right)\right)$,

$$
\left(\Phi^{\dagger}\right)^{*} \omega^{\dagger}=\sqrt{-1} \partial \bar{\partial} \log \Omega^{\dagger} .
$$

Furthermore, we have $\frac{\Omega_{m_{0}}}{\Omega^{\dagger}}=\frac{\left(\Phi^{\dagger}\right)_{*} \Omega_{m_{0}}}{\left(\Phi^{\dagger}\right)_{*} \Omega^{\dagger}}$ and so

$$
\begin{aligned}
\operatorname{Ric}\left(\omega^{\dagger}\right) & =\sqrt{-1} \partial \bar{\partial} \log \left(\Phi^{\dagger}\right)_{*} \Omega^{\dagger} \\
& =-\omega^{\dagger}+\sqrt{-1} \partial \bar{\partial} \log \left(\Phi^{\dagger}\right)_{*} \Omega_{m_{0}}-\sqrt{-1} \partial \bar{\partial} \log \Omega_{m_{0}} \\
& =-\omega^{\dagger}+\omega_{W P .}
\end{aligned}
$$

Proposition 4.8 and Proposition 4.9 conclude the proof of Theorem B.2.

4.5. Uniqueness assuming finite generation of the canonical ring. If the canonical ring $\left(X, R_{X}\right)$ is finitely generated, the canonical model $X_{\text {can }}$ is unique and can be constructed by the pluricanonical system $\left|m K_{X}\right|$ for sufficiently large $m$. In this section, we will prove the uniqueness of the canonical measures constructed in Sections 4.3 and 4.4 by assuming a finite generation of the canonical ring. Furthermore, the canonical measure can be considered as a birational invariant.

Theorem 4.2. Let $X$ be a projective manifold of general type. If the canonical ring $R\left(X, K_{X}\right)$ is finitely generated, the Kähler-Einstein measure in Theorem B.1 is constructed in finite steps. Furthermore, it is continuous on $X$ and smooth on a Zariski open dense set of $X$.

Theorem 4.2 is an immediate consequence from the proof of Theorem B.1 with the assumption of a finite generation of the canonical ring. The following theorem is proved in EyGuZe1.

Theorem 4.3. Let $X$ be a projective manifold of general type. If the canonical ring $R\left(X, K_{X}\right)$ is finitely generated, $X_{\text {can }}$ will have only canonical singularities and there exists a unique Kähler-Einstein metric $\omega_{\text {can }}$ on $X_{\text {can }}$ with a continuous potential.

Theorem C.1 is then proved as a corollary of Theorem 4.2 and Theorem 4.3

Corollary 4.4. Let $X$ be an $n$-dimensional projective manifold of general type. Suppose that the canonical ring $R\left(X, K_{X}\right)$ is finitely generated and $\pi: X \rightarrow X_{\text {can }}$ is the pluricanonical map. Let $\omega_{\text {can }}$ be the unique Kähler-Einstein metric on $X_{\text {can }}$ as in Theorem 4.3 and $\Omega_{K E}=\pi^{*}\left(\omega_{\text {can }}^{n}\right)$. Then $\left(X, \Omega_{K E}^{-1}\right)$ coincides with the analytic Zariski decomposition constructed in Theorem B.1. 
Proof. Since the canonical ring $R\left(X, K_{X}\right)$ is finitely generated, the pluricanonical map is stabilized for sufficiently large powers so that the proof of Theorem B.1 terminates in a finite number of steps. It is then straightforward to check that the Kähler-Einstein metric constructed in Theorem B.1 satisfies the same Monge-Ampère equation on the unique canonical model of $X$ in Theorem 4.3 (see EyGuZe1).

We shall now prove Theorem C.2.

Definition 4.2. Suppose that $X$ is an $n$-dimensional projective manifold of Kodaira dimension $0<\kappa<n$ and the canonical ring $R\left(X, K_{X}\right)$ is finitely generated. Let $\Phi: X \rightarrow X_{\text {can }}$ be the pluricanonical map. There exists a nonsingular model $X^{\dagger}$ of $X$ and the following diagram holds:

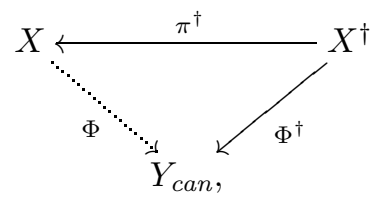

where $\pi^{\dagger}$ is birational and the generic fibre of $\Phi^{\dagger}$ has Kodaira dimension 0.

(1) Then the pushforward measure $\Phi_{*} \Omega$ on $X_{\text {can }}$ is defined by

$$
\Phi_{*} \Omega=\left(\Phi^{\dagger}\right)_{*}\left(\left(\pi^{\dagger}\right)^{*} \Omega\right) .
$$

(2) Let $\Phi=\Phi_{m}$ be the pluricanonical map associated to a basis $\left\{\sigma_{j_{m}}\right\}_{j_{m}=0}^{d_{m}}$ of the linear system $\left|m K_{X}\right|$, for $m$ sufficiently large. Also let $\Omega_{m}=$ $\left(\sum_{j_{m}=0}^{d_{m}} \sigma_{j_{m}} \otimes \overline{\sigma_{j_{m}}}\right)^{\frac{1}{m}}$ and $\omega_{F S}$ be the Fubini-Study metric of $\mathbf{C P}^{d_{m}}$ restricted on $X_{c a n}$ associated to $\Phi_{m}$. Then we defined $\bar{\omega}_{W P}$ by

$$
\bar{\omega}_{W P}=\frac{1}{m} \omega_{F S}+\sqrt{-1} \partial \bar{\partial} \log \Phi_{*} \Omega_{m} .
$$

In particular, $\bar{\omega}_{W P}$ coincides with $\omega_{W P}$ in Definition 4.1 on a Zariski open set of $X_{\text {can }}$.

Lemma 4.10. $\Phi_{*} \Omega$ is independent of the choice of the diagram in Definition 4.2 .

Proof. Let $\rho$ be a test function on $X_{c a n}$. Then

$$
\int_{X_{c a n}} \rho \Phi_{*} \Omega=\int_{X^{\dagger}}\left(\left(\Phi^{\dagger}\right)^{*} \rho\right)\left(\pi^{\dagger}\right)^{*} \Omega=\int_{X}\left(\Phi^{*} \rho\right) \Omega,
$$

which is independent of the choice of the diagram (4.53).

Since the generic fibre of $\Phi^{\dagger}$ has Kodaira dimension 0, by the same argument as in Lemma 4.5, we have the following lemma.

Lemma 4.11. Let $\left\{\sigma_{j_{p}}^{(1)}\right\}_{j_{p}=0}^{d_{p}}$ and $\left\{\sigma_{j_{q}}^{(2)}\right\}_{j_{q}=0}^{d_{q}}$ be bases of the linear systems $\left|p K_{X}\right|$ and $\left|q K_{X}\right|$, for $p$ and $q$ sufficiently large. Let $\Omega^{(1)}=\left(\sum_{j_{p}=0}^{d_{p}} \sigma_{j_{p}} \otimes \overline{\sigma_{j_{p}}}\right)^{\frac{1}{p}}$ and $\Omega^{(2)}=\left(\sum_{j_{q}=0}^{d_{q}} \sigma_{j_{q}} \otimes \overline{\sigma_{j_{q}}}\right)^{\frac{1}{q}}$. Then $\left(\pi^{\dagger}\right)^{*}\left(\frac{\Omega^{(1)}}{\Omega^{(2)}}\right)$ is constant on any generic fibre and so

$$
\left(\pi^{\dagger}\right)^{*}\left(\frac{\Omega^{(1)}}{\Omega^{(2)}}\right)=\left(\Phi^{\dagger}\right)^{*}\left(\frac{\Phi_{*} \Omega^{(1)}}{\Phi_{*} \Omega^{(2)}}\right) .
$$


Lemma 4.12. The definition of $\bar{\omega}_{W P}$ only depends on $X$.

Proof. By Lemma 4.10, the definition of $\bar{\omega}_{W P}$ does not depend on the choice of the diagram (4.53). Let $\left\{\sigma_{j_{p}}^{(1)}\right\}_{j_{p}=0}^{d_{p}}$ and $\left\{\sigma_{j_{q}}^{(2)}\right\}_{j_{q}=0}^{d_{q}}$ be bases of the linear systems $\left|p K_{X}\right|$ and $\left|q K_{X}\right|$, for $p$ and $q$ sufficiently large. Let $\Omega^{(1)}=\left(\sum_{j_{p}=0}^{d_{p}} \sigma_{j_{p}} \otimes \overline{\sigma_{j_{p}}}\right)^{\frac{1}{p}}$ and $\Omega^{(2)}=\left(\sum_{j_{q}=0}^{d_{q}} \sigma_{j_{q}} \otimes \overline{\sigma_{j_{q}}}\right)^{\frac{1}{q}}$.

Let $\omega_{F S}^{(1)}$ and $\omega_{F S}^{(2)}$ be the Fubini-Study metrics of $\mathbf{C P}{ }^{d_{p}}$ and $\mathbf{C P}^{d_{q}}$ restricted on $X_{\text {can }}$ associated to $\Phi_{p}$ and $\Phi_{q}$. Then by avoiding the base locus of $R\left(X^{\dagger}, K_{X^{\dagger}}\right)$, there exist a Zariski open set $U$ of $X_{\text {can }}$ and a Zariski open set $V$ of $X^{\dagger}$, such that on $V$,

$$
\frac{1}{p}\left(\Phi^{\dagger}\right)^{*} \omega_{F S}^{(1)}=\sqrt{-1} \partial \bar{\partial} \log \left(\pi^{\dagger}\right)^{*} \Omega^{(1)}, \frac{1}{q}\left(\Phi^{\dagger}\right)^{*} \omega_{F S}^{(2)}=\sqrt{-1} \partial \bar{\partial} \log \left(\pi^{\dagger}\right)^{*} \Omega^{(2)}
$$

and so on $U$,

$$
\frac{1}{p} \omega_{F S}^{(1)}-\frac{1}{q} \omega_{F S}^{(2)}=\sqrt{-1} \partial \bar{\partial} \log \left(\frac{\Phi_{*} \Omega^{(1)}}{\Phi_{*} \Omega^{(2)}}\right) .
$$

Since $\frac{1}{p} \omega_{F S}^{(1)}$ and $\frac{1}{q} \omega_{F S}^{(2)}$ are in the same class, and $\log \left(\frac{\Phi_{*} \Omega^{(1)}}{\Phi_{*} \Omega^{(2)}}\right)$ is in $L^{\infty}\left(X_{c a n}\right)$, equation (4.57) holds everywhere on $X_{\text {can }}$. Therefore the following equality completes the proof of the lemma:

$$
\frac{1}{p} \omega_{F S}^{(1)}-\sqrt{-1} \partial \bar{\partial} \log \Phi_{*} \Omega^{(1)}=\frac{1}{q} \omega_{F S}^{(2)}-\sqrt{-1} \partial \bar{\partial} \log \Phi_{*} \Omega^{(2)} .
$$

Theorem 4.4. Suppose that $X$ is an $n$-dimensional projective manifold of Kodaira dimension $0<\kappa<n$. If the canonical ring $R\left(X, K_{X}\right)$ is finitely generated and $X_{\text {can }}$ is the canonical model of $X$, then there exists a unique canonical measure $\Omega_{\text {can }}$ on $X$ satisfying:

(1) $\Omega_{\text {can }}$ is continuous on $X$ and smooth on a Zariski open set of $X$.

(2) $0<\frac{\Omega_{c a n}}{\Psi_{X}}<\infty$ and $\left(K_{X}, \Omega_{c a n}^{-1}\right)$ is an analytic Zariski decomposition.

(3) Let $\Phi: X \rightarrow X_{\text {can }}$ be the pluricanonical map. Then there exists a unique closed positive $(1,1)$-current $\omega_{\text {can }}$ with continuous local potentials on $X_{\text {can }}$ such that $\Phi^{*} \omega_{\text {can }}=\sqrt{-1} \partial \bar{\partial} \log \Omega_{\text {can }}$ outside the base locus of the pluricanonical system. Furthermore,

$$
\left(\omega_{c a n}\right)^{\kappa}=\Phi_{*} \Omega_{c a n},
$$

so on a Zariski open set of $X_{\text {can }}$ we have

$$
\operatorname{Ric}\left(\omega_{c a n}\right)=-\omega_{c a n}+\bar{\omega}_{W P} .
$$

Furthermore, $\Omega_{\text {can }}$ is invariant under birational transformations.

Proof. If $R\left(X, K_{X}\right)$ is finitely generated, there exists the following diagram:

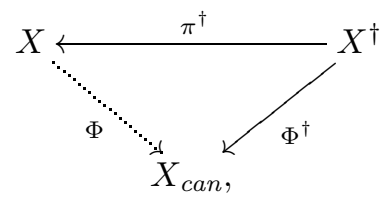


where $X_{\text {can }}$ is the canonical model of $X$, and $X^{\dagger}$ is the resolution of the stable base locus of the pluricanonical systems such that $\left(\pi^{\dagger}\right)^{*} M K_{X}=L_{M}+E_{M}$ for sufficiently large $M$, where $L_{M}$ is globally generated and $E_{M}$ is the fixed part of $\left|\left(\pi^{\dagger}\right)^{*} M K_{X}\right|$ with $E_{M}$ being a divisor with normal crossings. $X^{\dagger}$ is an Iitaka fibration over $X_{c a n}$ such that the generic fibre has Kodaira dimension 0. Let $\left\{\sigma_{j}\right\}_{j=0}^{d_{M}}$ be a basis of $H^{0}\left(X, M K_{X}\right)$ and $\left\{\zeta_{j}\right\}_{j=0}^{d_{M}}$ be a basis of $H^{0}\left(X, L_{M}\right)$ such that

$$
\left(\pi^{\dagger}\right)^{*} \sigma_{j}=\zeta_{j} E_{M}
$$

Let $\Omega=\pi^{\dagger}\left(\sum_{m=0}^{M} \sum_{j_{m}=0}^{d_{m}}\left|\sigma_{j_{m}}\right|^{\frac{2}{m}}\right)$ be a degenerate smooth volume form on $X^{\dagger}$ and $\omega=\frac{1}{M} \sqrt{-1} \partial \bar{\partial} \log \left(\sum_{j=0}^{d_{M}}\left|\zeta_{j}\right|^{2}\right)$. Then the following Monge-Ampère equation has a unique continuous solution $\varphi$ on $X_{c a n}$,

$$
(\omega+\sqrt{-1} \partial \bar{\partial} \varphi)^{\kappa}=e^{\varphi}\left(\Phi^{\dagger}\right)_{*} \Omega .
$$

Furthermore, $\varphi$ is smooth on a Zariski open set $X_{c a n}^{\circ}$ of $X_{\text {can }}$ and so is $\omega_{\text {can }}=$ $\omega+\sqrt{-1} \partial \bar{\partial} \varphi$.

Let $\Theta=\frac{\Omega}{\left(\Phi^{\dagger}\right)^{*}\left(\Phi^{\dagger}\right)_{*} \Omega}$ be an $(n-\kappa, n-\kappa)$-current on $X^{\dagger}$. On a generic fibre $F$, $\left.\Theta\right|_{F}=\eta \wedge \bar{\eta}$ for some $\eta \in H^{0}\left(X, K_{F}\right)$. So without loss of generality, we assume that on $X_{\text {can }}^{\circ}$,

$$
\omega_{W P}=\sqrt{-1} \partial \bar{\partial} \log \Theta-\frac{1}{M} \sqrt{-1} \partial \bar{\partial} \log \left|E_{M}\right|^{2} .
$$

Therefore on $X_{c a n}^{\circ}$,

$$
\operatorname{Ric}\left(\omega_{c a n}\right)=-\omega_{c a n}+\bar{\omega}_{W P} .
$$

On the other hand, we define

$$
\Omega_{c a n}=\left(\left(\pi^{\dagger}\right)^{-1}\right)^{*}\left(e^{\varphi} \Omega\right)=e^{\Phi^{*} \varphi}\left(\sum_{m=0}^{M} \sum_{j_{m}=0}^{d_{m}}\left|\sigma_{j_{m}}\right|^{\frac{2}{m}}\right) .
$$

From the regularity of $\varphi, \Omega_{c a n}$ is continuous on $X$ and smooth on a Zariski open set of $X$ and

$$
\sqrt{-1} \partial \bar{\partial} \log \Omega_{c a n}=\sqrt{-1} \partial \bar{\partial} \log \left(\sum_{m=0}^{M} \sum_{j_{m}=0}^{d_{m}}\left|\sigma_{j_{m}}\right|^{\frac{2}{m}}\right)+\sqrt{-1} \partial \bar{\partial} \Phi^{*} \varphi=\Phi^{*} \omega_{c a n} .
$$

We then shall prove the uniqueness of $\Omega_{c a n}$. Suppose there exists another measure $\Omega^{\prime}$ satisfying the assumptions in the theorem. Then let $\Omega^{\prime}=e^{\varphi^{\prime}} \Omega$. Since $\Phi^{*} \omega_{F S}=\sqrt{-1} \partial \bar{\partial} \log \Omega, \sqrt{-1} \partial \bar{\partial} \log \Omega^{\prime}-\sqrt{-1} \partial \bar{\partial} \log \Omega$ is a pullback from $X_{\text {can }}$. Therefore on a Zariski open set where $F$ is a generic fibre of $\Phi^{\dagger}$, we have

$$
\sqrt{-1} \partial_{F} \bar{\partial}_{F} \log \left(\pi^{\dagger}\right)^{*}\left(\frac{\Omega^{\prime}}{\Omega}=0\right)=\sqrt{-1} \partial_{F} \bar{\partial}_{F}\left(\pi^{\dagger}\right)^{*} \varphi^{\prime}
$$

Since $\left(\pi^{\dagger}\right)^{*} \varphi^{\prime} \in L^{\infty}(F),\left(\pi^{\dagger}\right)^{*} \varphi^{\prime}$ is constant along $F$. So $\varphi^{\prime}$ descends to $X_{c a n}$ and satisfies the following Monge-Ampère equation:

$$
\left(\omega+\sqrt{-1} \partial \bar{\partial} \varphi^{\prime}\right)^{\kappa}=e^{\varphi^{\prime}} \Phi_{*} \Omega .
$$

By the uniqueness of the solution of equation (4.59), $\Omega^{\prime}=\Omega_{c a n}$ and we have proved the uniqueness of $\Omega_{c a n}$. 
Finally we shall prove that $\Omega_{\text {can }}$ and $\Phi^{*} \omega_{\text {can }}$ are birational invariants. Suppose $X_{(1)}$ and $X_{(2)}$ are birational with $X_{c a n}$ being the canonical model. Then we have the following diagram:

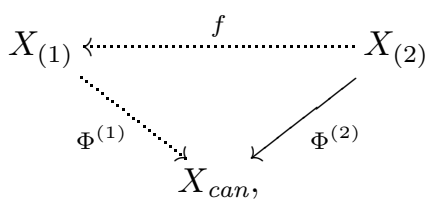

where $f$ is birational and $\Phi^{(1)}$ and $\Phi^{(2)}$ are the pluricanonical maps. Fix $\Omega$ on $X_{(1)}$ as constructed as in the proof of uniqueness. Then by Hartogs' theorem, $f^{*} \Omega$ is smooth and can be constructed the same way. By replacing $X_{(1)}$ and $X_{(2)}$ by their Iitaka fibration, it is straightforward to show that

$$
\left(\Phi^{(1)}\right)_{*} \Omega=\left(\Phi^{(2)}\right)_{*}\left(f^{*} \Omega\right) .
$$

Let $\left(\Phi^{(1)}\right)^{*} \omega=\sqrt{-1} \partial \bar{\partial} \log \Omega, \Omega_{(1)}=e^{\varphi_{(1)}} \Omega$ and $\Omega_{(2)}=e^{\varphi_{(2)}} f^{*} \Omega$ be the unique canonical measures on $X_{(1)}$ and $X_{(2)}$. Both $\varphi_{(1)}$ and $\varphi_{(2)}$ descend to $\operatorname{PSH}(X, \omega) \cap$ $L^{\infty}\left(X_{\text {can }}\right)$ and satisfy

$$
(\omega+\sqrt{-1} \partial \bar{\partial} \varphi)^{\kappa}=e^{\varphi}\left(\Phi^{(1)}\right)_{*} \Omega .
$$

The uniqueness of the solution to the above Monge-Ampère equation implies that $\varphi_{(1)}=\varphi_{(2)}$ and so

$$
f^{*} \Omega_{(1)}=\Omega_{(2)} .
$$

\section{THE KÄHLER-RICCI FLOW}

5.1. Reduction of the normalized Kähler-Ricci flow. Let $X$ be an $n$-dimensional compact Kähler manifold. A Kähler metric can be given by its Kähler form $\omega$ on $X$. In local coordinates $z_{1}, \ldots, z_{n}$, we can write $\omega$ as

$$
\omega=\sqrt{-1} \sum_{i, j=1}^{n} g_{i \bar{j}} d z_{i} \wedge d z_{\bar{j}},
$$

where $\left\{g_{i \bar{j}}\right\}$ is a positive definite Hermitian matrix function. Consider the normalized Kähler-Ricci flow

$$
\left\{\begin{array}{l}
\frac{\partial \omega(t, \cdot)}{\partial t}=-\operatorname{Ric}(\omega(t, \cdot))-\omega(t, \cdot), \\
\omega(0, \cdot)=\omega_{0},
\end{array}\right.
$$

where $\operatorname{Ric}(\omega(t, \cdot))$ denotes the Ricci curvature of $\omega(t, \cdot)$ and $\omega_{0}$ is a given Kähler metric.

Let $K a(X)$ denote the Kähler cone of $X$, that is,

$$
K a(X)=\left\{[\omega] \in H^{1,1}(X, \mathbf{R}) \mid[\omega]>0\right\} .
$$


Suppose that $\omega(t, \cdot)$ is a solution of (5.60) on $[0, T)$. Then its induced equation on Kähler classes in $K a(X)$ is given by the following ordinary differential equation:

$$
\left\{\begin{array}{l}
\frac{\partial[\omega]}{\partial t}=-2 \pi c_{1}(X)-[\omega], \\
{\left.[\omega]\right|_{t=0}=\left[\omega_{0}\right] .}
\end{array}\right.
$$

It follows that

$$
[\omega(t, \cdot)]=-2 \pi c_{1}(X)+e^{-t}\left(\left[\omega_{0}\right]+2 \pi c_{1}(X)\right) .
$$

Now if we assume that the canonical bundle $K_{X}$ is semi-positive, then for a sufficiently large integer $m$, the pluricanonical map associated to $H^{0}\left(X, m K_{X}\right)$ gives rise to an algebraic fibre space $f: X \rightarrow X_{c a n}$, where $X_{c a n}$ is the canonical model of $X$. Recall that the Kodaira dimension $\operatorname{kod}(X)$ of $X$ is defined to be the dimension of $X_{\text {can }}$. Moreover, there is a smooth Kähler form $\chi$ as the Fubini-Study metric associated to a basis of $H^{0}\left(X, m K_{X}\right)$ on the normal Kähler space $X_{\text {can }}$ such that $f^{*} \chi$ represents $-2 \pi c_{1}(X)$. Choose the reference Kähler metric $\omega_{t}$ by

$$
\omega_{t}=\chi+e^{-t}\left(\omega_{0}-\chi\right) \text {. }
$$

Here we abuse the notation by identifying $\chi$ and $f^{*} \chi$ for simplicity. Then the solution of (5.60) can be written as

$$
\omega=\omega_{t}+\sqrt{-1} \partial \bar{\partial} \varphi
$$

We can always choose a smooth volume form $\Omega$ on $X$ such that $\operatorname{Ric}(\Omega)=\chi$. Then the evolution for the Kähler potential $\varphi$ is given by the following initial value problem:

$$
\left\{\begin{array}{l}
\frac{\partial \varphi}{\partial t}=\log \frac{e^{(n-\kappa) t}\left(\omega_{t}+\sqrt{-1} \partial \bar{\partial} \varphi\right)^{n}}{\Omega}-\varphi \\
\left.\varphi\right|_{t=0}=0,
\end{array}\right.
$$

where $\kappa=\operatorname{kod}(X)$.

5.2. Kähler-Ricci flow on projective manifolds with semi-positive canonical line bundle. In this section, we will prove Theorem A.

Theorem 5.1. Let $X$ be an n-dimensional nonsingular projective variety with semi-ample canonical line bundle $K_{X} . X$ admits a holomorphic fibration over its canonical model $X_{\text {can }} f: X \rightarrow X_{\text {can }}$. Suppose $0<\operatorname{dim} X_{\text {can }}=\kappa<n$. Then for any initial Kähler metric, the Kähler-Ricci flow (1.1) has a global solution $\omega(t, \cdot)$ for all time $t \in[0, \infty)$ satisfying:

(1) $\omega(t, \cdot)$ converges to $f^{*} \omega_{\infty} \in-2 \pi c_{1}(X)$ as currents for a closed positive current $\omega_{\text {can }}$ on $X_{\text {can }}$ as in Theorem 3.1 .

(2) $\omega_{\text {can }}$ is smooth on $X_{\text {can }}^{\circ}$ and satisfies the generalized Kähler-Einstein equation on $X_{c a n}^{\circ}$,

$$
\operatorname{Ric}\left(\omega_{c a n}\right)=-\omega_{c a n}+\omega_{W P},
$$

where $\omega_{W P}$ is the induced Weil-Petersson metric.

(3) For any compact subset $K \in X_{\text {can }}^{\circ}$, there is a constant $C_{K}$ such that

$$
\|R(t, \cdot)\|_{L^{\infty}\left(f^{-1}(K)\right)}+e^{(n-\kappa) t} \sup _{s \in K}\left\|\left.(\omega(t, \cdot))^{n-\kappa}\right|_{f^{-1}(s)}\right\|_{L^{\infty}\left(f^{-1}(s)\right)} \leq C_{K} .
$$


Corollary 5.1. Let $X$ be a nonsingular projective variety with semi-ample canonical bundle. If $X_{\text {can }}^{\circ}=X_{\text {can }}$, i.e., $X_{\text {can }}$ is nonsingular and $f: X \rightarrow X_{\text {can }}$ has no singular fibres, then for any initial Kähler metric, the Kähler-Ricci flow (1.1) converges to a smooth limit metric $f^{*} \omega_{\infty} \in K_{X}$ satisfying

$$
\operatorname{Ric}\left(\omega_{\infty}\right)=-\omega_{\infty}+\omega_{W P}
$$

\section{Step 1. Zeroth-order and volume estimates.}

We will first derive the zeroth-order estimates for $\varphi$ and $\frac{\partial \varphi}{\partial t}$.

Lemma 5.1. Let $\varphi$ be a solution of the Kähler-Ricci flow (5.63). There exists a constant $C>0$ such that on $[0, \infty) \times X$,

(1) $\varphi \leq C$,

(2) $\frac{\partial \varphi}{\partial t} \leq C$,

(3) $\frac{e^{(n-\kappa) t} \omega^{n}}{\Omega} \leq C$.

Proof. The lemma is a straightforward application of the maximum principle and can be proved by the same argument as in SoTi].

Proposition 5.1. There exists a constant $C>0$ such that on $[0, \infty) \times X$,

$$
|\varphi| \leq C
$$

Proof. Rewrite the parabolic flow as a family of Monge-Ampère equations

$$
\left(\omega_{t}+\sqrt{-1} \partial \bar{\partial} \varphi\right)^{n}=e^{\frac{\partial \varphi}{\partial t}+\varphi-(n-\kappa) t} \Omega .
$$

We will apply Theorem 2.5 by letting $F(t, \cdot)=e^{\frac{\partial \varphi}{\partial t}+\varphi-(n-\kappa) t}$. Notice that there exists a constant $C_{1}>0$ such that $0<F \leq C_{1} e^{-(n-\kappa) t}$,

$$
\frac{e^{-(n-\kappa) t}}{C_{1}} \leq\left[\omega_{t}\right]^{n} \leq C_{1} e^{-(n-\kappa) t}
$$

and

$$
\frac{e^{-(n-\kappa) t}}{C_{1}} \chi^{\kappa} \wedge \omega_{0}^{n-\kappa} \leq \omega_{t}^{n} \leq C_{1} e^{-(n-\kappa) t} \Omega .
$$

The assumptions in Theorem 2.5 for $F$ and $\omega_{t}$ are satisfied. Therefore $\sup _{X} \varphi-$ $\inf _{X} \varphi$ is uniformly bounded for all $t \in[0, \infty)$. Since $\varphi$ is uniformly bounded from above, the proposition is proved and the uniform $C^{0}$-estimate is obtained.

The following estimate can be proved in the same way as in SoTi].

Lemma 5.2. There exists a divisor $D$ on $X_{\text {can }}$ and constants $C_{1}$ and $C_{2}>0$ such that

$$
\frac{\partial \varphi}{\partial t} \geq C_{1} \log |S|_{h}^{2}-C_{2}
$$

where $S$ is a defining section of $f^{*} D$ and $h$ is a fixed smooth Hermitian metric of the line bundle associated to $\left[f^{*} D\right]$. 
Step 2. Partial second-order estimates and collapsing.

Proposition 5.2. There exist a divisor $D$ on $X_{\text {can }}$ and constants $\lambda, C>0$ such that

$$
\operatorname{tr}_{\omega}(\chi) \leq \frac{C}{|S|_{h}^{2 \lambda}}
$$

where $S$ is a defining section of $f^{*} D$ and $h$ is a smooth Hermitian metric of the line bundle associated to the divisor $\left[f^{*} D\right]$.

Proof. Since $X_{\text {can }}$ might be singular, we can consider the nonsingular model $f^{\prime}$ : $X^{\prime} \rightarrow Y^{\prime}$ for $f: X \rightarrow X_{c a n}$ such that following diagram commutes:

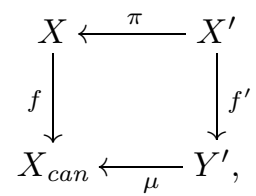

where $\pi$ and $\mu$ are birational.

Let $\varphi^{\prime}=\pi^{*} \varphi, \chi^{\prime}=\mu^{*} \chi, \omega_{0}^{\prime}=\pi^{*} \omega_{0}$ and $\omega^{\prime}=\pi^{*} \omega$. We also write $\chi^{\prime}$ for $\left(f^{\prime}\right)^{*} \chi^{\prime}$ for simplicity.

Let $\theta$ be a Kähler form on $Y^{\prime}$ such that $\theta \geq \chi^{\prime}$. For simplicity, we identify $\theta$ and $\chi^{\prime}$ with $\left(f^{\prime}\right)^{*} \theta$ and $\left(f^{\prime}\right)^{*} \chi^{\prime}$. Since $\chi^{\prime}$ is semi-positive induced by the Fubini-Study metric and it only vanishes along a subvariety of $Y^{\prime}$ with finite order, there exists a divisor $D_{1}$ on $Y^{\prime}$ such that

$$
\theta \leq \frac{1}{\left|S_{1}\right|_{h_{1}}^{2}} \chi^{\prime}
$$

where $S_{1}$ is a defining section of $\left(f^{\prime}\right)^{*} D_{1}$ with $h_{1}$ a smooth Hermitian metric of the line bundle associated to $\left[\left(f^{\prime}\right)^{*} D_{1}\right]$. Without loss of generality, we can assume that the support of $\mu^{*} D$ in Lemma 5.2 is contained in $D_{1}$.

Let

$$
u=\operatorname{tr}_{g^{\prime}}(\theta)=\left(g^{\prime}\right)^{i \bar{j}} \theta_{i \bar{j}},
$$

where $g^{\prime}$ is the Kähler metric associated to $\omega^{\prime}$. The Kähler-Ricci flow for $\omega$ can be pulled back to the Kähler-Ricci flow for $\omega^{\prime}$ on $X^{\prime}$ outside the exceptional divisors. Let $\Delta^{\prime}$ be the Laplace operator associated to $g^{\prime}$. We have then

$$
\operatorname{tr}_{g^{\prime}}(\theta) \leq \frac{1}{\left|S_{1}\right|_{h_{1}}^{2}} \operatorname{tr}_{g^{\prime}}\left(\chi^{\prime}\right) .
$$

Following the similar calculation in SoTi, we have

$$
\left(\frac{\partial}{\partial t}-\Delta^{\prime}\right) \log u \leq C(u+1) .
$$

Since $\left[\chi^{\prime}\right]$ is big and semi-ample, there exists a divisor $D_{2}$ on $Y^{\prime}$ such that $\left[\chi^{\prime}\right]-\epsilon\left[D_{2}\right]$ is ample for any $\epsilon>0$. Then let $S_{2}$ be the defining section for $\left(f^{\prime}\right)^{*} D_{2}$ and there exists a smooth Hermitian metric $h_{2}$ on the line bundle associated to $\left[D_{2}\right]$ such that

$$
\chi^{\prime}-\epsilon \Theta_{h_{2}}=\chi^{\prime}+\epsilon \sqrt{-1} \partial \bar{\partial} \log h_{2}>0 .
$$

For simplicity, we identify $h_{2}$ and $\left(f^{\prime}\right)^{*} h_{2}$. 
Let $D_{3}$ be a divisor on $X^{\prime}$ containing the exceptional divisor of $\pi$ on $X^{\prime}$. Let $S_{3}$ be the defining section of $\left[D_{3}\right]$. There exists a smooth Hermitian metric $h_{3}$ on the line bundle associated to $\left[D_{3}\right]$ such that for all sufficiently small $\delta>0$,

$$
\omega_{0}^{\prime}+\delta \Theta_{h_{3}} \geq 0 \text {. }
$$

We define

$$
\varphi_{\epsilon}^{\prime}=\varphi^{\prime}-\epsilon \log \left|S_{2}\right|_{h_{2}}^{2} .
$$

Then there exists a constant $C>0$ depending on $\epsilon$ such that

$$
\Delta^{\prime} \varphi_{\epsilon}^{\prime}=n-\operatorname{tr}_{g^{\prime}}\left(\omega_{t}^{\prime}-\epsilon \Theta_{h_{2}}\right) \leq n-C \operatorname{tr}_{g^{\prime}} \theta-e^{-t} \operatorname{tr}_{g^{\prime}}\left(\omega_{0}^{\prime}\right)=n-C u-e^{-t} \operatorname{tr}_{g^{\prime}}\left(\omega_{0}^{\prime}\right) .
$$

Calculate for sufficiently large $A>0$ and small $\epsilon>0$ and $\delta>0$,

$$
\begin{array}{r}
\left(\frac{\partial}{\partial t}-\Delta^{\prime}\right)\left(\log \left(\left|S_{1}\right|_{h_{1}}^{4}\left|S_{3}\right|_{h_{3}}^{\left.2 \delta e^{-t} u\right)-2 A \varphi_{\epsilon}^{\prime}}\right)\right. \\
\leq-A u+C-\delta e^{-t} \log \left|S_{3}\right|_{h_{3}}^{2}-2 A \frac{\partial \varphi^{\prime}}{\partial t}
\end{array}
$$

for all $t>0$ in $X^{\prime} \backslash\left(D_{1} \cup D_{2} \cup D_{3}\right)$.

The maximum of $\log \left(\left|S_{1}\right|_{h_{1}}^{4}\left|S_{3}\right|_{h_{3}}^{2 \delta e^{-t}} u\right)-2 A \varphi_{\epsilon}^{\prime}$ can only be achieved on $X^{\prime} \backslash\left(D_{1} \cup\right.$ $\left.D_{2} \cup D_{3}\right)$. The maximum principle implies that there exist constants $\lambda, C>0$ independent of $\delta$ such that for all $(t, z) \in[0, \infty) \times X^{\prime}$,

$$
u(t, z) \leq C\left(\left|S_{1}\right|_{h_{1}}^{-2 \lambda}\left|S_{2}\right|_{h_{2}}^{-2 \lambda}\left|S_{3}\right|_{h_{3}}^{-2 \delta e^{-t}}\right)(z) .
$$

The proposition is then proved by letting $\delta \rightarrow 0$.

From the uniform upper bound of $\omega^{n}$, one immediately concludes that the volume of a regular fibre of $f$ tends to 0 exponentially fast uniformly away from the singular fibres.

Corollary 5.2. There exists a divisor $D$ on $X_{\text {can }}$ and constants $\lambda, C>0$ such that for all $t \geq 0$ and $s \in X_{\text {can }}$,

$$
\frac{\left(\left.\omega\right|_{X_{s}}\right)^{n-\kappa}}{\left(\left.\omega_{0}\right|_{X_{s}}\right)^{n-\kappa}} \leq e^{-(n-\kappa) t} \frac{C}{|S|_{h}^{2}}
$$

where $\left.\omega_{0}\right|_{X_{s}}$ and $\left.\omega\right|_{X_{s}}$ are the restrictions of $\omega_{0}$ and $\omega_{s}$ on $X_{s}=f^{-1}(s), S$ is a defining section of $f^{*} D$ and $h$ is a smooth Hermitian metric of the line bundle associated to the divisor $\left[f^{*} D\right]$.

Proof. Notice that

$\frac{\left(\left.\omega\right|_{X_{s}}\right)^{n-\kappa}}{\left(\left.\omega_{0}\right|_{X_{s}}\right)^{n-\kappa}}=\frac{\omega^{n-\kappa} \wedge \chi^{\kappa}}{\omega_{0}^{n-\kappa} \wedge \chi^{\kappa}}=\frac{\omega^{n-\kappa} \wedge \chi^{\kappa}}{\omega^{n}} \frac{\omega^{n}}{\omega_{0}^{n-\kappa} \wedge \chi^{\kappa}} \leq C\left(\frac{\omega^{n-1} \wedge \chi}{\omega^{n}}\right)^{\kappa} \frac{\omega^{n}}{\omega_{0}^{n-\kappa} \wedge \chi^{\kappa}}$.

The corollary is then proved by Lemma 5.1 and Proposition 5.2 ,

Corollary 5.3. For any compact set $K \subset X_{\text {can }}^{\circ}$, there exists a constant $C_{K}$ such that for all $t \geq 0$ and $s \in K$,

$$
\sup _{X_{s}} \varphi(t, \cdot)-\inf _{X_{s}} \varphi(t, \cdot) \leq C_{K} e^{-t} .
$$

Proof. The Poincaré and Sobolev constants with respect to $\left.\omega_{0}\right|_{X_{s}}$ for $s \in K$ are uniformly bounded. The proof of the corollary is achieved by Corollary 5.2 and Moser's iteration in Yau's $C^{0}$-estimate for the Calabi conjecture. 


\section{Step 3. Gradient estimates.}

The gradient estimates in this section are obtained in the same way as in SoTi], and it is an adaption from the gradient estimate in ChYa and the argument in [Pe] to obtain a uniform bound for $\left|\nabla \frac{\partial \varphi}{\partial t}\right|_{g}$ and the scalar curvature $R$. Let $u=$ $\frac{\partial \varphi}{\partial t}+\varphi=\log \frac{e^{(n-\kappa) t} \omega^{2}}{\Omega}$. The evolution equation for $u$ is given by

$$
\frac{\partial u}{\partial t}=\Delta u+\operatorname{tr}_{\omega}(\chi)-(n-\kappa)
$$

We will obtain a gradient estimate for $u$, which will help us bound the scalar curvature from above. Note that $u$ is uniformly bounded from above, so we can find a constant $A>0$ such that $A-u \geq 1$.

Theorem 5.2. There exist constants $\lambda, C>0$ such that

(1) $|S|_{h}^{2 \lambda}|\nabla u|^{2} \leq C(A-u)$,

(2) $-|S|_{h}^{2 \lambda} \Delta u \leq C(A-u)$,

where $\nabla$ is the gradient operator with respect to the metric $g$ associated to $\omega$ along the flow and $|\cdot|=|\cdot|_{g}$.

Theorem 5.2 is proved the same way as in SoTi] with little modification.

The following corollary is immediate by Theorem [5.2, Lemma 5.1] and Lemma 5.2 .

Corollary 5.4. For any $\delta>0$, there exist constants $\lambda, C>0$ such that

(1) $|S|_{h}^{2 \lambda}|\nabla u|^{2} \leq C$,

(2) $-|S|_{h}^{2 \lambda} \Delta u \leq C$.

Now we are in a position to prove a uniform bound for the scalar curvature. The following corollary tells us that the Kähler-Ricci flow will collapse with bounded scalar curvature away from the singular fibres.

Corollary 5.5. Along the Käher-Ricci flow (1.1) the scalar curvature $R$ is uniformly bounded on any compact subset of $X_{\text {reg. }}$. More precisely, there exist constants $\lambda, C>0$ such that

$$
-C \leq R \leq \frac{C}{|S|_{h}^{2 \lambda}} .
$$

Proof. It suffices to give an upper bound for $R$ since the scalar curvature $R$ is uniformly bounded from below by the maximum principle (cf. SSoTi]). Notice that $R_{i \bar{j}}=-u_{i \bar{j}}-\chi_{i \bar{j}}$ and then

$$
R=-\Delta u-\operatorname{tr}_{\omega}(\chi)
$$

By Corollary 5.4 and the partial second-order estimate, there exist constants $\lambda_{6}$, $C>0$ such that

$$
R \leq \frac{C}{|S|_{h}^{2 \lambda}}
$$




\section{Step 4. Uniform convergence.}

Let $\varphi_{\infty}$ be the unique solution solving equation (3.22) in Theorem 3.2. We identify $f^{*} \varphi_{\infty}$ and $\varphi_{\infty}$ for convenience.

Since $K_{X}$ is semi-ample, there exists an ample line bundle $L$ on $X_{\text {can }}$ such that $K_{X}=f^{*} L=\frac{1}{m} f^{*} \mathcal{O}(1)$ for a fixed pluricanonical map. Let $D$ be an ample divisor on $X_{\text {can }}$ such that $[D]=\mu[L]$ for a sufficiently large integer $\mu, X_{\text {can }} \backslash X_{\text {can }}^{\circ} \subset$ $D$ and $\varphi_{\infty} \in C^{\infty}\left(X_{c a n} \backslash D\right)$. Let $S_{D}$ be the defining section of $D$. Let $h_{F S}$ be the Fubini study metric on $\mathcal{O}(1)$ induced by the pluricanonical map. Then there exists a continuous Hermitian metric $h_{D}=\left(h_{F S}\right)^{\frac{\mu}{m}} e^{-\mu \varphi_{\infty}}$ on $L^{\mu}$ such that $-\sqrt{-1} \partial \bar{\partial} \log h_{D}=\mu \chi_{\infty}$ since $\varphi_{\infty}$ is continuous.

We define

$$
B_{r}(D)=\left\{y \in X_{c a n} \mid \operatorname{dist}_{\chi}(y, D) \leq r\right\}
$$

to be the geodesic tubular neighborhood of $D$ with respect to $\chi$ and we let $\mathcal{B}_{r}(D)=$ $f^{-1}\left(B_{r}(D)\right)$.

Since $\varphi_{\infty}$ is bounded on $X, \varphi$ is uniformly bounded from above. Therefore for any $\epsilon>0$, there exists $r_{\epsilon}>0$ with $\lim _{\epsilon \rightarrow 0} r_{\epsilon}=0$, such that for any $z \in \mathcal{B}_{r_{\epsilon}}(D)$ and $t \geq 0$ we have

$$
\left(\varphi-\varphi_{\infty}+\epsilon \log \left|S_{D}\right|_{h_{D}}^{2}\right)(t, z)<-1
$$

and

$$
\left(\varphi-\varphi_{\infty}-\epsilon \log \left|S_{D}\right|_{h_{D}}^{2}\right)(t, z)>1
$$

Let $\eta_{\epsilon}$ be a smooth cutoff function on $X_{\text {can }}$ such that $\eta_{\epsilon}=1$ on $X_{c a m} \backslash B_{r_{\epsilon}}(D)$ and $\eta_{\epsilon}=0$ on $B_{\frac{r_{\epsilon}}{2}}(D)$.

Consider the semi-flat closed $(1,1)$-form $\omega_{S F}$ introduced in Lemma 3.2. Suppose it is given by $\omega_{S F}=\omega_{0}+\sqrt{-1} \partial \bar{\partial} \rho_{S F}$ and $\rho_{S F}$ blows up near the singular fibres. We let $\rho_{\epsilon}$ be an approximation for $\rho_{S F}$ given by

$$
\rho_{\epsilon}=\left(f^{*} \eta_{\epsilon}\right) \rho_{S F} .
$$

We also define $\omega_{S F, \epsilon}=\omega_{0}+\sqrt{-1} \partial \bar{\partial} \rho_{\epsilon}$. Now we define the twisted difference of $\varphi$ and $\varphi_{\infty}$ by

$$
\psi_{\epsilon}^{-}=\varphi-(1+\epsilon) \varphi_{\infty}-e^{-t} \rho_{\epsilon}+\epsilon \log \left|S_{D}\right|_{h_{D}}^{2}
$$

and

$$
\psi_{\epsilon}^{+}=\varphi-(1-\epsilon) \varphi_{\infty}-e^{-t} \rho_{\epsilon}-\epsilon \log \left|S_{D}\right|_{h_{D}}^{2},
$$

where $S_{D}$ is the defining section of $D$ and $h$ is a fixed smooth Hermitian metric of the line bundle induced by $[D]$. We identify $f^{*}\left(\left|S_{D}\right|_{h_{D}}^{2}\right)$ and $\left|S_{D}\right|_{h_{D}}^{2}$ for convenience.

Proposition 5.3. There exists $\epsilon_{0}>0$ such that for any $0<\epsilon<\epsilon_{0}$, there exists $T_{\epsilon}>0$ such that for any $z \in X$ and $t>T_{\epsilon}$ we have

$$
\psi_{\epsilon}^{-}(t, z) \leq 2 \epsilon
$$

and

$$
\psi_{\epsilon}^{+}(t, z) \geq-2 \epsilon
$$


Proof. The evolution equation for $\psi_{\epsilon}^{-}$is given by

$$
\begin{aligned}
\frac{\partial \psi_{\epsilon}^{-}}{\partial t}= & \log \frac{e^{(n-\kappa) t}\left(\left(1+\epsilon \mu-e^{-t}\right) \chi_{\infty}+e^{-t} \omega_{S F, \epsilon}+\sqrt{-1} \partial \bar{\partial} \psi_{\epsilon}^{-}\right)^{n}}{C_{n, \kappa} \chi_{\infty}^{\kappa} \wedge \omega_{S F}^{n-\kappa}} \\
& -\psi_{\epsilon}^{-}-\epsilon \varphi_{\infty}+\epsilon \log |S|_{h}^{2} .
\end{aligned}
$$

Since $\rho_{\epsilon}$ is bounded on $X$, we can always choose $T_{1}>0$ sufficiently large such that for $t>T_{1}$,

(1) $\psi_{\epsilon}^{-}(t, z)<-\frac{1}{2}$ on $\mathcal{B}_{r_{\epsilon}}(D)$,

(2) $\sum_{p=0}^{\kappa-1} C_{n, p} e^{-(n-p) t}\left|\frac{\chi_{\infty}^{p} \wedge \omega_{S F}^{n-p}}{\chi_{\infty}^{\kappa} \wedge \omega_{S F}^{n-\kappa}}\right| \leq \epsilon$ on $X \backslash \mathcal{B}_{r_{\epsilon}}(D)$.

We will discuss in two cases for $t>T_{1}$.

(1) If $\psi_{\epsilon, \text { max }}^{-}(t)=\max _{X} \psi_{\epsilon}^{-}(t, \cdot)=\psi_{\epsilon}^{-}\left(t, z_{\max , t}\right)>0$ for all $t>T_{1}$, then $z_{\max , t} \in X \backslash \mathcal{B}_{r_{\epsilon}}(D)$ for all $t>T_{1}$ and so $\omega_{S F, \epsilon}\left(z_{\max , t}\right)=\omega_{S F}\left(z_{\max , t}\right)$. Applying the maximum principle at $z_{\max , t}$, we have

$$
\begin{aligned}
& \frac{\partial \psi_{\epsilon}^{-}}{\partial t}\left(t, z_{\max , t}\right) \\
& \leq\left(\log \frac{e^{(n-\kappa) t}\left(\left(1+\epsilon \mu-e^{-t}\right) \chi_{\infty}-\epsilon \varphi_{\infty}+e^{-t} \omega_{S F, \epsilon}\right)^{n}}{C_{n, \kappa} \chi_{\infty}^{\kappa} \wedge \omega_{S F}^{n-\kappa}}\right. \\
& \left.-\psi_{\epsilon}^{-}-\epsilon \varphi_{\infty}+\epsilon \log |S|_{h}^{2}\right)\left(t, z_{\max , t}\right) \\
& =\left(\log \frac{\sum_{p=0}^{\kappa}\left(\begin{array}{c}
n \\
\kappa
\end{array}\right)\left(1+\epsilon \mu-e^{-t}\right)^{p} \chi_{\infty}^{p} \wedge \omega_{S F, \epsilon}^{n-p}}{\left(\begin{array}{c}
n \\
\kappa
\end{array}\right) \chi_{\infty}^{\kappa} \wedge \omega_{S F}^{n-\kappa}}\right. \\
& \left.-\psi_{\epsilon}^{-}-\epsilon \varphi_{\infty}+\epsilon \log |S|_{h}^{2}\right)\left(t, z_{\max , t}\right) \\
& \leq-\psi_{\epsilon}^{-}\left(t, z_{\max , t}\right)+\log (1+(\mathcal{A}+1) \epsilon)+\epsilon .
\end{aligned}
$$

Applying the maximum principle again, we have

$$
\psi_{\epsilon}^{-} \leq(\mathcal{A}+2) \epsilon+O\left(e^{-t}\right) \leq(\mathcal{A}+3) \epsilon
$$

if we choose $\epsilon$ sufficiently small in the beginning and then $t$ sufficiently large.

(2) If there exists $t_{0} \geq T_{1}$ such that $\max _{z \in X} \psi_{\epsilon}^{-}\left(t_{0}, z\right)=\psi_{\epsilon}^{-}\left(t_{0}, z_{0}\right)<0$ for some $z_{0} \in X$, then assume $t_{1}$ is the first time when $\max _{z \in X, t \leq t_{1}} \psi_{\epsilon}^{-}(t, z)=$ $\psi_{\epsilon}^{-}\left(t_{1}, z_{1}\right) \geq(\mathcal{A}+3) \epsilon$. Then $z_{1} \in X \backslash B_{r_{\epsilon}}(D)$ and applying the maximum 
principle we have

$$
\begin{aligned}
& \psi_{\epsilon}^{-}\left(t_{1}, z_{1}\right) \\
& \leq\left(\log \frac{e^{(n-\kappa) t}\left(\left(1+\epsilon \mu-e^{-t}\right) \chi_{\infty}+e^{-t} \omega_{S F, \epsilon}\right)^{n}}{\left(\begin{array}{c}
n \\
\kappa
\end{array}\right) \chi_{\infty}^{\kappa} \wedge \omega_{S F}^{n-\kappa}}\right. \\
& \left.-\psi_{\epsilon}^{-}-\epsilon \varphi_{\infty}+\epsilon \log |S|_{h}^{2}\right)\left(t_{1}, z_{1}\right) \\
& \leq \log (1+(\mathcal{A}+1) \epsilon)+\epsilon<(\mathcal{A}+2) \epsilon,
\end{aligned}
$$

which contradicts the assumption that $\psi_{\epsilon}^{-}\left(t_{1}, z_{1}\right) \geq(\mathcal{A}+3) \epsilon$. Hence we have

$$
\psi_{\epsilon}^{-} \leq(\mathcal{A}+3) \epsilon
$$

By the same argument we have

$$
\psi_{\epsilon}^{+} \geq-(\mathcal{A}+3) \epsilon
$$

This completes the proof.

Proposition 5.4. On any compact set $K$ of $X \backslash D$, we have

$$
\lim _{t \rightarrow \infty}\left\|\varphi(t, \cdot)-\varphi_{\infty}(\cdot)\right\|_{C^{0}(K)}=0 .
$$

Proof. By Proposition 5.3, we have for $t>T_{\epsilon}$,

$$
\varphi_{\infty}(t, z)+\epsilon \log |S|_{h}^{2}(t, z)-3 \epsilon \leq \varphi(t, z) \leq \varphi_{\infty}(t, z)-\epsilon \log |S|_{h}^{2}(t, z)+3 \epsilon .
$$

Then the proposition is proved by letting $\epsilon \rightarrow 0$.

5.3. Kähler-Ricci flow and minimal model program. The Kähler-Ricci flow on projective manifolds of positive Kodaira dimension seems to be closely related to the minimal model program in algebraic geometry.

For any nonsingular minimal model $X$ of positive Kodaira dimension, the canonical line bundle $K_{X}$ is nef and so the Kähler-Ricci flow (5.60) has long time existence [TiZha]. The abundance conjecture predicts that $K_{X}$ is semi-ample; hence the canonical ring of $X$ is finitely generated. If we assume the abundance conjecture, the Kähler-Ricci flow will converge to the unique canonical metric on the canonical model $X_{\text {can }}$ associated to $X$ for any initial Kähler metric by Theorem A.

If $X$ is not minimal, the Kähler-Ricci flow (5.60) will develop finite time singularities. Let $T_{1}$ be the first time such that $e^{-t}\left[\omega_{0}\right]-\left(1-e^{-t}\right) 2 \pi c_{1}(X)$ fails to be a Kähler class. Adopting arguments in [TiZha], one can show that there is a unique limiting current $\omega_{T_{1}}(\cdot)=\lim _{t \rightarrow T_{1}^{-}} \omega(t, \cdot) \in e^{-T_{1}}\left[\omega_{0}\right]-\left(1-e^{-T_{1}}\right) 2 \pi c_{1}(X)$ and it is smooth outside an analytic subvariety of $X$. Furthermore, the local potential $\varphi_{T_{1}}$ of $\omega_{T_{1}}$ is continuous. We conjecture that $X_{1}$, the metric completion of $\omega_{T_{1}}$, is again a projective variety and $X_{1}$ can be obtained by a certain standard algebraic procedure such as a blow-down or flip. It is reasonable to expect that such a variety $X_{1}$ does not have too bad singularities. In particular, we expect that a weak Kähler-Ricci flow can be defined on $X_{1}$. Supposing that this is true, we hope 
that the above procedure can be repeated as long as the canonical line bundle is not nef. We further conjecture that after repeating the above process finitely many times, we obtain the metric completions $X_{1}, X_{2}, \ldots, X_{N}$ such that $K_{X_{N}}$ is nef ! Consequently, $X_{N}$ is a minimal model of $X$.

It provides a new understanding of the minimal model program from an analytic point of view. We believe that it is interesting to further explore this connection between the minimal model program and the study of the regularity and convergence problem of the Kähler-Ricci flow on projective varieties.

\section{Adjunction formulas for Energy functionals}

6.1. Generalized constant scalar curvature Kähler metrics. In fact, the canonical metrics in Section 3 belong to a class of Kähler metrics which generalize Calabi's extremal metrics. Let $Y$ be a Kähler manifold of complex dimension $n$ together with a fixed closed $(1,1)$-form $\theta$. Fix a Kähler class $[\omega]$, denote by $\mathcal{K}_{[\omega]}$ the space of Kähler metrics within the same Kähler class, that is, all Kähler metrics of the form $\omega_{\varphi}=\omega+\sqrt{-1} \partial \bar{\partial} \varphi$. One may consider the following equation:

$$
\bar{\partial} V_{\varphi}=0,
$$

where $V_{\varphi}$ is defined by

$$
\omega_{\varphi}\left(V_{\varphi}, \cdot\right)=\bar{\partial}\left(S\left(\omega_{\varphi}\right)-\operatorname{tr}_{\omega_{\varphi}}(\theta)\right) .
$$

Clearly, when $\theta=0$, (6.80) is exactly the equation for Calabi's extremal metrics. For this reason, we call a solution of (6.80) a generalized extremal metric. If $Y$ does not admit any nontrivial holomorphic vector fields, then any generalized extremal metric $\omega_{\varphi}$ satisfies

$$
S\left(\omega_{\varphi}\right)-\operatorname{tr}_{\omega_{\varphi}}(\theta)=\mu,
$$

where $\mu$ is the constant given by

$$
\mu=\frac{n\left(2 \pi c_{1}(Y)-[\theta]\right) \cdot[\omega]^{n-1}}{[\omega]^{n}} .
$$

Moreover, if $2 \pi c_{1}(Y)-[\theta]=\lambda[\omega]$, then any such a metric satisfies

$$
\operatorname{Ric}\left(\omega_{\varphi}\right)=\lambda \omega_{\varphi}+\theta
$$

that is, $\omega_{\varphi}$ is a generalized Kähler-Einstein metric. This can be proved by an easy application of the Hodge theory. More interestingly, if we take $\theta$ to be the pull-back of $\omega_{W P}$ by $f: X_{c a n}^{\circ} \rightarrow \mathcal{M}_{C Y}$, then we get back those generalized Kähler-Einstein metrics which arise from limits of the Kähler-Ricci flow.

Let $f: X \rightarrow \Sigma$ be a Kähler surface admitting a nonsingular holomorphic fibration over a Riemann surface $\Sigma$ of genus greater than one, with fibres of genus at least 2. Let $V$ be the vertical tangent bundle of $X$ and $\left[\omega_{t}\right]=-f^{*} c_{1}(\Sigma)-t c_{1}(V)$.

Let $\chi$ be a Kähler form in $-c_{1}(\Sigma)$ and $\omega_{0} \in-c_{1}(V)$. Then $\omega_{0}=\omega_{H} \oplus \theta \chi$, where $\omega_{H}$ is the hyperbolic Kähler form on each fiber and $\theta$ is a smooth function on $X$. We then set

$$
\omega_{t}=\chi+t \omega_{0}
$$

The following theorem is proved by Fine in [Fi]. 
Theorem 6.1. For $t>0$ sufficiently small, there exists a constant scalar curvature Kähler metric in $\left[\omega_{t}\right]$. Furthermore, such a family of constant scalar curvature Kähler metrics converges to a Kähler metric $\chi_{\infty}$ on $\Sigma$ defined by

$$
S\left(\chi_{\infty}\right)-\operatorname{tr}_{\chi_{\infty}}(\theta)=\text { const }
$$

where $\theta$ is the Weil-Petersson metric pulled back from the moduli spaces of the fibre curves.

\subsection{Asymptotics of the Mabuchi energy by the large Kähler structure}

limits. Let $X$ be an $n$-dimensional compact Kähler manifold and $\omega$ a Kähler form. The Mabuchi energy functional $\mathcal{K}_{\omega}(\cdot)$ is defined on $P S H(X, \omega)$ as follows:

$$
\mathcal{K}_{\omega}(\varphi)=\int_{X} \log \frac{\omega_{\varphi}^{n}}{\omega^{n}} \omega_{\varphi}^{n}-\sum_{j=0}^{n-1} \int_{X} \varphi \operatorname{Ric}(\omega) \wedge \omega^{j} \wedge \omega_{\varphi}^{n-j-1}+\frac{n \mu}{n+1} \sum_{j=0}^{n} \int_{X} \varphi \omega^{j} \wedge \omega_{\varphi}^{n-j}
$$

where $\omega_{\varphi}=\omega+\sqrt{-1} \partial \bar{\partial} \varphi$ and $\mu=\frac{2 n \pi c_{1}(X) \cdot[\omega]^{n-1}}{[\omega]^{n}}$.

Definition 6.1. Let $X$ be a compact Kähler manifold of complex dimension $n$. Let $\omega$ be a Kähler metric and $\theta$ a closed $(1,1)$-form on $X$. Then the generalized Mabuchi energy functional $\mathcal{K}_{\omega, \theta}(\cdot)$ is defined by

$\mathcal{K}_{\omega, \theta}(\varphi)=\int_{X} \log \frac{\omega_{\varphi}^{n}}{\omega^{n}} \omega_{\varphi}^{n}-\sum_{j=0}^{n-1} \int_{X} \varphi(\operatorname{Ric}(\omega)-\theta) \wedge \omega^{j} \wedge \omega_{\varphi}^{n-j-1}+\frac{n \mu}{n+1} \sum_{j=0}^{n} \int_{X} \varphi \omega^{j} \wedge \omega_{\varphi}^{n-j}$, where $\mu=\frac{\left(2 \pi c_{1}(X)-[\theta]\right) \cdot[\omega]^{n-1}}{[\omega]^{n}}$.

The following proposition can be proved by a straightforward calculation.

\section{Proposition 6.1.}

$$
\delta \mathcal{K}_{\omega, \theta}=-\int_{X} \delta \varphi\left(S\left(\omega_{\varphi}\right)-\operatorname{tr}_{\omega_{\varphi}}(\theta)-\mu\right) \omega_{\varphi}^{n}
$$

Therefore

$$
\mathcal{K}_{\omega, \theta}(\varphi)=-\int_{0}^{1} \int_{X} \dot{\varphi}_{t}\left(S\left(\omega_{t}\right)-\operatorname{tr}_{\omega_{t}}(\theta)-\mu\right) \omega_{t}^{n} d t
$$

where $\left\{\varphi_{t}\right\}_{t \in[0,1]}$ is a smooth path in $\operatorname{PSH}(X, \omega)$ with $\varphi_{0}=0$ and $\varphi_{1}=\varphi$, and $\omega_{t}=\omega+\sqrt{-1} \partial \bar{\partial} \varphi_{t}$. The formula (6.87) is independent of the choice of the path $\varphi_{t}$.

Let $X$ be an $n$-dimensional compact Kähler manifold with semi-ample canonical line bundle. Suppose $0<\operatorname{kod}(X)=\kappa<n$ and $X_{\text {can }}=X_{c a n}^{\circ}$, i.e., $X_{c a n}$ is nonsingular and the algebraic fibration $f: X \rightarrow X_{c a n}$ has no singular fibre.

Fix $\chi \in-2 \pi c_{1}(X)$ as in Section 3.1 and let $\omega_{0}$ be an arbitrary Kähler form with $\int_{X_{s}} \omega_{0, s}^{n-\kappa}=1$, where $X_{s}=f^{-1}(s)$ and $\omega_{0, s}=\left.\omega_{0}\right|_{X_{s}}$. Let $\omega_{t}=\chi+t \omega_{0}$ and $\omega_{\varphi}=\omega_{t}+\sqrt{-1} \partial \bar{\partial} \varphi$. Let $\omega_{S F}$ be the semi-flat form in $\left[\omega_{0}\right]$. Then $\varphi$ can always be decomposed as

$$
\varphi=\bar{\varphi}+t \psi
$$

where $\bar{\varphi}=\int_{X_{y}} \varphi \omega_{S F}^{n-k}$ is the pushforward of $\varphi$ with respect to the Ricci-flat Kähler metric on the fibres. 
Theorem 6.2. Along the above class deformation of the Kähler class on X,

$$
\mathcal{K}_{\omega_{t}}(\varphi)=\left(\begin{array}{c}
n \\
\kappa
\end{array}\right) t^{n-\kappa}\left(\mathcal{K}_{\chi, \omega_{W} P}(\bar{\varphi})+\mathcal{L}_{\chi, \chi_{\bar{\varphi}}, \omega_{0}}(\psi)\right)+O\left(t^{n-\kappa+1}\right)
$$

and

$$
\begin{aligned}
\mathcal{L}_{\chi, \chi_{\bar{\varphi}}, \omega_{0}}(\psi)= & \int_{s \in X_{c a n}}\left(\int_{X_{s}} \log \frac{\omega_{\psi, s}^{n-\kappa}}{\left.\omega_{0, s}^{n-\kappa} \omega_{\psi, s}^{n-\kappa}\right) \chi_{\bar{\varphi}}^{\kappa}}\right. \\
& -\sum_{j=0}^{n-\kappa-1} \sum_{i=0}^{\kappa} A_{i, j} \int_{s \in X_{c a n}}\left(\int_{X_{s}} \psi \operatorname{Ric}\left(\omega_{0, s}\right)\right. \\
& \left.\wedge \omega_{0, s}^{j} \wedge \omega_{\psi, s}^{n-\kappa-1-j}\right) \chi^{i} \wedge \chi_{\bar{\varphi}}^{\kappa-i},
\end{aligned}
$$

where $A_{i, j}=\left(\begin{array}{c}n \\ \kappa\end{array}\right)^{-1}\left(\begin{array}{c}i+j \\ i\end{array}\right)\left(\begin{array}{c}n-1-i-j \\ \kappa-i\end{array}\right)$ and $\mathcal{K}_{\chi, \omega_{W P}}(\cdot)$ is the generalized Mabuchi energy on $X_{\text {can }}$.

In particular, when $\chi=\chi_{\bar{\varphi}}$,

$$
\mathcal{L}_{\chi, \chi_{\bar{\varphi}}, \omega_{0}}(\psi)=\int_{s \in X_{c a n}} \mathcal{K}_{\omega_{0, s}}(\psi) \chi^{\kappa}
$$

where $\mathcal{K}_{\omega_{0, s}}(\cdot)$ is the Mabuchi energy on the Calabi-Yau fibre $X_{s}$.

Proof. The proof boils down to a direct computation. First, calculate (6.89)

$$
\int_{X} \log \frac{\omega_{\varphi}^{n}}{\omega^{n}} \omega_{\varphi}^{n}=t^{n-k}\left(\begin{array}{c}
n \\
\kappa
\end{array}\right)\left(\int_{X} \log \frac{\chi_{\bar{\varphi}}^{\kappa}}{\chi^{\kappa}} \chi_{\bar{\varphi}}^{\kappa}+\int_{X_{c a n}}\left(\int_{X_{y}} \log \frac{\omega_{\psi}^{n-\kappa}}{\omega^{n-\kappa}} \omega_{\psi}^{n-\kappa}\right) \chi_{\bar{\varphi}}^{\kappa}+O(t)\right) .
$$

Also

$$
\begin{aligned}
& -\sum_{j=0}^{n-1} \int_{X} \varphi \operatorname{Ric}(\omega) \wedge \omega^{j} \wedge \omega_{\varphi}^{n-j-1} \\
& =t^{n-\kappa} \sum_{j=1}^{\kappa-1}\left(\begin{array}{c}
n \\
\kappa
\end{array}\right) \int_{X_{c a n}} \bar{\varphi}\left(-\operatorname{Ric}(\chi)+\omega_{W P}\right) \wedge \chi^{j} \wedge \chi_{\bar{\varphi}}^{\kappa-j-1} \\
& \quad-t^{n-\kappa} \sum_{j=0}^{n-\kappa-1} \sum_{i=j}^{\kappa+j} A_{i, j} \int_{s \in X_{c a n}}\left(\int_{X_{s}} \operatorname{Ric}\left(\omega_{0, s}\right) \wedge \omega_{0, s}^{j} \wedge \omega_{\psi, s}^{n-\kappa-1-j}\right) \chi^{i} \wedge \chi_{\bar{\varphi}}^{\kappa-i} \\
& \quad+O\left(t^{n-\kappa+1}\right)
\end{aligned}
$$

and

(6.90) $\sum_{j=0}^{n} \int_{X} \varphi \omega_{t}^{j} \wedge \omega_{\varphi}^{n-j}=\left(\begin{array}{c}n+1 \\ \kappa+1\end{array}\right) t^{n-\kappa} \sum_{j=0}^{\kappa} \int_{X_{c a n}} \bar{\varphi} \chi^{j} \wedge \chi_{\bar{\varphi}}^{\kappa-j}+O\left(t^{n-\kappa+1}\right)$. 
The theorem follows from a straightforward calculation by combining the above formulas:

$$
\begin{aligned}
\mathcal{K}_{\omega_{t}}(\varphi) & \\
= & \left(\begin{array}{c}
n \\
\kappa
\end{array}\right) t^{n-\kappa}\left(\int_{X_{c a n}} \log \frac{\chi_{\bar{\varphi}}^{\kappa}}{\chi^{\kappa}} \chi_{\bar{\varphi}}^{\kappa}-\int_{X_{c a n}} \bar{\varphi}\left(\operatorname{Ric}(\chi)-\omega_{W P}\right) \wedge \chi^{j} \wedge \chi_{\bar{\varphi}}^{\kappa-j}\right. \\
& +\left(\begin{array}{c}
n \\
\kappa
\end{array}\right) t^{n-\kappa} \int_{s \in X_{c a n}}\left(\int_{X_{s}} \log \frac{\omega_{\psi, s}^{n-\kappa}}{\left.\omega_{0, s}^{n-\kappa} \omega_{\psi, s}^{n-\kappa}\right) \chi_{\bar{\varphi}}^{\kappa}} \wedge \chi_{\bar{\varphi}}^{\kappa-j}\right) \\
& -t^{n-\kappa} \sum_{j=0}^{n-\kappa-1} \sum_{i=j}^{m+j} \int_{s \in X_{c a n}}\left(\int_{X_{s}} \operatorname{Ric}\left(\omega_{0, s}\right) \wedge \omega_{0, s}^{j} \wedge \omega_{\psi, s}^{n-\kappa-1-j}\right) \chi^{i-j} \wedge \chi_{\bar{\varphi}}^{m-i+j} \\
& +O\left(t^{n-\kappa+1}\right),
\end{aligned}
$$

where $\bar{\mu}=\frac{\left(2 \pi c_{1}\left(X_{c a n}\right)-\left[\omega_{W P}\right]\right) \cdot[\chi]^{\kappa-1}}{[\chi]^{\kappa}}$.

We also investigate the asymptotic properties of the Mabuchi energy in the case of a fibred space studied by Fine in [Fi].

Let $f: X \rightarrow \Sigma$ be a Kähler surface admitting a nonsingular holomorphic fibration over $\Sigma$, with fibres of genus at least 2 . We also assume $c_{1}(\Sigma)<0$. Let $V$ be the vertical tangent bundle of $X$ and $\left[\omega_{t}\right]=-f^{*} c_{1}(\Sigma)-t c_{1}(V)$.

Let $\chi, \omega_{0} \in-c_{1}(V)$ and $\omega_{t}$ be defined as in Section 6.1. We consider the asymptotic properties of the Mabuchi energy $\mathcal{K}_{\omega_{t}}(\cdot)$ as $t$ tends to 0.

Theorem 6.3. Let $\omega_{0} \in-c_{1}(V)$ be a closed $(1,1)$-form such that its restriction on each fibre is a hyperbolic metric. Let $\omega_{t}=\chi+t \omega_{0}$ and $\omega_{\varphi}=\omega_{t}+\sqrt{-1} \partial \bar{\partial} \varphi$ be a metric deformation, where $\varphi \in C^{\infty}(\Sigma)$. Then we have

$$
\mathcal{K}_{\omega_{t}}(\varphi)=2 t \mathcal{K}_{\chi, \theta}(\varphi)+O\left(t^{2}\right) .
$$

Theorem 6.2 and Theorem 6.3 can be considered as an adjunction type formula for the Mabuchi energy on an algebraic fibre space.

\section{ACKNOWLEDGMENTS}

The first author would like to thank H. Fang, D.H. Phong, J. Sturm, V. Tosatti, B. Weinkove and Z. Zhou for enlightening discussions. Both authors thank MSRI for its hospitality during the program on geometric evolution equations and related topics where part of the work was carried out. The second author would also like to thank the Clay Mathematics Institute for financial support during his visit to MSRI.

\section{REFERENCES}

[Au] Aubin, T. Equations du type Monge-Ampère sur les variétés Kähleriennes compacts, Bull. Sc. Math. 102 (1976), 119-121. MR0494932 (81d:53047)

[BaMu] Bando, S. and Mabuchi, T., Uniqueness of Einstein Kähler metrics modulo connected group actions, Algebraic geometry, Sendai, 1985, 11-40, Adv. Stud. Pure Math., 10, North-Holland, Amsterdam, 1987. MR946233 (89c:53029)

[BiCaHaMc] Birkar, C., Cascini, P., Hacon, C. and McKernan, J., Existence of minimal models for varieties of log general type, J. Amer. Math. Soc. 23 (2010), no. 2, 405-468. MR2601039 (2011f:14023) 
[Ca] Cao, H., Deformation of Kähler metrics to Kähler-Einstein metrics on compact Kähler manifolds, Invent. Math. 81 (1985), no. 2, 359-372. MR799272 (87d:58051)

[Ch] Chow, B., The Ricci flow on the 2-sphere, J. Differential Geom. 33 (1991), no. 2, 325-334. MR 1094458 (92d:53036)

[ChTi] Chen, X.X. and Tian, G., Ricci flow on Kähler-Einstein surfaces, Invent. Math. 147 (2002), no. 3, 487-544. MR1893004 (2003c:53095)

[ChYa] Cheng, S. Y. and Yau, S. T., Differential equations on Riemannian manifolds and their geometric applications, Comm. Pure Appl. Math. 28 (1975), no. 3, 333-354. MR0385749 (52:6608)

[ClKoMo] Clemens, H., Kollar, J. and Mori, S., Higher-dimensional complex geometry, Astérisque No. 166 (1988), 144 pp. (1989). MR.1004926 (90j:14046)

[DePa] Demailly, J-P. and Pali, N., Degenerate complex Monge-Ampère equations over compact Kähler manifolds, Internat. J. Math. 21 (2010), no. 3, 357-405. MR2647006

[DiZh] Dinew, S. and Zhang, Z., Stability of Bounded Solutions for Degenerate Complex Monge-Ampère equations, Adv. Math. 225 (2010), no. 1, 367-388. MR2669357

[Do] Donaldson, S. K., Scalar curvature and projective embeddings, I., J. Differential Geom. 59 (2001), no. 3, 479-522. MR.1916953 (2003j:32030)

[EyGuZe1] Eyssidieux, P., Guedj, V. and Zeriahi, A., Singular Kähler-Einstein metrics, J. Amer. Math. Soc. 22 (2009), no. 3, 607-639. MR2505296(2010k:32031)

[EyGuZe2] Eyssidieux, P., Guedj, V. and Zeriahi, A., A priori $L^{\infty}$-estimates for degenerate complex Monge-Ampère equations, Int. Math. Res. Not. IMRN 2008, Art. ID rnn 070, 8 pp. MR2439574 (2009f:32055)

[FaLu] Fang, H. and Lu, Z., Generalized Hodge metrics and BCOV torsion on Calabi-Yau moduli, J. Reine Angew. Math. 588 (2005), 49-69. MR2196728(2007a:32028)

[Fi] Fine, J., Constant scalar curvature Kähler metrics on fibred complex surfaces, J. Differential Geom. 68 (2004), no. 3, 397-432. MR2144537 (2005m:32045)

[GrWi] Gross, M. and Wilson, P. M. H., Large complex structure limits of K3 surfaces, J. Differential Geom. 55 (2000), no. 3, 475-546. MR.1863732 (2003a:32042)

[Ha] Hamilton, R., Three-manifolds with positive Ricci curvature, J. Differential Geom. 17 (1982), no. 2, 255-306. MR664497 (84a:53050)

[Kol1] Kolodziej, S., The complex Monge-Ampère equation, Acta Math. 180 (1998), no. 1, 69-117. MR:1618325 (99h:32017)

[Kol2] Kolodziej, S., The Monge-Ampère equation on compact Kähler manifolds, Indiana Univ. Math. J. 52 (2003), no. 3, 667-686. MR1986892(2004i:32062)

[LiYa] Li, P. and Yau, S.T., Estimates of eigenvalues of a compact Riemannian manifold, Geometry of the Laplace operator (Proc. Sympos. Pure Math., Univ. Hawaii, Honolulu, Hawaii, 1979), pp. 205-239, Proc. Sympos. Pure Math., XXXVI, Amer. Math. Soc., Providence, R.I., 1980. MR573435 (81i:58050)

[La] Lazarsfeld, J., Positivity in algebraic geometry. I. Classical setting: line bundles and linear series, A Series of Modern Surveys in Mathematics, 48. Springer-Verlag, Berlin, 2004. xviii+387 pp. MR2095471(2005k:14001a)

[Lo] Lott, J., On the long-time behavior of type-III Ricci flow solutions, Math. Ann. 339 (2007), no. 3, 627-666. MR2336062 (2008i:53093)

[Pe] Perelman, P., The entropy formula for the Ricci flow and its geometric applications, preprint math.DG/0211159.

[PhSt] Phong, D. H. and Sturm, J., On stability and the convergence of the Kähler-Ricci flow, J. Differential Geom. 72 (2006), no. 1, 149-168. MR2215459 (2007a:53128)

[Si1] Siu, Y-T., Invariance of plurigenera, Invent. Math. 134 (1998), no. 3, 661-673. MR.1660941 (99i:32035)

[Si2] Siu, Y-T., Multiplier ideal sheaves in complex and algebraic geometry, Sci. China Ser. A 48 (2005), suppl., 1-31. MR2156488 (2007a:32023)

[Si3] Siu, Y-T., A General Non-Vanishing Theorem and an Analytic Proof of the Finite Generation of the Canonical Ring, arXiv:math/0610740.

[SoTi] Song, J. and Tian, G., The Kähler-Ricci flow on minimal surfaces of positive Kodaira dimension, Invent. Math. 170 (2007), no. 3, 609-653. MR2357504 (2008m:32044) 
[SoWe] Song, J. and Weinkove, B., On the convergence and singularities of the J-flow with applications to the Mabuchi energy, Comm. Pure Appl. Math. 61 (2008), 210-229. MR2368374 (2009a:32038)

[StYaZa] Strominger, A., Yau, S.T. and Zaslow, E., Mirror symmetry is T-duality, Nuclear Phys. B 479 (1996), no. 1-2, 243-259. MR1429831 (97j:32022)

[Ti1] Tian, G., On Kähler-Einstein metrics on certain Kähler manifolds with $C_{1}(M)>0$, Invent. Math. 89 (1987), no. 2, 225-246. MR894378 (88e:53069)

[Ti2] Tian, G., On Calabi's conjecture for complex surfaces with positive first Chern class, Invent. Math. 101, no. 1 (1990), 101-172. MR.1055713 (91d:32042)

[Ti3] Tian, G., On a set of polarized Kähler metrics on algebraic manifolds, J. Differential Geom. 32 (1990), no. 1, 99-130. MR.1064867 (91j:32031)

[Ti4] Tian, G., Smoothness of the universal deformation space of compact Calabi-Yau manifolds and its Petersson-Weil metric, Mathematical aspects of string theory (San Diego, Calif., 1986), World Sci. Publishing, Singapore, 629-646. MR915841

[TiZha] Tian, G. and Zhang, Z., On the Kähler-Ricci flow on projective manifolds of general type, Chinese Ann. Math. Ser. B 27 (2006), no. 2, 179-192. MR2243679 (2007c:32029)

[TiZhu] Tian, G. and Zhu, X., Convergence of Kähler Ricci flow, J. Amer. Math. Soc. 20 (2007), no. 3, 675-699. MR2291916 (2007k:53107)

[To] Tosatti, V., Limits of Calabi-Yau metrics when the Kahler class degenerates, J. Eur. Math. Soc. (JEMS) 11 (2009), no. 4, 755-776. MR2538503 (2010j:32039)

[Ts1] Tsuji, H., Existence and degeneration of Kähler-Einstein metrics on minimal projective varieties of general type, Math. Ann. 281 (1988), 123-133. MR944606 (89e:53075)

[Ts2] Tsuji, H., Analytic Zariski decomposition, Proc. of Japan Acad. 61 (1992), 161-163. MR1193172 (93k:32014)

[Ts3] Tsuji, H., Generalized Bergmann Metrics and Invariance of Plurigenera, arXiv:math/960448.

[Ue] Ueno, K., Classification theory of projective varieties and compact complex spaces, notes written in collaboration with P. Cherenack, Lecture Notes in Mathematics, Vol. 439, Springer-Verlag, Berlin-New York, 1975. xix+278 pp. MR 0506253 (58:22062)

[Ya1] Yau, S.T., A general Schwarz lemma for Kähler manifolds, Amer. J. Math. 100 (1978), no. 1, 197-203. MR0486659(58:6370)

[Ya2] Yau, S.T. On the Ricci curvature of a compact Kähler manifold and the complex Monge-Ampère equation, I, Comm. Pure Appl. Math. 31 (1978), 339-411. MR 480350 (81d:53045)

[Zh] Zhang, Z., On degenerate Monge-Ampère equations over closed Kähler manifolds, Int. Math. Res. Not. 2006, Art. ID 63640, 18 pp. MR2233716 (2007b:32058)

Department of Mathematics, Rutgers University, Piscataway, New Jersey 08854

E-mail address: jiansong@math.rutgers.edu

School of Mathematical Sciences and BICMr, Peking University, Beijing, 100871, People's Republic of China and Department of Mathematics, Princeton University, Princeton, NeW Jersey 08544

E-mail address: tian@math.princeton.edu 